\title{
Chemo-enzymatic synthesis of tetra-, penta-, and hexasaccharide fragments of the capsular polysaccharide of Streptococcus pneumoniae type 14
}

\author{
John A.F. Joosten, Bart J. Lazet, Johannis P. Kamerling,* Johannes F.G. Vliegenthart \\ Bijvoet Center, Department of Bio-Organic Chemistry, Section of Glycoscience and Biocatalysis, Utrecht University, Padualaan 8, NL-3584 CH \\ Utrecht, The Netherlands
}

Received 16 April 2003; accepted 17 June 2003

\begin{abstract}
The chemo-enzymatic synthesis is described of $\beta$-D-Glc $p-(1 \rightarrow 6)-[\beta-\mathrm{D}-\mathrm{Gal} p-(1 \rightarrow 4)]-\beta-\mathrm{D}-\mathrm{Glc} p \mathrm{NAc}-(1 \rightarrow 3)-\beta$-D-Gal $p-(1 \rightarrow$ $\mathrm{O}\left(\mathrm{CH}_{2}\right)_{6} \mathrm{NH}_{2}(\mathbf{1}), \beta-\mathrm{D}-\mathrm{Glc} p-(1 \rightarrow 6)-[\beta-\mathrm{D}-\mathrm{Gal} p-(1 \rightarrow 4)]-\beta-\mathrm{D}-\mathrm{Glc} p \mathrm{NAc}-(1 \rightarrow 3)-\beta-\mathrm{D}-\mathrm{Gal} p-(1 \rightarrow 4)-\beta-\mathrm{D}-\mathrm{Glc} p-\left(1 \rightarrow \mathrm{O}(\mathrm{CH})_{6} \mathrm{NH} \mathrm{H}_{2}(2), \beta-\right.$ D-Gal $p-(1 \rightarrow 4)-\beta-\mathrm{D}-\mathrm{Glc} p$ NAc- $(1 \rightarrow 3)-\beta-\mathrm{D}-\mathrm{Gal} p-(1 \rightarrow 4)-\beta-\mathrm{D}-\mathrm{Glc} p-\left(1 \rightarrow \mathrm{O}\left(\mathrm{CH}_{2}\right)_{6} \mathrm{NH}_{2}(3)\right.$, and $\beta$-D-Gal $p-(1 \rightarrow 4)-\beta-\mathrm{D}-\mathrm{Gl} p$ NAc- $(1 \rightarrow$ 3)- $\beta$-D-Gal $p-(1 \rightarrow 4)-\beta-\mathrm{D}-\mathrm{Glc} p-(1 \rightarrow 6)-[\beta-\mathrm{D}-\mathrm{Gal} p-(1 \rightarrow 4)]-\beta-\mathrm{D}-\mathrm{Glc} p \mathrm{NAc}-\left(1 \rightarrow \mathrm{O}\left(\mathrm{CH}_{2}\right)_{6} \mathrm{NH}_{2}\right.$ (4), representing fragments of the repeating unit of the Streptococcus pneumoniae serotype 14 capsular polysaccharide. Linear intermediate oligosaccharides $\mathbf{5}-\mathbf{8}$ were synthesized via chemical synthesis, followed by enzymatic galactosylation using bovine milk $\beta$-1,4-galactosyltransferase as a catalyst. The title oligosaccharides form suitable compounds for conjugation with carrier proteins, to be tested as potential vaccines in animal models.
\end{abstract}

(C) 2003 Elsevier Ltd. All rights reserved.

Keywords: Carbohydrates; Streptococcus pneumoniae; Oligosaccharide synthesis

\section{Introduction}

The encapsulated bacterium Streptococcus pneumoniae is a major cause of life-threatening diseases such as otitis media, pneumonia, meningitis, bacteraemia, and septicaemia, ${ }^{1,2}$ mainly due to a growing resistance towards antibiotics. ${ }^{3,4}$ Vaccination with the available 23 -valent capsular polysaccharide (CPS) vaccines ${ }^{5}$ offers protection in healthy adults, but they are ineffective in the most important high-risk groups, such as infants, small children, immuno-compromised patients, and the elderly. ${ }^{6}$ Conjugation of $S$. pneumoniae carbohydrate antigens to a protein carrier results in a T-cell dependent neoglycoconjugate antigen that gives an efficient immune response in the high-risk groups, ${ }^{7}$ as have been shown for other bacteria., ${ }^{8,9}$ Currently, neoglycoconju-

\footnotetext{
* Corresponding author. Tel.: +31-30-2533479; fax: +3130-2540980.

E-mail address: j.p.kamerling@chem.uu.nl (J.P. Kamerling).
}

gate vaccines against $S$. pneumoniae serotypes have been introduced. ${ }^{7}$

The CPS of S. pneumoniae type 14 is built up from the tetrasaccharide repeating unit $\rightarrow 6)-[\beta-\mathrm{D}-\mathrm{Gal} p-(1 \rightarrow 4)]$ $\beta$-D-Glc $p$ NAc- $(1 \rightarrow 3)-\beta$-D-Gal $p$ - $(1 \rightarrow 4)-\beta$-D-Glc $p-(1 \rightarrow$ .$^{10}$ In earlier reports we have described the chemoenzymatic synthesis of a spacer-containing tetrasaccharide fragment of the CPS of $S$. pneumoniae type 14, corresponding with one repeating unit $\{\beta-\mathrm{D}-\mathrm{Gal} p-(1 \rightarrow$ 4)- $\beta$-D-Glc $p-(1 \rightarrow 6)-[\beta-\mathrm{D}-\mathrm{Gal} p-(1 \rightarrow 4)]-\beta-\mathrm{D}-\mathrm{Glc} p \mathrm{NAc}\}$, as well as mimics of the CPS. ${ }^{11,12}$ The tetrasaccharidecontaining neoglycoconjugate $\left(\mathrm{CRM}_{197}\right.$ as carrier protein) showed particularly promising immunological data when tested in mice models. ${ }^{13}$ Based on these results, it was decided to synthesize a series of longer oligosaccharide fragments of the CPS for more detailed immunological studies. Recently, we have described the chemo-enzymatic synthesis of four oligosaccharides with a 6-aminohexyl spacer, varying in length between one and two repeating units. ${ }^{14,15}$ Here, we report the chemo-enzymatic synthesis of another four oligosac- 
charides, containing the same spacer. The whole series of eight spacered oligosaccharides will form an excellent panel to investigate the structural parameters influencing immunogenicity.

\section{Results and discussion}

For the organic synthesis of the structurally closely related compounds 5-8, a series of broadly applicable building blocks, 9, 13, 24, and 37, were designed. Condensation of these key building blocks with appropriate acceptor or donor building blocks gave, after deprotection, the aimed linear oligosaccharides (5-8), which were used as acceptor substrates for $\beta-1,4-$ galactosyltransferase (EC 2.4.1.22) from bovine milk to give the desired title compounds 1-4 (Scheme 1).

\subsection{Synthesis of tetrasaccharide fragment 1}

The linear trisaccharide backbone $\mathbf{5}$ was prepared via two different routes (Scheme 2). For the first route, disaccharide donor 13 was needed. Coupling of 2,3,4,6tetra- $O$-acetyl- $\alpha$-D-glucopyranosyl trichloroacetimidate (9) ${ }^{16}$ to allyl 2-deoxy-3,4-di- $O$ - $p$-methylbenzoyl-2- phthalimido- $\beta$-D-glucopyranoside $(\mathbf{1 0})^{11}$ in dichloromethane at $0{ }^{\circ} \mathrm{C}$, using 1 equivalent silver trifluoromethanesulfonate (AgOTf) as a catalyst, gave disaccharide 11 in $62 \%$ yield. A small amount of a side product, the acetylated acceptor, was also isolated. Side product formation was much higher when using $10 \%$ trimethylsilyl trifluoromethanesulfonate (TMSOTf) as a catalyst $\left(0{ }^{\circ} \mathrm{C}: 34 \% 11,53 \%\right.$ side product; $-40{ }^{\circ} \mathrm{C}: 50 \%$ 11, 25\% side product). De-allylation of $\mathbf{1 1}$ by using palladium(II)chloride, sodium acetate, and acetic acid in an ultrasonic bath $(\rightarrow \mathbf{1 2}, 70 \%)$, followed by imidation of the anomeric center, using trichloroacetonitrile with 1,8-diazabicyclo[5.4.0]undec-7-ene (DBU) as a catalyst, gave disaccharide donor $13(76 \%)$. Coupling of 13 to 6azidohexyl 4- $O$-acetyl-2,6-di- $O$-benzyl- $\beta$-D-galactopyranoside (14) ${ }^{14}$ at $-70{ }^{\circ} \mathrm{C}$ in dichloromethane, using $10 \%$ trimethylsilyl trifluoromethanesulfonate as a catalyst, gave trisaccharide 21 (73\%).

In the first step of an alternative route to trisaccharide 21, 3,4,6-tri- $O$-acetyl-2-deoxy-2-phthalimido- $\beta$-D-glucopyranosyl trichloroacetimidate $(\mathbf{1 5})^{17}$ was coupled to galactose acceptor 14 , at $-70{ }^{\circ} \mathrm{C}$ in dichloromethane, using $10 \%$ trimethylsilyl trifluoromethanesulfonate as a catalyst, to give disaccharide $\mathbf{1 6}$ in $86 \%$ yield. Mild de$\mathrm{O}$-acetylation of $\mathbf{1 6}$, using sodium methoxide at $\mathrm{pH} 8$,
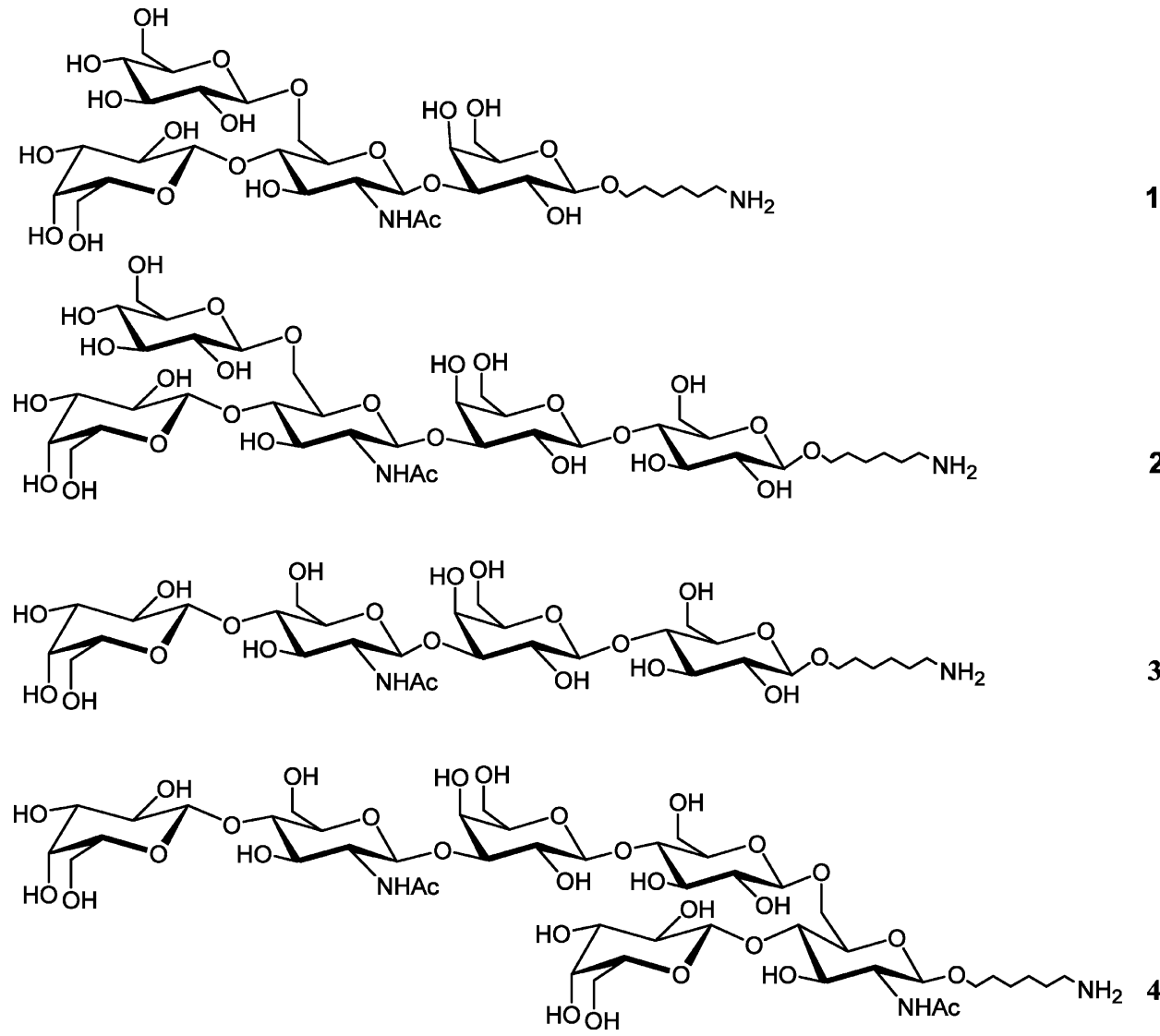

Scheme 1. Overview of 6-aminohexyl-spacered oligosaccharides 1-4, representing fragments of the repeating unit of the $S$. pneumoniae serotype 14 capsular polysaccharide. 

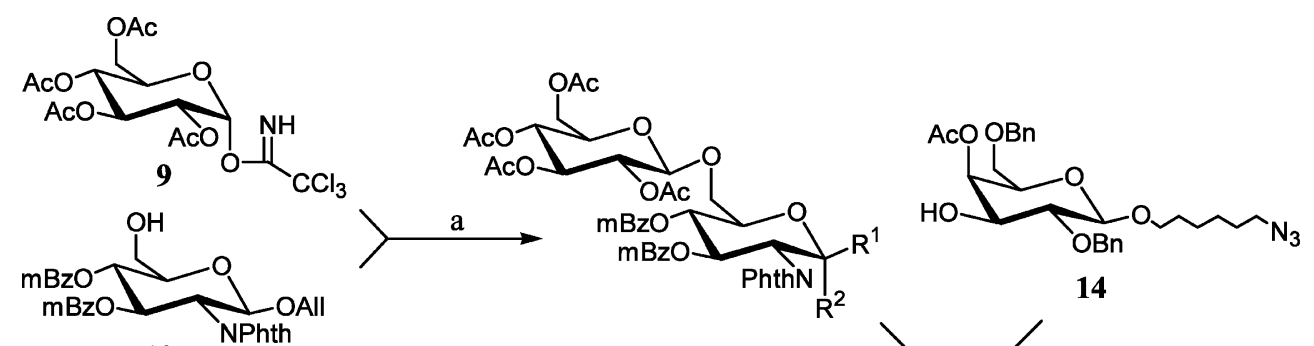

10

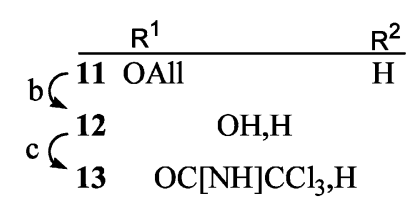

5 e
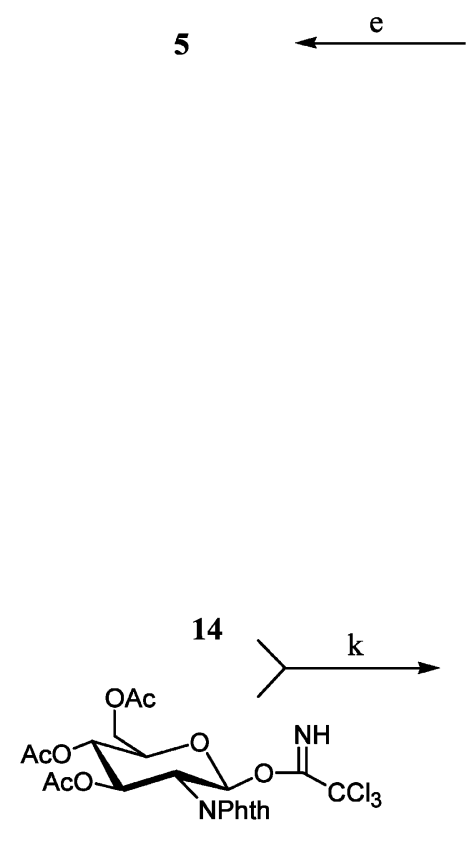

15

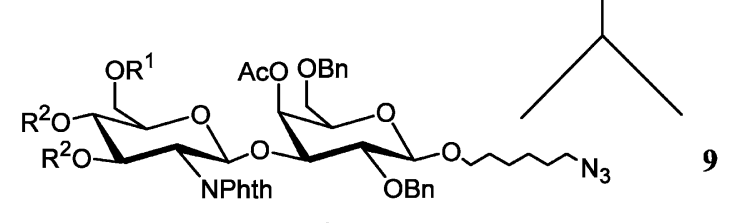

\begin{tabular}{lll} 
& $\mathrm{R}^{1}$ & $\mathrm{R}^{2}$ \\
\hline $\mathbf{1 6}$ & $\mathrm{Ac}$ & $\mathrm{Ac}$ \\
$\mathbf{1 7}$ & $\mathrm{H}$ & $\mathrm{H}$ \\
$\mathbf{1 8}$ & $\mathrm{tBdPSi}$ & $\mathrm{H}$ \\
$\mathbf{1 9}$ & $\mathrm{tBdPSi}$ & $\mathrm{mBz}$ \\
$\mathbf{2 0}$ & $\mathrm{H}$ & $\mathrm{mBz}$
\end{tabular}

Scheme 2. Synthesis of the linear trisaccharide backbone 5: (a) 1 equiv $\mathrm{AgOTf}, \mathrm{CH}_{2} \mathrm{Cl}_{2}, 0{ }^{\circ} \mathrm{C}, 62 \%$; (b) $\mathrm{Pd}(\mathrm{II}) \mathrm{Cl}{ }_{2}, \mathrm{NaOAc}, \mathrm{AcOH}$, $70 \%$; (c) $\mathrm{Cl}_{3} \mathrm{CCN}, \mathrm{DBU}, \mathrm{CH}_{2} \mathrm{Cl}_{2}, 76 \%$; (d) $10 \%$ TMSOTf, $\mathrm{CH}_{2} \mathrm{Cl}_{2},-70{ }^{\circ} \mathrm{C}, 73 \%$; (e) $10 \% \mathrm{Pd}-\mathrm{C}, \mathrm{H}_{2}$, water, tert $-\mathrm{BuOH}$, aq $25 \%$ $\mathrm{NH}_{3} / 10 \% \mathrm{Pd}-\mathrm{C}$, AcOH, 69\%; (f) NaOMe (pH 10), $\mathrm{MeOH}, \mathrm{CH}_{2} \mathrm{Cl}_{2}$; (g) $\mathrm{NH}_{2} \mathrm{CH}_{2} \mathrm{CH}_{2} \mathrm{NH}_{2}, 1-\mathrm{BuOH}, 80{ }^{\circ} \mathrm{C}$; (h) pyridine, Ac ${ }_{2} \mathrm{O}$, $80 \%$ over three steps; (i) $\mathrm{NaOMe}\left(\mathrm{pH}\right.$ 9), $\mathrm{MeOH}, \mathrm{CH}_{2} \mathrm{Cl}_{2}, 97 \%$; (j) 10\% TMSOTf, $\mathrm{CH}_{2} \mathrm{Cl}_{2},-40{ }^{\circ} \mathrm{C}, 56 \%$; (k) $10 \% \mathrm{TMSOTf}$, $\mathrm{CH}_{2} \mathrm{Cl}_{2},-70{ }^{\circ} \mathrm{C}, 86 \%$; (1) $\mathrm{NaOMe}\left(\mathrm{pH} 8\right.$ ), $\mathrm{MeOH}, \mathrm{CH}_{2} \mathrm{Cl}_{2}, 91 \%$; (m) $t$-BdPSiCl, DMAP, Et 3 N, $\mathrm{CH}_{2} \mathrm{Cl}_{2}, \mathrm{pyridine}, 83 \%$; (n) $p$ $\mathrm{mBzCl}$, pyridine, $\mathrm{CH}_{2} \mathrm{Cl}_{2}, 90 \%$; (o) $\mathrm{AcCl}, \mathrm{MeOH}$, toluene, $0{ }^{\circ} \mathrm{C}, 81 \%$.

gave $17(91 \%)$ with a retention of the acetyl group at galactose O-4. Selective introduction of a tert-butyldiphenylsilyl group ( $t \mathrm{BdPSi})$ at the primary hydroxyl function of 17 using tert-butyldiphenylsilyl chloride and a catalytic amount of 4-dimethylaminopyridine (DMAP) $(\rightarrow \mathbf{1 8}, 83 \%)$, followed by $p$-methylbenzoyla- tion $(\mathrm{mBz})$ of the two remaining hydroxyl groups, using $p$-methylbenzoyl chloride in pyridine, gave $19(90 \%)$. Finally, removal of the tert-butyldiphenylsilyl group under mild acidic conditions afforded disaccharide acceptor $\mathbf{2 0}$ in $81 \%$ yield. A minor side product, the O-de-acetylated analogue of $\mathbf{2 0}$, was isolated also (14\% 
yield). Coupling of glucose donor $\mathbf{9}$ to disaccharide $\mathbf{2 0}$ in dichloromethane at $-40{ }^{\circ} \mathrm{C}$, using $10 \%$ trimethylsilyl trifluoromethanesulfonate as a catalyst, gave trisaccharide 21 in 56\% yield. O-De-acylation of 21 using sodium methoxide at $\mathrm{pH} 10$, followed by N-de-phthaloylation using 1,2-diaminoethane in 1-butanol at $80^{\circ} \mathrm{C}$, and subsequent $\mathrm{N}, \mathrm{O}$-acetylation using acetic anhydride in pyridine yielded $\mathbf{2 2}$ in $80 \%$ yield over three steps. The Oacetylation step was carried out to facilitate chromatographic purification. O-De-acetylation of 22 with sodium methoxide at $\mathrm{pH} 9$ gave crude $\mathbf{2 3}$, and after reduction of the azido function using $10 \% \mathrm{Pd}-\mathrm{C}$ and $\mathrm{H}_{2}$ in the presence of ammonia, and subsequent debenzylation using $10 \% \mathrm{Pd}-\mathrm{C}$ and $\mathrm{H}_{2}$ in the presence of acetic acid, linear trisaccharide backbone $\mathbf{5}$ was obtained in a yield of $69 \%$. Tetrasaccharide 1 was synthesized in $54 \%$ yield by the transfer of galactose from UDP-galactose to O-4 of the $N$-acetyl- $\beta$-D-glucosamine residue of $\mathbf{5}$ by using bovine milk $\beta-1,4$-galactosyltransferase as a catalyst (Scheme 3). As a result of the enzymatic galactosylation of the terminal glucose residue, the previously described pentasaccharide 6-aminohexyl $\beta$-D-galactopyranosyl-( $(1 \rightarrow 4)-\beta$-D-glucopyranosyl- $(1 \rightarrow 6)$ - $[\beta$-D-galactopyranosyl- $(1 \rightarrow 4)]$-2-acetamido-2-deoxy- $\beta$-D-glucopyranosyl- $(1 \rightarrow 3)-\beta$-D-galactopyranoside $(\mathbf{4 7})^{14}$ was obtained as a side product (35\%) (Scheme 3 ). So far, the enzymatic galactosylation of glucose by $\beta-1,4-$ galactosyltransferase has only been shown to proceed in the presence of $\alpha$-lactalbumin (lactose synthase complex). ${ }^{18}{ }^{1} \mathrm{H}$ NMR data of $\mathbf{5}$ and $\mathbf{1}$, derived from 2D TOCSY and ROESY measurements, are presented in Tables 1 and 2, respectively.

Table 1

$500 \mathrm{MHz}{ }^{1} \mathrm{H}$ NMR data (TOCSY, ROESY) of 5 at $300 \mathrm{~K}$ (in ppm)

\begin{tabular}{|c|c|c|c|}
\hline \multirow[t]{2}{*}{ Proton } & \multicolumn{3}{|l|}{$\delta_{\mathrm{H}}$} \\
\hline & $\begin{array}{l}\text { Gal } \\
\text { I }\end{array}$ & GlcNAc II & $\begin{array}{l}\text { Glc } \\
\text { III }\end{array}$ \\
\hline $\mathrm{H}-1$ & 4.37 & 4.70 & 4.50 \\
\hline $\mathrm{H}-2$ & 3.57 & 3.76 & 3.31 \\
\hline $\mathrm{H}-3$ & 3.72 & 3.56 & 3.50 \\
\hline $\mathrm{H}-4$ & 4.15 & n.d. ${ }^{a}$ & 3.39 \\
\hline H-5 & n.d. & 3.61 & 3.48 \\
\hline H-6a & n.d. & 4.22 & 3.92 \\
\hline H-6b & n.d. & 3.89 & 3.72 \\
\hline $\mathrm{O}\left(\mathrm{CH}_{2}\right)_{2}\left(\mathrm{CH}_{2}\right)_{2}\left(\mathrm{CH}_{2}\right)_{2} \mathrm{ND}_{2}$ & & $\begin{array}{l}1.39-1.41(4 \\
\mathrm{H})\end{array}$ & \\
\hline $\mathrm{OCH}_{2} \mathrm{CH}_{2}\left(\mathrm{CH}_{2}\right)_{2} \mathrm{CH}_{2} \mathrm{CH}_{2} \mathrm{ND}_{2}$ & & $\begin{array}{l}1.64-1.65(4 \\
\mathrm{H})\end{array}$ & \\
\hline $\mathrm{CH}_{2} \mathrm{ND}_{2}$ & & 2.96 & \\
\hline $\mathrm{OCH}_{2}\left(\mathrm{CH}_{2}\right)_{5} \mathrm{ND}_{2}$ & & $3.68,3.92$ & \\
\hline $\mathrm{NDCOCH}_{3}$ & & 2.04 & \\
\hline
\end{tabular}

${ }^{a}$ n.d., not determined.

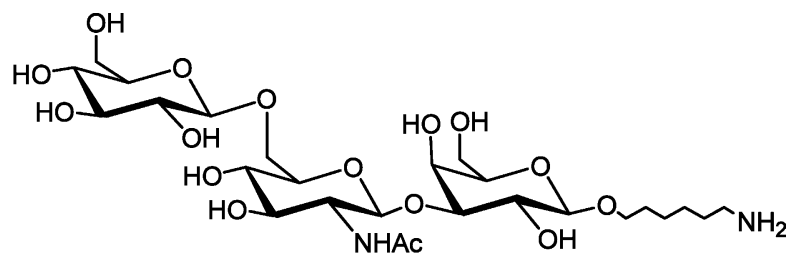

5

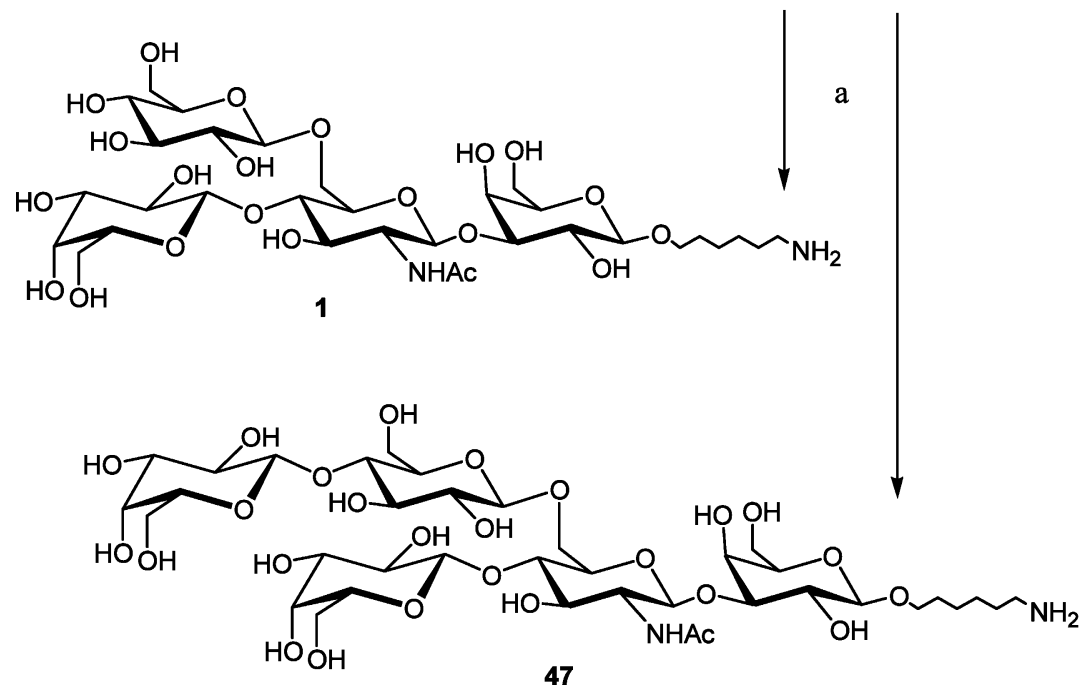

Scheme 3. Synthesis of tetrasaccharide 1 and pentasaccharide 47: (a) 1.4 equiv UDP-Gal, aq $50 \mathrm{mM}$ sodium cacodylate buffer (pH 7.5), $3 \mathrm{U} \beta$-1,4-galactosyltransferase, $14 \mathrm{U}$ alkaline phosphatase, $37^{\circ} \mathrm{C}, 54 \%$ (1); $35 \%$ (47). 
Table 2

$500 \mathrm{MHz}{ }^{1} \mathrm{H}$ NMR data (TOCSY, ROESY) of 1 at $300 \mathrm{~K}$ (in ppm)

\begin{tabular}{|c|c|c|c|c|}
\hline \multirow[t]{2}{*}{ Proton } & \multicolumn{4}{|l|}{$\delta_{\mathrm{H}}$} \\
\hline & Gal I ${ }^{\mathrm{a}}$ & GlcNAc II & Glc III & Gal IV $^{\mathrm{b}}$ \\
\hline $\mathrm{H}-1$ & 4.38 & 4.72 & 4.52 & 4.54 \\
\hline $\mathrm{H}-2$ & 3.55 & 3.81 & 3.32 & 3.54 \\
\hline $\mathrm{H}-3$ & 3.70 & 3.73 & 3.50 & 3.67 \\
\hline $\mathrm{H}-4$ & 4.16 & 3.87 & 3.39 & 3.92 \\
\hline $\mathrm{H}-5$ & n.d. ${ }^{\mathrm{c}}$ & 3.73 & 3.47 & n.d. \\
\hline H-6a & n.d. & 4.28 & 3.86 & n.d. \\
\hline $\mathrm{H}-6 \mathrm{~b}$ & n.d. & 3.95 & 3.72 & n.d. \\
\hline $\mathrm{O}\left(\mathrm{CH}_{2}\right)_{2}\left(\mathrm{CH}_{2}\right)_{2}\left(\mathrm{CH}_{2}\right)_{2} \mathrm{ND}_{2}$ & \multicolumn{4}{|c|}{$1.39-1.41(4 \mathrm{H})$} \\
\hline $\mathrm{OCH}_{2} \mathrm{CH}_{2}\left(\mathrm{CH}_{2}\right)_{2} \mathrm{CH}_{2} \mathrm{CH}_{2} \mathrm{ND}_{2}$ & \multicolumn{4}{|c|}{$1.63-1.64(4 \mathrm{H})$} \\
\hline $\mathrm{CH}_{2} \mathrm{ND}_{2}$ & \multicolumn{4}{|c|}{2.96} \\
\hline $\mathrm{OCH}_{2}\left(\mathrm{CH}_{2}\right)_{5} \mathrm{ND}_{2}$ & \multicolumn{4}{|c|}{$3.67,3.92$} \\
\hline $\mathrm{NDCOCH}_{3}$ & \multicolumn{4}{|c|}{2.03} \\
\hline
\end{tabular}

${ }^{\text {a }} \mathrm{Gal}\left(\beta 1-\mathrm{O}\left(\mathrm{CH}_{2}\right)_{6} \mathrm{NH}_{2}\right)$.

b $\mathrm{Gal}(\beta 1-4) \mathrm{GlcNAc}$.

c n.d., not determined.

\subsection{Synthesis of pentasaccharide fragment 2}

The linear tetrasaccharide backbone $\mathbf{6}$ was prepared via two different routes (Scheme 4). In the first route, condensation of disaccharide donor 13 with 6-azidohexyl (4- $O$-acetyl-2,6-di- $O$-benzyl- $\beta$-D-galactopyranosyl)-( $1 \rightarrow 4)$-2,3,6-tri- $O$-benzyl- $\beta$-D-glucopyranoside $(\mathbf{2 4})^{14}$ in dichloromethane at $-70^{\circ} \mathrm{C}$, using $10 \%$ trimethylsilyl trifluoromethanesulfonate as a catalyst, afforded tetrasaccharide $\mathbf{3 0}$ in $83 \%$ yield. An alternative route to tetrasaccharide $\mathbf{3 0}$ involved the condensation of glucose donor 9 (see Scheme 2) with trisaccharide acceptor 6-azidohexyl (2-deoxy-3,4-di- $O$ - $p$-methylbenzoyl-2-phthalimido- $\beta$-D-glucopyranosyl)-( $1 \rightarrow 3)-(4-O$ acetyl-2,6-di- $O$-benzyl- $\beta$-D-galactopyranosyl)-( $1 \rightarrow 4)$ $2,3,6$-tri- $O$-benzyl- $\beta$-D-glucopyranoside (29). As a first step in the synthesis of $\mathbf{2 9}$, glucosamine donor $\mathbf{1 5}^{17}$ (see Scheme 2) was coupled to lactose acceptor $\mathbf{2 4}$ in dichloromethane at $-70{ }^{\circ} \mathrm{C}$, using $10 \%$ trimethylsilyl trifluoromethanesulfonate as a catalyst, to give trisaccharide 25 in $97 \%$ yield. Mild O-de-acetylation of 25, using sodium methoxide at $\mathrm{pH} 8$, gave 26 (78\%), with a retention of the acetyl group at galactose O-4. Selective introduction of a tert-butyldiphenylsilyl group at the primary hydroxyl function of $\mathbf{2 6}$ using tert-butyldiphenylsilyl chloride and a catalytic amount of 4-dimethylaminopyridine $(\rightarrow \mathbf{2 7}, 84 \%)$, followed by $p$ methylbenzoylation of the two remaining hydroxyl groups, using $p$-methylbenzoyl chloride in pyridine gave $28(91 \%)$. Finally, selective removal of the silyl group, using a 1:1 mixture of 1.0 M TBAF in THF and $\mathrm{AcOH}$ at $\mathrm{pH}$ 6, gave 29 in $89 \%$ yield. Coupling of donor 9 to acceptor 29 in dichloromethane at $-40{ }^{\circ} \mathrm{C}$, using $10 \%$ trimethylsilyl trifluoromethanesulfonate as a cata- lyst gave tetrasaccharide $\mathbf{3 0}$ (58\%). O-De acylation of $\mathbf{3 0}$ using sodium methoxide at $\mathrm{pH} 10$, followed by $\mathrm{N}$-dephthaloylation using 1,2-diaminoethane in 1-butanol at $80{ }^{\circ} \mathrm{C}$, and subsequent $\mathrm{N}, \mathrm{O}$-acetylation using acetic anhydride in pyridine yielded 31 in $88 \%$ yield over three steps. O-De-acetylation of $\mathbf{3 1}$ with sodium methoxide at $\mathrm{pH} 10$ gave crude 32. After reduction of the azido function using $10 \% \mathrm{Pd}-\mathrm{C}$ and $\mathrm{H}_{2}$ in the presence of ammonia, and subsequent debenzylation using $10 \% \mathrm{Pd}-$ $\mathrm{C}$ and $\mathrm{H}_{2}$ in the presence of acetic acid, the linear tetrasaccharide backbone 6 was obtained in a yield of $81 \%$. Pentasaccharide 2 was synthesized in $65 \%$ yield by the transfer of galactose from UDP-galactose to O-4 of the $N$-acetyl- $\beta$-D-glucosamine residue of $\mathbf{6}$ by using bovine milk $\beta$-1,4-galactosyltransferase as a catalyst (Scheme 5). As a digalactosylated side product the previously described hexasaccharide 6-aminohexyl $\beta$-Dgalactopyranosyl-( $1 \rightarrow 4)$ - $\beta$-D-glucopyranosyl- $(1 \rightarrow 6)$-[ $[\beta$ D-galactopyranosyl- $(1 \rightarrow 4)]$-2-acetamido-2-deoxy- $\beta$-Dglucopyranosyl-( $\rightarrow 3)$ - $\beta$-D-galactopyranosyl- $(1 \rightarrow 4)-\beta$ D-glucopyranoside (48) ${ }^{14}(6 \%$; Scheme 5$)$ was obtained. ${ }^{1}$ H NMR data of $\mathbf{6}$ and 2, derived from 2D TOCSY and ROESY measurements, are presented in Tables 3 and 4, respectively.

\subsection{Synthesis of tetrasaccharide fragment 3}

The linear trisaccharide backbone 7 was prepared via two different routes (Scheme 6). For the first route, trisaccharide donor 37 was needed. Debenzylation of (3,4,6-tri- $O$-acetyl-2-deoxy-2-phthalimido- $\beta$-D-glucopyranosyl)-( $1 \rightarrow 3)$-(4- $O$-acetyl-2,6-di- $O$-benzyl- $\beta$-D-galactopyranosyl)-( $1 \rightarrow 4)$-1,2,3,6-tetra- $O$-benzyl- $\beta$-D-glucopyranoside (34), ${ }^{15}$ using $10 \% \mathrm{Pd}-\mathrm{C}$ and $\mathrm{H}_{2}$ in the 
13
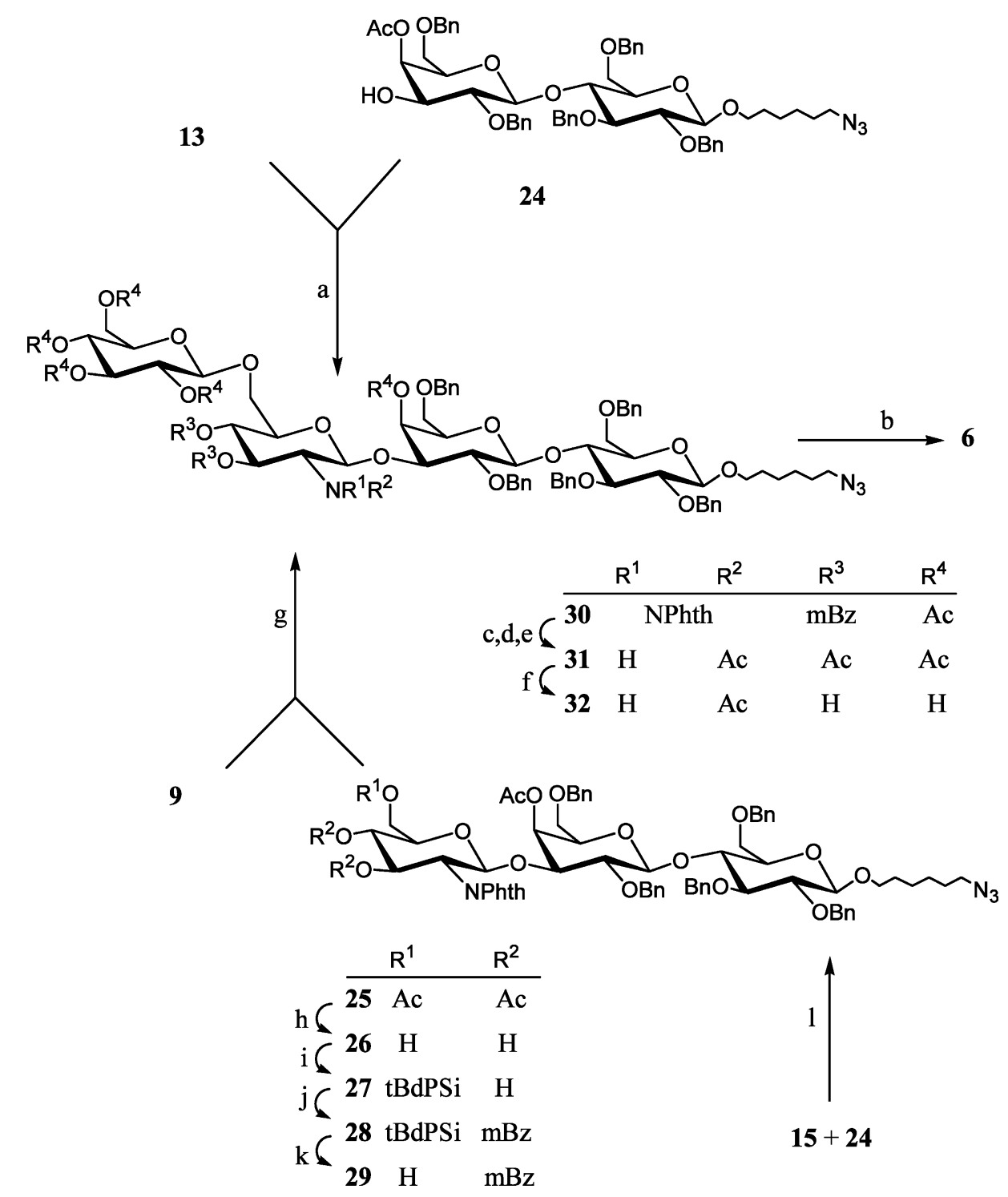

Scheme 4. Synthesis of the linear tetrasaccharide backbone 6: (a) $10 \%$ TMSOTf, $\mathrm{CH}_{2} \mathrm{Cl}_{2},-70{ }^{\circ} \mathrm{C}, 83 \%$; (b) $10 \% \mathrm{Pd}-\mathrm{C}, \mathrm{H}_{2}$, water, tert-BuOH, aq $25 \% \mathrm{NH}_{3} / 10 \% \mathrm{Pd}-\mathrm{C}, \mathrm{AcOH}, 81 \%$; (c) $\mathrm{NaOMe}$ (pH 10), $\mathrm{MeOH}, \mathrm{CH}_{2} \mathrm{Cl}_{2}$; (d) $\mathrm{NH}_{2} \mathrm{CH}_{2} \mathrm{CH}_{2} \mathrm{NH}_{2}, 1-\mathrm{BuOH}, 80^{\circ} \mathrm{C}$; (e) pyridine, $\mathrm{Ac}_{2} \mathrm{O}, 88 \%$ over three steps; (f) $\mathrm{NaOMe}\left(\mathrm{pH} 10\right.$ ), $\mathrm{MeOH}, \mathrm{CH}_{2} \mathrm{Cl}_{2}$, quantitative; (g) $10 \%$ TMSOTf, $\mathrm{CH}_{2} \mathrm{Cl}_{2},-40{ }^{\circ} \mathrm{C}, 58 \%$; (h) NaOMe (pH 8), $\mathrm{MeOH}, \mathrm{CH}_{2} \mathrm{Cl}_{2}, 78 \%$; (i) $t$ - $\mathrm{BdPSiCl}$, DMAP, $\mathrm{Et}_{3} \mathrm{~N}, \mathrm{CH}_{2} \mathrm{Cl}_{2}$, pyridine, $84 \%$; (j) $p$-mBzCl, pyridine, $\mathrm{CH}_{2} \mathrm{Cl}_{2}$, $91 \%$; (k) $1.0 \mathrm{M}$ TBAF in THF, AcOH (pH 6), 89\%; (l) 10\% TMSOTf, $\mathrm{CH}_{2} \mathrm{Cl}_{2},-70{ }^{\circ} \mathrm{C}, 97 \%$.

presence of acetic acid, followed by O-acetylation using acetic anhydride in pyridine afforded 35 in $66 \%$ yield over two steps. Selective O-de-acetylation of the anomeric center of 35, using hydrazinium acetate in $N, N$ dimethylformamide $(\rightarrow \mathbf{3 6}, 89 \%)$, and subsequent imidation using trichloroacetonitrile with 1,8-diazabicyclo[5.4.0]undec-7-ene as a catalyst gave trisaccharide donor 37 in $69 \%$ yield. Condensation of $\mathbf{3 7}$ with 6-azido1-hexanol (33) in dichloromethane at $0{ }^{\circ} \mathrm{C}$, using 1 equivalent silver trifluoromethanesulfonate as a catalyst, gave trisaccharide $\mathbf{3 8}$ in $22 \%$ yield only. The low yield was due to orthoester formation, and coupling attempts at different temperatures and/or applying trimethylsilyl trifluoromethanesulfonate as a catalyst did not improve the yield. O-De-acetylation of $\mathbf{3 8}$, using sodium methoxide ( $\mathrm{pH} 10$ ), followed by N-de-phthaloylation using 1,2-diaminoethane in 1-butanol at $80^{\circ} \mathrm{C}$, and subsequent $\mathrm{N}, \mathrm{O}$-acetylation using acetic anhydride in pyridine yielded 39 ( $77 \%$ over three steps). De-O-acetylation of $\mathbf{3 9}$ with sodium methoxide at $\mathrm{pH} 10(\rightarrow \mathbf{4 0}, 55 \%)$, and reduction of the azido function using $10 \% \mathrm{Pd}-\mathrm{C}$ and $\mathrm{H}_{2}$ in the presence of ammonia, gave the linear trisaccharide backbone 7 (83\%). ${ }^{1} \mathrm{H}$ NMR data of $\mathbf{4 0}$ and $\mathbf{7}$, derived from 2D TOCSY and ROESY measurements, are presented in Tables 5 and 6 , respectively. In an alternative route to linear backbone 7 , trisaccharide 25 was O-de-acetylated using sodium methoxide ( $\mathrm{pH} 10)$, followed by N-de-phthaloylation using 1,2-diami- 

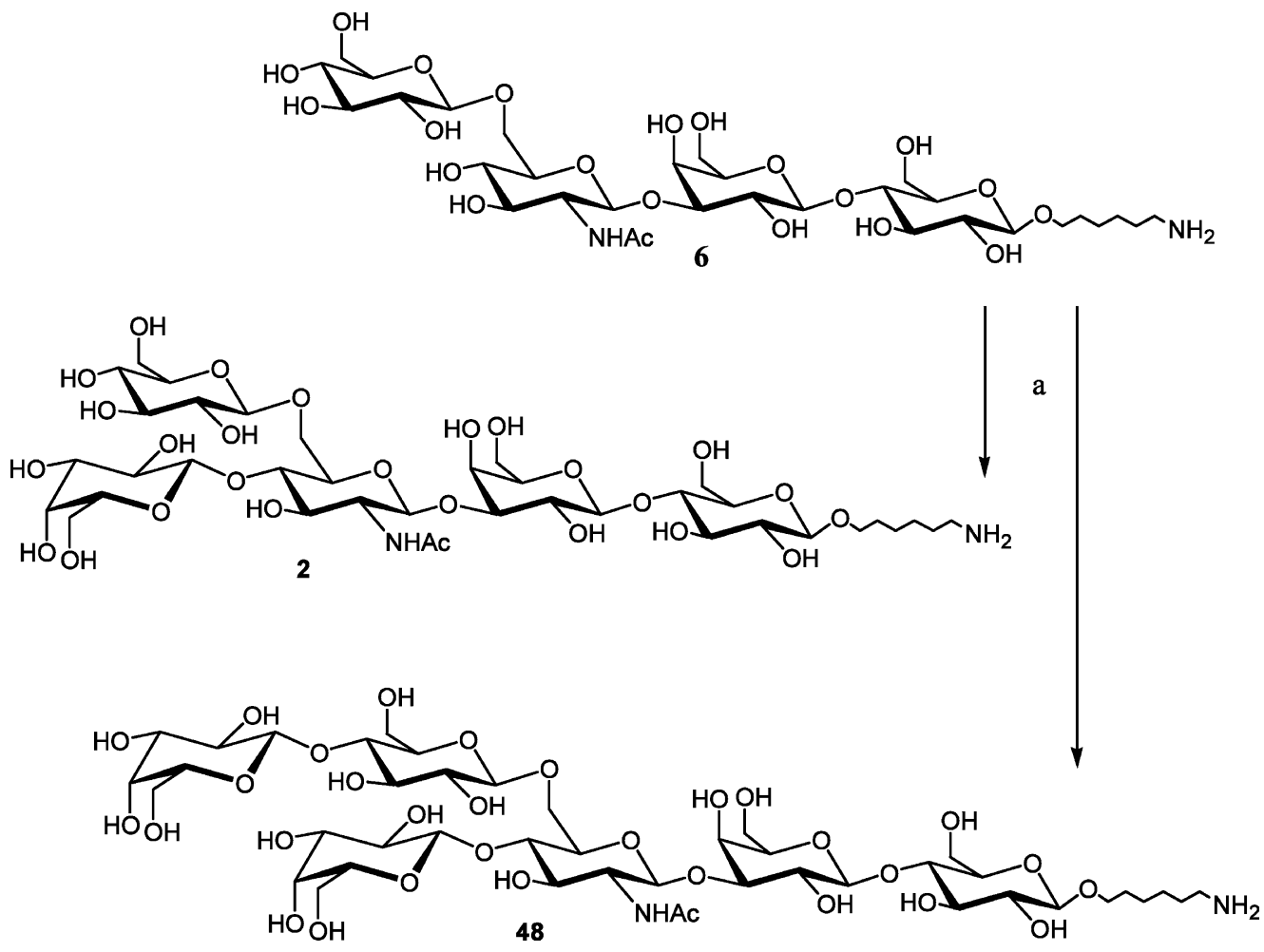

Scheme 5. Synthesis of pentasaccharide 2 and hexasaccharide 48: (a) 1.4 equiv UDP-Gal, aq 50 mM sodium cacodylate buffer (pH 7.5), $3 \mathrm{U} \beta$-1,4-galactosyltransferase, $14 \mathrm{U}$ alkaline phosphatase, $37^{\circ} \mathrm{C}, 65 \%(2) ; 6 \%$ (48).

Table 3

$500 \mathrm{MHz}{ }^{1} \mathrm{H}$ NMR data (TOCSY, ROESY) of 6 at $300 \mathrm{~K}$ (in ppm)

\begin{tabular}{|c|c|c|c|c|}
\hline \multirow[t]{2}{*}{ Proton } & \multicolumn{4}{|l|}{$\delta_{\mathrm{H}}$} \\
\hline & $\begin{array}{l}\text { Glc } \\
\mathrm{I}^{\mathrm{a}}\end{array}$ & $\begin{array}{l}\text { Gal } \\
\text { II }\end{array}$ & $\begin{array}{l}\text { GlcNAc } \\
\text { III }\end{array}$ & $\begin{array}{l}\text { Glc } \\
\mathrm{IV}^{\mathrm{b}}\end{array}$ \\
\hline $\mathrm{H}-1$ & 4.47 & 4.43 & 4.69 & 4.49 \\
\hline $\mathrm{H}-2$ & 3.29 & 3.58 & 3.76 & 3.30 \\
\hline $\mathrm{H}-3$ & 3.63 & 3.72 & 3.57 & 3.49 \\
\hline $\mathrm{H}-4$ & 3.63 & 4.15 & n.d. ${ }^{c}$ & 3.38 \\
\hline $\mathrm{H}-5$ & 3.60 & n.d. & 3.61 & 3.47 \\
\hline $\mathrm{H}-6 \mathrm{a}$ & 3.97 & n.d. & 4.20 & 3.92 \\
\hline $\mathrm{H}-6 \mathrm{~b}$ & & n.d. & 3.88 & 3.72 \\
\hline $\mathrm{O}\left(\mathrm{CH}_{2}\right)_{2}\left(\mathrm{CH}_{2}\right)_{2}\left(\mathrm{CH}_{2}\right)_{2} \mathrm{ND}_{2}$ & & & $\begin{array}{l}1.39-1.41 \\
(4 \mathrm{H})\end{array}$ & \\
\hline $\mathrm{OCH}_{2} \mathrm{CH}_{2}\left(\mathrm{CH}_{2}\right)_{2} \mathrm{CH}_{2} \mathrm{CH}_{2} \mathrm{ND}_{2}$ & & & $\begin{array}{l}1.64-1.65 \\
(4 \mathrm{H})\end{array}$ & \\
\hline $\mathrm{CH}_{2} \mathrm{ND}_{2}$ & & & 2.95 & \\
\hline $\mathrm{OCH}_{2}\left(\mathrm{CH}_{2}\right)_{5} \mathrm{ND}_{2}$ & & & $3.68,3.92$ & \\
\hline $\mathrm{NDCOCH}_{3}$ & & & 2.03 & \\
\hline
\end{tabular}

a $\mathrm{Glc}\left(\beta 1-\mathrm{O}\left(\mathrm{CH}_{2}\right)_{6} \mathrm{NH}_{2}\right)$.

b $\operatorname{Glc}(\beta 1-6)$ GlcNAc.

c n.d., not determined.

noethane in 1-butanol at $80{ }^{\circ} \mathrm{C}$, and subsequent $\mathrm{N}, \mathrm{O}$ acetylation using acetic anhydride in pyridine afforded
41 (78\% over three steps). De-O-acetylation of $\mathbf{4 1}$ with sodium methoxide ( $\mathrm{pH} 10)$ gave crude $\mathbf{4 2}$, and after reduction of the azido function of $\mathbf{4 2}$ using $10 \% \mathrm{Pd}-\mathrm{C}$ and $\mathrm{H}_{2}$ in the presence of ammonia, and subsequent debenzylation using $10 \% \mathrm{Pd}-\mathrm{C}$ and $\mathrm{H}_{2}$ in the presence of acetic acid, linear trisaccharide backbone 7 was obtained in a yield of $68 \%$. Tetrasaccharide 3 was synthesized in $94 \%$ yield by the transfer of galactose from UDP-galactose to O- 4 of the $N$-acetyl- $\beta$-D-glucosamine residue of 7 by using bovine milk $\beta-1,4-$ galactosyltransferase as a catalyst (Scheme 7). 2D TOCSY and ROESY measurements confirmed the structure of 3 (Table 7). Chemical- and chemo-enzymatic syntheses of 7 containing other functionalities at the anomeric center have been described earlier. ${ }^{19}$

\subsection{Synthesis of hexasaccharide fragment 4}

To obtain the linear tetrasaccharide backbone $\mathbf{8}$, as a first step trisaccharide donor 37 (see Scheme 6) was coupled with 6-azidohexyl 2-deoxy-3,4-di- $O$ - $p$-methylbenzoyl-2-phthalimido- $\beta$-D-glucopyranoside

$(43)^{15}$

(Scheme 8) in dichloromethane at $0{ }^{\circ} \mathrm{C}$, using $15 \%$ trimethylsilyl trifluoromethanesulfonate as a catalyst, to give tetrasaccharide $\mathbf{4 4}$ in $34 \%$ yield. O-De-acylation of $\mathbf{4 4}$ using sodium methoxide ( $\mathrm{pH} 10$ ), followed by $\mathrm{N}$ de-phthaloylation using 1,2-diaminoethane in 1-butanol at $90{ }^{\circ} \mathrm{C}$, and subsequent $\mathrm{N}, \mathrm{O}$-acetylation using acetic 
Table 4

$500 \mathrm{MHz}{ }^{1} \mathrm{H}$ NMR data (TOCSY, ROESY) of 2 at $300 \mathrm{~K}$ (in ppm)

\begin{tabular}{|c|c|c|c|c|c|}
\hline \multirow[t]{2}{*}{ Proton } & \multicolumn{5}{|l|}{$\delta_{\mathrm{H}}$} \\
\hline & Glc I ${ }^{a}$ & Gal II $^{\mathrm{b}}$ & GlcNAc III & Glc IV $^{\mathrm{c}}$ & Gal V ${ }^{d}$ \\
\hline $\mathrm{H}-1$ & 4.48 & 4.43 & 4.71 & 4.53 & 4.52 \\
\hline $\mathrm{H}-2$ & 3.29 & 3.59 & 3.81 & 3.33 & 3.54 \\
\hline H-3 & 3.64 & 3.72 & 3.73 & 3.50 & 3.67 \\
\hline $\mathrm{H}-4$ & n.d. ${ }^{\mathrm{e}}$ & 4.16 & 3.87 & 3.40 & 3.93 \\
\hline H-5 & n.d. & n.d. & 3.73 & 3.46 & n.d. \\
\hline H-6a & 3.95 & n.d. & 4.27 & 3.86 & n.d. \\
\hline $\mathrm{H}-6 \mathrm{~b}$ & 3.78 & n.d. & 3.95 & 3.73 & n.d. \\
\hline $\mathrm{O}\left(\mathrm{CH}_{2}\right)_{2}\left(\mathrm{CH}_{2}\right)_{2}\left(\mathrm{CH}_{2}\right)_{2} \mathrm{ND}_{2}$ & & & $1.39-1.41(4 \mathrm{H})$ & & \\
\hline $\mathrm{OCH}_{2} \mathrm{CH}_{2}\left(\mathrm{CH}_{2}\right)_{2} \mathrm{CH}_{2} \mathrm{CH}_{2} \mathrm{ND}_{2}$ & & & $1.64-1.65(4 \mathrm{H})$ & & \\
\hline $\mathrm{CH}_{2} \mathrm{ND}_{2}$ & & & 2.96 & & \\
\hline $\mathrm{OCH}_{2}\left(\mathrm{CH}_{2}\right)_{5} \mathrm{ND}_{2}$ & & & $3.67,3.92$ & & \\
\hline $\mathrm{NDCOCH}_{3}$ & & & 2.03 & & \\
\hline
\end{tabular}

${ }^{\text {a }} \mathrm{Glc}\left(\beta 1-\mathrm{O}\left(\mathrm{CH}_{2}\right)_{6} \mathrm{NH}_{2}\right)$.

b $\mathrm{Gal}(\beta 1-4) \mathrm{Glc}$.

c $\operatorname{Glc}(\beta 1-6)$ GlcNAc.

d Gal( $\beta 1-4)$ GlcNAc.

e n.d., not determined.

anhydride in pyridine afforded 45 (86\% over three steps). O-De-acetylation of $\mathbf{4 5}$ using sodium methoxide $(\mathrm{pH} 10)(\rightarrow \mathbf{4 6}, 79 \%)$, and reduction of the azide function using $10 \% \mathrm{Pd}-\mathrm{C}$ and $\mathrm{H}_{2}$ in the presence of ammonia, gave the linear tetrasaccharide backbone $\mathbf{8}$ in $82 \%$ yield. Hexasaccharide 4 was synthesized in $76 \%$ yield by the transfer of galactose from UDP-galactose to $\mathrm{O}-4$ of the $N$-acetyl- $\beta$-D-glucosamine residues of 8 by using bovine milk $\beta$-1,4-galactosyltransferase as a catalyst (Scheme 9). ${ }^{1} \mathrm{H}$ NMR data of $\mathbf{4 6 , 8}, \mathbf{8}$, and $\mathbf{4}$, derived from 2D TOCSY and ROESY measurements, are presented in Tables $8-10$, respectively.

Conjugation of the oligosaccharides 1-4 to $\mathrm{CRM}_{197}$ (cross reactive material) and immunological studies are in progress.

\section{Experimental}

\subsection{General methods}

All chemicals were of reagent grade, and were used without further purification. Reactions were monitored by TLC on Silica Gel $60 \mathrm{~F}_{254}$ (E. Merck); after examination under UV light, compounds were visualized by heating with $10 \%(\mathrm{v} / \mathrm{v})$ ethanolic $\mathrm{H}_{2} \mathrm{SO}_{4}$, orcinol ( $2 \mathrm{mg} / \mathrm{mL}$ ) in $20 \%$ (v/v) methanolic $\mathrm{H}_{2} \mathrm{SO}_{4}$, or ninhydrin $(1.5 \mathrm{mg} / \mathrm{mL})$ in 38:1.75:0.25 1-BuOH-water-AcOH. In the work-up procedures of reaction mixtures, organic solutions were washed with appropriate amounts of the indicated aqueous solutions, then dried $\left(\mathrm{MgSO}_{4}\right.$ or $\mathrm{Na}_{2} \mathrm{SO}_{4}$ ), and concentrated under diminished pressure at $40{ }^{\circ} \mathrm{C}$. Column chromatography was performed on Silica Gel 60 (E. Merck, 0.063-0.200 mm). Optical rotations were measured with a Perkin-Elmer 241 polarimeter, using a $10 \mathrm{~cm}, 1 \mathrm{~mL}$ cell. ${ }^{1} \mathrm{H}$ NMR spectra were recorded at $300 \mathrm{~K}$ with a Bruker AC $300(300$ $\mathrm{MHz})$ or a Bruker AMX $500(500 \mathrm{MHz})$ spectrometer; the $\delta_{\mathrm{H}}$ values are given in ppm relative to the signal for internal $\mathrm{Me}_{4} \mathrm{Si}\left(\delta_{\mathrm{H}} 0, \mathrm{CDCl}_{3}\right)$ or internal acetone $\left(\delta_{\mathrm{H}}\right.$ 2.225, $\mathrm{D}_{2} \mathrm{O}$ ). ${ }^{13} \mathrm{C}$ NMR spectra (APT, $75.5 \mathrm{MHz}$ ) were recorded at $300 \mathrm{~K}$ with a Bruker AC 300 spectrometer; $\delta_{\mathrm{C}}$ values are given in $\mathrm{ppm}$ relative to the signal of $\mathrm{CDCl}_{3}\left(\delta_{\mathrm{C}} 76.9, \mathrm{CDCl}_{3}\right)$ or internal acetone $\left(\delta_{\mathrm{C}} 30.89\right.$, $\mathrm{D}_{2} \mathrm{O}$ ). Two-dimensional ${ }^{1} \mathrm{H}-{ }^{1} \mathrm{H}$ TOCSY (mixing times 7 and $100 \mathrm{~ms}$ ) and ROESY (mixing time $300 \mathrm{~ms}$ ), and ${ }^{1} \mathrm{H}-{ }^{13} \mathrm{C}$ correlated HSQC NMR spectra were recorded at $300 \mathrm{~K}$ with a Bruker AMX 500 spectrometer. Exact masses were measured by nano electrospray time-offlight mass spectrometry (positive-ion mode) using a Micromass LCToF mass spectrometer at a resolution of 5000 FWHM. Gold-coated capillaries were loaded with $1 \mu \mathrm{L}$ of sample (conc $20 \mu \mathrm{M}$ ) dissolved in $1: 1 \mathrm{MeCN}-$ water with $0.1 \%$ formic acid. Pentafluorophenylalanine was added as internal standard. The capillary voltage was set at $1500 \mathrm{~V}$ and the cone voltage was set at $30 \mathrm{~V}$.

\subsection{Allyl (2,3,4,6-tetra- $O$-acetyl- $\beta$-D-glucopyranosyl)- $(1 \rightarrow 6)-2-d e o x y-3,4-d i-O$ - $p$-methylbenzoyl-2- phthalimido- $\beta$-D-glucopyranoside (11)}

A soln of allyl 2-deoxy-3,4-di- $O$ - $p$-methylbenzoyl-2phthalimido- $\beta$-D-glucopyranoside $(\mathbf{1 0})^{11}(0.15 \mathrm{~g}, 0.26$ $\mathrm{mmol}$ ) and 2,3,4,6-tetra- $O$-acetyl- $\alpha$-D-glucopyranosyl 


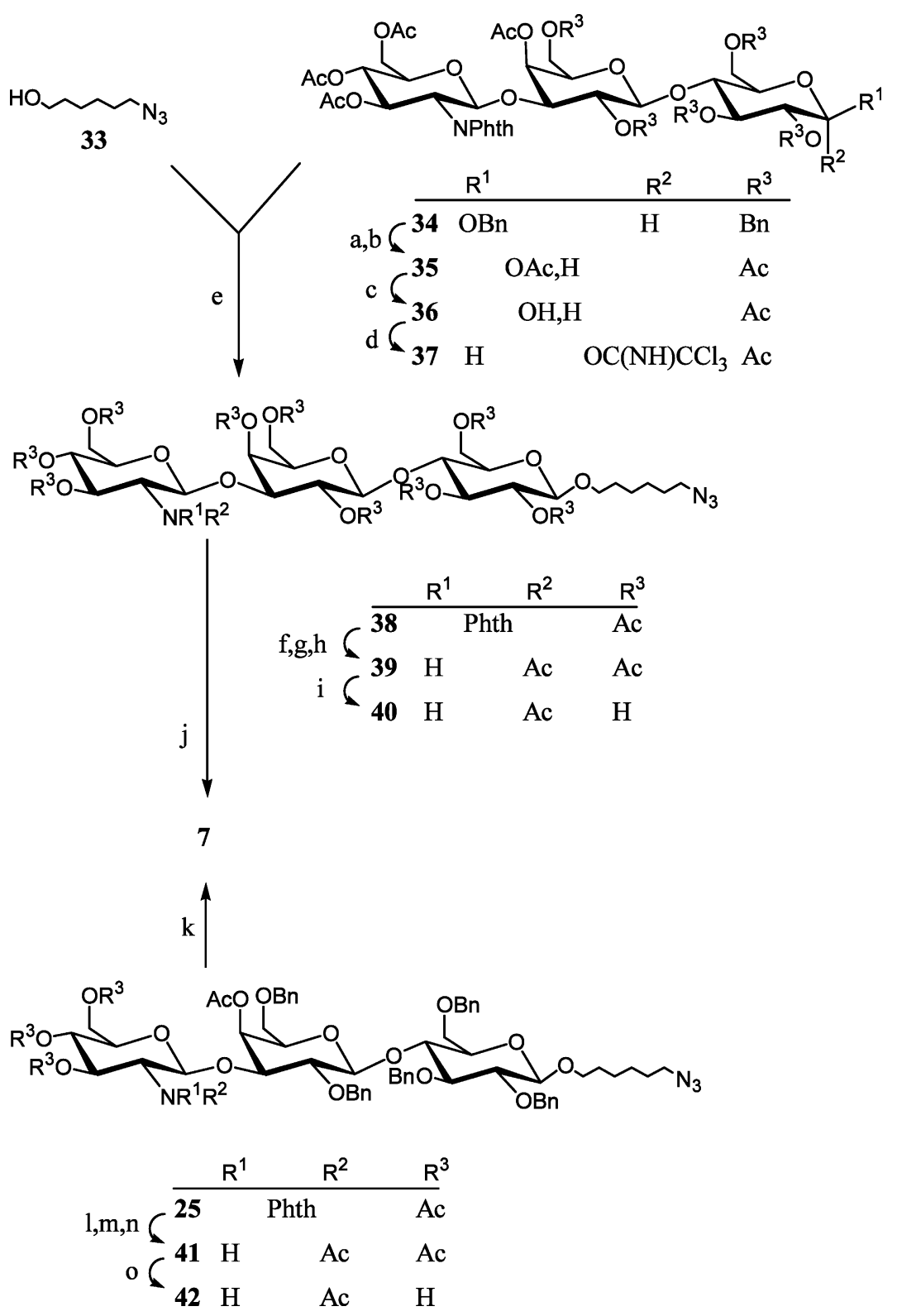

Scheme 6. Synthesis of the linear trisaccharide backbone 7: (a) 10\% Pd-C, $\mathrm{H}_{2}, \mathrm{EtOH}, \mathrm{EtOAc}, \mathrm{AcOH}$; (b) pyridine, Ac $\mathrm{C}_{2} \mathrm{O}, 66 \%$ over two steps; (c) hydrazinium acetate, DMF, $89 \%$; (d) $\mathrm{Cl}_{3} \mathrm{CCN}, \mathrm{DBU}, \mathrm{CH}_{2} \mathrm{Cl}_{2}, 69 \%$; (e) 1 equiv $\mathrm{AgOTf} \mathrm{CH}_{2} \mathrm{Cl}_{2}, 0{ }^{\circ} \mathrm{C}, 22 \%$; (f) $\mathrm{NaOMe}(\mathrm{pH} 10), \mathrm{MeOH}, \mathrm{CH}_{2} \mathrm{Cl}_{2}$; (g) $\mathrm{NH}_{2} \mathrm{CH}_{2} \mathrm{CH}_{2} \mathrm{NH}_{2}, 1-\mathrm{BuOH}, 80{ }^{\circ} \mathrm{C}$; (h) pyridine, $\mathrm{Ac}_{2} \mathrm{O}, 77 \%$ over three steps; (i) $\mathrm{NaOMe}$ (pH 10), $\mathrm{MeOH}, \mathrm{CH}_{2} \mathrm{Cl}_{2}, 55 \%$; (j) $10 \% \mathrm{Pd}-\mathrm{C}, \mathrm{H}_{2}$, water, tert $-\mathrm{BuOH}$, aq $25 \% \mathrm{NH}_{3}, 83 \%$; (k) $10 \% \mathrm{Pd}-\mathrm{C}, \mathrm{H}_{2}$, water, tert- $\mathrm{BuOH}$, aq $25 \%$ $\mathrm{NH}_{3} / 10 \% \mathrm{Pd}-\mathrm{C}, \mathrm{AcOH}, 68 \%$; (l) NaOMe (pH 10), $\mathrm{MeOH}, \mathrm{CH}_{2} \mathrm{Cl}_{2}$; (m) $\mathrm{NH}_{2} \mathrm{CH}_{2} \mathrm{CH}_{2} \mathrm{NH}_{2}, 1-\mathrm{BuOH}, 80{ }^{\circ} \mathrm{C}$; (n) pyridine, Ac $\mathrm{A}_{2} \mathrm{O}$, $78 \%$ over three steps; (o) $\mathrm{NaOMe}(\mathrm{pH} 10), \mathrm{MeOH}, \mathrm{CH}_{2} \mathrm{Cl}_{2}, 99 \%$.

trichloroacetimidate $(9)^{16}(0.16 \mathrm{~g}, 0.32 \mathrm{mmol})$ in dry $\mathrm{CH}_{2} \mathrm{Cl}_{2}(7.5 \mathrm{~mL})$, containing $4 \AA$ molecular sieves $(0.1$ g), was stirred under Ar for $0.5 \mathrm{~h}$. After cooling to $0{ }^{\circ} \mathrm{C}$, AgOTf $(77 \mathrm{mg}, 0.3 \mathrm{mmol}$ ) was added and the mixture was stirred for $1.5 \mathrm{~h}$. After filtration, the soln was washed with aq $10 \% \mathrm{NaHSO}_{3}$, aq satd $\mathrm{NaHCO}_{3}$, and water, dried $\left(\mathrm{MgSO}_{4}\right)$, filtered, and concentrated. Lowpressure column chromatography $(10: 1 \rightarrow 4: 1$ tolueneEtOAc) of the residue gave 11, isolated as a white foam $(0.14 \mathrm{~g}, 62 \%) ; R_{f} 0.65(1: 1$ toluene-EtOAc $) ;[\alpha]_{\mathrm{D}}^{20}-4^{\circ}(c$ $\left.1, \mathrm{CHCl}_{3}\right) ;{ }^{1} \mathrm{H}$ NMR $\left(500 \mathrm{MHz}, \mathrm{CDCl}_{3} ; 2 \mathrm{D}\right.$ TOCSY,
ROESY): $\delta 2.00,2.01$, and $2.08\left(3 \mathrm{~s}, 6,3,3 \mathrm{H}, 4 \mathrm{COCH}_{3}\right)$, 2.23 and $2.31\left(2 \mathrm{~s}\right.$, each $\left.3 \mathrm{H}, 2 \mathrm{COC}_{6} \mathrm{H}_{4} \mathrm{CH}_{3}\right), 3.71(\mathrm{~m}, 1$ $\left.\mathrm{H}, \mathrm{H}-5^{\mathrm{II}}\right), 3.83\left(\mathrm{dd}, 1 \mathrm{H}, J_{5,6 \mathrm{~b}} 7.9, J_{6 \mathrm{a}, 6 \mathrm{~b}} 10.8 \mathrm{~Hz}, \mathrm{H}-6 \mathrm{~b}^{\mathrm{I}}\right)$, $4.23\left(\mathrm{dd}, 1 \mathrm{H}, J_{5,6 \mathrm{~b}} 4.6, J_{6 \mathrm{a}, 6 \mathrm{~b}} 12.1 \mathrm{~Hz}, \mathrm{H}-6 \mathrm{~b}^{\mathrm{II}}\right), 4.36$ (m, 1 $\left.\mathrm{H}, \mathrm{OCH} H \mathrm{CH}=\mathrm{CH}_{2}\right), 4.54\left(\mathrm{dd}, 1 \mathrm{H}, J_{1,2} 8.4, J_{2,3} 10.5\right.$ $\left.\mathrm{Hz}, \mathrm{H}-2^{\mathrm{I}}\right), 4.69\left(\mathrm{~d}, 1 \mathrm{H}, J_{1,2} 7.9 \mathrm{~Hz}, \mathrm{H}-1^{1 \mathrm{II}}\right), 5.39(\mathrm{t}, 1 \mathrm{H}$, $\left.\mathrm{H}-4^{\mathrm{I}}\right), 5.57\left(\mathrm{~d}, 1 \mathrm{H}, \mathrm{H}-1^{\mathrm{I}}\right), 5.77\left(\mathrm{~m}, 1 \mathrm{H}, \mathrm{OCH}_{2} \mathrm{CH}=\right.$ $\left.\mathrm{CH}_{2}\right), 6.22\left(\mathrm{dd}, 1 \mathrm{H}, J_{3,4} 9.4 \mathrm{~Hz}, \mathrm{H}-3^{\mathrm{I}}\right), 7.02$ and 7.14 ( $2 \mathrm{~d}$, each $2 \mathrm{H}$, Phth), 7.61-7.80 (2 m, $8 \mathrm{H}, 2$ $\left.\mathrm{COC}_{6} \mathrm{H}_{4} \mathrm{CH}_{3}\right) ;{ }^{13} \mathrm{C} \mathrm{NMR}\left(75.5 \mathrm{MHz}, \mathrm{CDCl}_{3}\right): \delta 20.2-$ $20.4\left(\mathrm{COCH}_{3}\right), 21.2$ and $21.3\left(2 \mathrm{COC}_{6} \mathrm{H}_{4} \mathrm{CH}_{3}\right), 54.6(\mathrm{C}-$ 
Table 5

$500 \mathrm{MHz}{ }^{1} \mathrm{H}$ NMR data (TOCSY, ROESY) of 40 at $300 \mathrm{~K}$ (in ppm)

\begin{tabular}{|c|c|c|c|}
\hline \multirow[t]{2}{*}{ Proton } & \multicolumn{3}{|l|}{$\delta_{\mathrm{H}}$} \\
\hline & Glc I & Gal II & GlcNAc III \\
\hline $\mathrm{H}-1$ & 4.48 & 4.43 & 4.68 \\
\hline $\mathrm{H}-2$ & 3.29 & 3.60 & 3.76 \\
\hline $\mathrm{H}-3$ & 3.65 & 3.72 & 3.58 \\
\hline $\mathrm{H}-4$ & n.d. ${ }^{a}$ & 4.15 & 3.47 \\
\hline $\mathrm{H}-5$ & 3.60 & n.d. & 3.47 \\
\hline H-6a & 3.97 & n.d. & 3.89 \\
\hline $\mathrm{H}-6 \mathrm{~b}$ & 3.80 & n.d. & 3.76 \\
\hline $\mathrm{O}\left(\mathrm{CH}_{2}\right)_{2}\left(\mathrm{CH}_{2}\right)_{2}\left(\mathrm{CH}_{2}\right)_{2} \mathrm{~N}_{3}$ & & & $1.39-1.42(4 \mathrm{H})$ \\
\hline $\mathrm{OCH}_{2} \mathrm{CH}_{2}\left(\mathrm{CH}_{2}\right)_{2} \mathrm{CH}_{2} \mathrm{CH}_{2} \mathrm{~N}_{3}$ & & & $1.63-1.65(4 \mathrm{H})$ \\
\hline $\mathrm{CH}_{2} \mathrm{~N}_{3}$ & & & 3.32 \\
\hline $\mathrm{OCH}_{2}\left(\mathrm{CH}_{2}\right)_{5} \mathrm{~N}_{3}$ & & & $3.62,3.92$ \\
\hline $\mathrm{NDCOCH}_{3}$ & & & 2.04 \\
\hline
\end{tabular}

a n.d., not determined.

$\left.2^{\mathrm{I}}\right), 53.2,61.6$, and $69.8\left(\mathrm{C}-6^{\mathrm{I}}, \mathrm{C}-6^{\mathrm{II}}, \mathrm{OCH}_{2} \mathrm{CH}=\mathrm{CH}_{2}\right)$, $68.1,69.9,70.7,71.0,71.6,72.6$, and $73.6\left(\mathrm{C}-3^{\mathrm{I}}, \mathrm{C}-4^{\mathrm{I}}, \mathrm{C}-\right.$ $\left.5^{\mathrm{I}}, \mathrm{C}-2^{\mathrm{II}}, \mathrm{C}-3^{\mathrm{II}}, \mathrm{C}-4^{\mathrm{II}}, \mathrm{C}-5^{\mathrm{II}}\right), 96.9$ and $100.5\left(\mathrm{C}-1^{\mathrm{I}}, \mathrm{C}-1^{\mathrm{II}}\right)$, $117.5\left(\mathrm{OCH}_{2} \mathrm{CH}=\mathrm{CH}_{2}\right), \quad 165.0$ and 165.2 $\left.C \mathrm{OC}_{6} \mathrm{H}_{4} \mathrm{CH}_{3}\right), 168.9-170.2\left(\mathrm{COCH}_{3}\right)$; HRMS data of $\mathrm{C}_{47} \mathrm{H}_{49} \mathrm{NO}_{18}(\mathrm{M}, 915.295)$ : $\left[\mathrm{M}+\mathrm{NH}_{4}\right]^{+}$found 933.335, calcd 933.329.

Table 6

$500 \mathrm{MHz}{ }^{1} \mathrm{H}$ NMR data (TOCSY, ROESY) of 7 at $300 \mathrm{~K}$ (in ppm)

\begin{tabular}{|c|c|c|c|}
\hline \multirow[t]{2}{*}{ Proton } & \multicolumn{3}{|l|}{$\delta_{\mathrm{H}}$} \\
\hline & $\begin{array}{l}\text { Glc } \\
\text { I }\end{array}$ & Gal II & $\begin{array}{l}\text { GlcNAc } \\
\text { III }\end{array}$ \\
\hline H-1 & 4.47 & 4.43 & 4.68 \\
\hline $\mathrm{H}-2$ & 3.29 & 3.59 & 3.74 \\
\hline $\mathrm{H}-3$ & 3.64 & 3.73 & 3.56 \\
\hline $\mathrm{H}-4$ & 3.64 & 4.15 & 3.46 \\
\hline $\mathrm{H}-5$ & 3.59 & n.d. ${ }^{a}$ & 3.46 \\
\hline H-6a & 3.98 & n.d. & 3.90 \\
\hline $\mathrm{H}-6 \mathrm{~b}$ & 3.80 & n.d. & 3.77 \\
\hline $\mathrm{O}\left(\mathrm{CH}_{2}\right)_{2}\left(\mathrm{CH}_{2}\right)_{2}\left(\mathrm{CH}_{2}\right)_{2} \mathrm{ND}_{2}$ & & $\begin{array}{l}1.39-1.41 \\
(4 \mathrm{H})\end{array}$ & \\
\hline $\mathrm{OCH}_{2} \mathrm{CH}_{2}\left(\mathrm{CH}_{2}\right)_{2} \mathrm{CH}_{2} \mathrm{CH}_{2} \mathrm{ND}_{2}$ & & $\begin{array}{l}1.63-1.65 \\
(4 \mathrm{H})\end{array}$ & \\
\hline $\mathrm{CH}_{2} \mathrm{ND}_{2}$ & & 2.95 & \\
\hline $\mathrm{OCH}_{2}\left(\mathrm{CH}_{2}\right)_{5} \mathrm{ND}_{2}$ & & $3.58,3.83$ & \\
\hline $\mathrm{NDCOCH}_{3}$ & & 2.03 & \\
\hline
\end{tabular}

\footnotetext{
a n.d., not determined.
}

\section{3. $(2,3,4,6$-Tetra- $O$-acetyl- $\beta$-D-glucopyranosyl)-( $1 \rightarrow$ 6)-2-deoxy-3,4-di- $O$-p -methylbenzoyl-2-phthalimido- $\alpha, \beta$ - D-glucopyranose (12)}

To a soln of $\mathbf{1 1}(0.41 \mathrm{~g}, 0.45 \mathrm{mmol})$ in AcOH $(15 \mathrm{~mL})$ were added $\mathrm{Pd}(\mathrm{II}) \mathrm{Cl}_{2}(0.36 \mathrm{~g}, 2.03 \mathrm{mmol})$ and $\mathrm{NaOAc}$ $(0.31 \mathrm{~g}, 3.78 \mathrm{mmol})$, and the mixture was kept overnight in an ultrasonic bath. After filtration over hyflo, the soln was diluted with $\mathrm{CH}_{2} \mathrm{Cl}_{2}$ then washed with water, aq satd $\mathrm{NaHCO}_{3}$, and water, dried $\left(\mathrm{MgSO}_{4}\right)$, filtered, and concentrated. Low-pressure column chromatography $(4: 1 \rightarrow 1: 1$ toluene-EtOAc) of the residue gave 12, isolated as a slightly yellow foam $(0.27 \mathrm{~g}, 70 \%) ; R_{f}$ 0.45 (1:1 toluene-EtOAc); ${ }^{1} \mathrm{H}$ NMR $\beta$-product (300 $\left.\mathrm{MHz}, \mathrm{CDCl}_{3}\right): \delta 2.02,2.04,2.05$, and $2.12(4 \mathrm{~s}$, each 3 $\left.\mathrm{H}, 4 \mathrm{COCH}_{3}\right), 2.27$ and $2.34(2 \mathrm{~s}$, each $3 \mathrm{H}, 2$ $\left.\mathrm{COC}_{6} \mathrm{H}_{4} \mathrm{CH}_{3}\right), 4.02\left(\mathrm{dd}, 1 \mathrm{H}, J_{5,6 \mathrm{a}} 1.9, J_{6 \mathrm{a}, 6 \mathrm{~b}} 11.2 \mathrm{~Hz}\right.$, $\left.\mathrm{H}-6 \mathrm{a}^{\mathrm{I}}\right), 4.20\left(\mathrm{dd}, 1 \mathrm{H}, J_{5,6 \mathrm{~b}} 4.7, J_{6 \mathrm{a}, 6 \mathrm{~b}} 12.2 \mathrm{~Hz}, \mathrm{H}-6 \mathrm{~b}^{\mathrm{II}}\right)$, $4.43\left(\mathrm{dd}, 1 \mathrm{H}, J_{1,2} 8.4, J_{2,3} 10.7 \mathrm{~Hz}, \mathrm{H}-2^{\mathrm{I}}\right), 4.65(\mathrm{~d}, 1 \mathrm{H}$, $\left.J_{1,2} 7.5 \mathrm{~Hz}, \mathrm{H}-1^{\mathrm{II}}\right), 4.96\left(\mathrm{dd}, 1 \mathrm{H}, J_{2,3} 8.6 \mathrm{~Hz}, \mathrm{H}-2^{\mathrm{II}}\right), 5.42$ $\left(\mathrm{t}, 1 \mathrm{H}, \mathrm{H}-4^{\mathrm{I}}\right), 5.74\left(\mathrm{~d}, 1 \mathrm{H}, \mathrm{H}-1^{\mathrm{I}}\right), 6.23\left(\mathrm{dd}, 1 \mathrm{H}, J_{3,4} 9.2\right.$ $\left.\mathrm{Hz}, \mathrm{H}-3^{\mathrm{I}}\right), 7.04$ and 7.15 ( $2 \mathrm{~d}$, each $\left.2 \mathrm{H}, \mathrm{Phth}\right), 7.60-7.81$ $\left(2 \mathrm{~m}, 8 \mathrm{H}, 2 \mathrm{COC}_{6} \mathrm{H}_{4} \mathrm{CH}_{3}\right) ;{ }^{13} \mathrm{C}$ NMR $(75.5 \mathrm{MHz}$, $\left.\mathrm{CDCl}_{3}\right): \delta$ 20.3-20.4 $\left(\mathrm{COCH}_{3}\right), 21.2$ and 21.3 (2 $\left.\mathrm{COC}_{6} \mathrm{H}_{4} \mathrm{CH}_{3}\right), 56.1\left(\mathrm{C}-2^{\mathrm{I}}\right), 61.7$ and $68.3\left(\mathrm{C}-6^{\mathrm{I}}, \mathrm{C}-6^{\mathrm{II}}\right)$, 68.1, 69.7, 70.7, 71.2, 71.5, 72.5, and $73.3\left(\mathrm{C}-3^{\mathrm{I}}, \mathrm{C}-4^{\mathrm{I}}, \mathrm{C}-\right.$ $\left.5^{\mathrm{I}}, \mathrm{C}-2^{\mathrm{II}}, \mathrm{C}-3^{\mathrm{II}}, \mathrm{C}-4^{\mathrm{II}}, \mathrm{C}-5^{\mathrm{II}}\right), 92.4$ and $100.5\left(\mathrm{C}-1^{\mathrm{I}}, \mathrm{C}-1^{\mathrm{II}}\right)$, 165.0 and $165.2 \quad\left(2 \quad \mathrm{COC}_{6} \mathrm{H}_{4} \mathrm{CH}_{3}\right), \quad 169.2-170.4$ $\left(\mathrm{COCH}_{3}\right)$; HRMS of $\mathrm{C}_{44} \mathrm{H}_{45} \mathrm{NO}_{18}(\mathrm{M}, 875.263)$ : [M+ $\mathrm{H}]^{+}$found 876.273 , calcd 876.271 .

\section{4. $(2,3,4,6$-Tetra- $O$-acetyl- $\beta$-D-glucopyranosyl)-( $1 \rightarrow$ 6)-2-deoxy-3,4-di- $O$ - $p$-methylbenzoyl-2-phthalimido- $\alpha, \beta$ - D-glucopyranosyl trichloroacetimidate (13)}

To a soln of $12(0.37 \mathrm{~g}, 0.42 \mathrm{mmol})$ in dry $\mathrm{CH}_{2} \mathrm{Cl}_{2}(15$ $\mathrm{mL})$ were added, at $0{ }^{\circ} \mathrm{C}$, trichloroacetonitrile $(0.2 \mathrm{~mL}$, $2.1 \mathrm{mmol})$ and 1,8-diazabicyclo[5.4.0]undec-7-ene (60 $\mu \mathrm{L}, 0.041 \mathrm{mmol}$ ), and the mixture was stirred for $1.5 \mathrm{~h}$, then concentrated. Column chromatography (2:1 toluene-EtOAc) of the residue gave 13, isolated as a slightly yellow foam $(0.33 \mathrm{~g}, 76 \%) ; R_{f} 0.64$ (1:1 tolueneEtOAc); ${ }^{1} \mathrm{H}$ NMR $\beta$-product $\left(300 \mathrm{MHz}, \mathrm{CDCl}_{3}\right): \delta$ $1.99,2.03$, and $2.09\left(3 \mathrm{~s}, 6,3,3 \mathrm{H}, 4 \mathrm{COCH}_{3}\right), 2.27$ and $2.34\left(2 \mathrm{~s}\right.$, each $\left.3 \mathrm{H}, 2 \mathrm{COC}_{6} \mathrm{H}_{4} \mathrm{CH}_{3}\right), 3.62(\mathrm{~m}, 1 \mathrm{H}, \mathrm{H}-$ $\left.5^{\mathrm{II}}\right), 3.86\left(\mathrm{dd}, 1 \mathrm{H}, J_{5,6 \mathrm{~b}} 6.8, J_{6 \mathrm{a}, 6 \mathrm{~b}} 11.8 \mathrm{~Hz}, \mathrm{H}-6 \mathrm{~b}^{\mathrm{I}}\right), 4.70$ $\left(\mathrm{d}, 1 \mathrm{H}, J_{1,2} 7.8 \mathrm{~Hz}, \mathrm{H}-1^{\mathrm{II}}\right), 5.15\left(\mathrm{t}, 1 \mathrm{H}, \mathrm{H}-3^{\mathrm{II}}\right), 5.50(\mathrm{t}, 1$ $\left.\mathrm{H}, \mathrm{H}-4^{\mathrm{I}}\right), 6.32\left(\mathrm{t}, 1 \mathrm{H}, \mathrm{H}-3^{\mathrm{I}}\right), 6.79\left(\mathrm{~d}, 1 \mathrm{H}, J_{1,2} 8.8 \mathrm{~Hz}, \mathrm{H}-\right.$ $\left.1^{\mathrm{I}}\right), 7.05$ and $7.15(2 \mathrm{~d}$, each $2 \mathrm{H}$, Phth $), 7.63-7.81(2 \mathrm{~m}$, $\left.8 \mathrm{H}, 2 \mathrm{COC}_{6} \mathrm{H}_{4} \mathrm{CH}_{3}\right), 8.78(\mathrm{~s}, 1 \mathrm{H}, \mathrm{NH}) ;{ }^{13} \mathrm{C} \mathrm{NMR}(75.5$ $\left.\mathrm{MHz}, \mathrm{CDCl}_{3}\right): \delta 20.4-20.5\left(\mathrm{COCH}_{3}\right), 21.3$ and $21.4(2$ $\left.\mathrm{COC}_{6} \mathrm{H}_{4} \mathrm{CH}_{3}\right), 53.7\left(\mathrm{C}-2^{\mathrm{I}}\right), 61.7$ and $67.3\left(\mathrm{C}-6^{\mathrm{I}}, \mathrm{C}-6^{\mathrm{II}}\right)$, $68.2,69.3,70.5,70.9,71.6,72.7$, and $75.0\left(\mathrm{C}-3^{\mathrm{I}}, \mathrm{C}-4^{\mathrm{I}}, \mathrm{C}-\right.$ $\left.5^{\mathrm{I}}, \mathrm{C}-2^{\mathrm{II}}, \mathrm{C}-3^{\mathrm{II}}, \mathrm{C}-4^{\mathrm{II}}, \mathrm{C}-5^{\mathrm{II}}\right), 93.4$ and $100.2\left(\mathrm{C}^{-1}{ }^{\mathrm{I}}, \mathrm{C}-1^{\mathrm{II}}\right)$, $160.2\left(\mathrm{OC}(\mathrm{NH}) \mathrm{CCl}_{3}\right), \quad 165.0$ and $165.3 \quad$ (2 $\left.\mathrm{COC}_{6} \mathrm{H}_{4} \mathrm{CH}_{3}\right), 169.1-170.3\left(\mathrm{COCH}_{3}\right)$. 


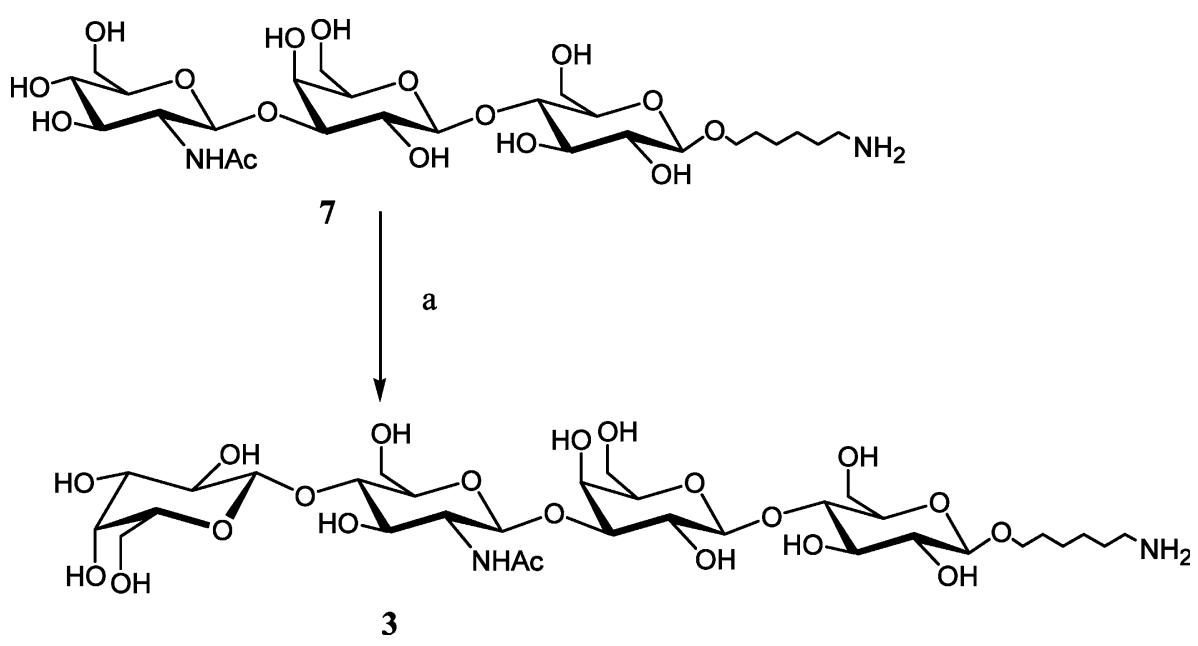

Scheme 7. Synthesis of tetrasaccharide 3: (a) 1.5 equiv UDP-Gal, aq $50 \mathrm{mM}$ sodium cacodylate buffer (pH 7.5), 2.5 U $\beta-1,4-$ galactosyltransferase, $14 \mathrm{U}$ alkaline phosphatase, $37^{\circ} \mathrm{C}, 94 \%$.

\subsection{6-Azidohexyl (3,4,6-tri- $O$-acetyl-2-deoxy-2- phthalimido- $\beta$-D-glucopyranosyl)-(1 $\rightarrow 3)-4-O$-acetyl-2,6- di- $O$-benzyl- $\beta$-D-galactopyranoside (16)}

A soln of 3,4,6-tri- $O$-acetyl-2-deoxy-2-phthalimido- $\beta$-Dglucopyranosyl trichloroacetimidate $(\mathbf{1 5})^{17}(1.09 \mathrm{~g}, 1.88$ mmol) and 6-azidohexyl 4- $O$-acetyl-2,6-di- $O$-benzyl- $\beta$ D-galactopyranoside $(\mathbf{1 4})^{14}(0.84 \mathrm{~g}, 1.47 \mathrm{mmol})$ in dry $\mathrm{CH}_{2} \mathrm{Cl}_{2}(50 \mathrm{~mL})$, containing $4 \AA$ molecular sieves $(1 \mathrm{~g})$, was stirred under Ar for $1 \mathrm{~h}$. After cooling to $-70^{\circ} \mathrm{C}$, TMSOTf $(34 \mu \mathrm{L}, 0.19 \mathrm{mmol})$ was added and the mixture was stirred for $2 \mathrm{~h}$, during which period the temperature was allowed to reach room temperature (rt). The mixture was neutralized with $\mathrm{Et}_{3} \mathrm{~N}$, filtered, washed with water, dried $\left(\mathrm{MgSO}_{4}\right)$, filtered, and concentrated.
Low-pressure column chromatography $(10: 1 \rightarrow 4: 1$ toluene-EtOAc) of the residue gave 16, isolated as a white foam $(1.28 \mathrm{~g}, 86 \%) ; R_{f} 0.44$ (2:1 toluene-EtOAc); $[\alpha]_{\mathrm{D}}^{20}+3^{\circ}\left(c 1, \mathrm{CHCl}_{3}\right) ;{ }^{1} \mathrm{H} \mathrm{NMR}\left(300 \mathrm{MHz}, \mathrm{CDCl}_{3}\right): \delta$ $1.22-1.24\left(\mathrm{~m}, 4 \mathrm{H}, 2 \mathrm{CH}_{2}\right), 1.37-1.42\left(\mathrm{~m}, 2 \mathrm{H}, \mathrm{CH}_{2}\right)$, $1.48-1.52\left(\mathrm{~m}, 2 \mathrm{H}, \mathrm{CH}_{2}\right), 1.82,2.00,2.03$, and $2.08(4 \mathrm{~s}$, each $\left.3 \mathrm{H}, 4 \mathrm{COCH}_{3}\right), 3.07\left(\mathrm{t}, 2 \mathrm{H}, \mathrm{CH}_{2} \mathrm{~N}_{3}\right), 3.84(\mathrm{~m}, 1 \mathrm{H}$, $\mathrm{OCH} H), 4.48\left(\mathrm{~d}, 2 \mathrm{H}, \mathrm{OCH}_{2} \mathrm{C}_{6} \mathrm{H}_{5}\right), 4.56(\mathrm{~d}, 1 \mathrm{H}$, OCH $\left.\mathrm{HC}_{6} \mathrm{H}_{5}\right), 5.16\left(\mathrm{t}, 1 \mathrm{H}, \mathrm{H}-4^{\mathrm{II}}\right), 5.38\left(\mathrm{~d}, 1 \mathrm{H}, J_{3,4} 3.5\right.$, $\left.J_{4,5}<1 \mathrm{~Hz}, \mathrm{H}-4^{\mathrm{I}}\right), 5.59\left(\mathrm{~d}, 1 \mathrm{H}, J_{1,2} 8.3 \mathrm{~Hz}, \mathrm{H}-1^{\mathrm{II}}\right), 5.77$ (dd, $\left.1 \mathrm{H}, J_{2,3} 10.7, J_{3,4} 9.1 \mathrm{~Hz}, \mathrm{H}-3{ }^{\mathrm{II}}\right) ;{ }^{13} \mathrm{C}$ NMR $(75.5$ $\left.\mathrm{MHz}, \mathrm{CDCl}_{3}\right): \delta$ 20.2-20.5 $\left(\mathrm{COCH}_{3}\right), 25.3,26.2,28.4$, and $29.2\left(4 \mathrm{CH}_{2}\right), 51.0\left(\mathrm{CH}_{2} \mathrm{~N}_{3}\right), 54.7\left(\mathrm{C}-2^{\mathrm{II}}\right), 61.2,68.9$, 69.8, 73.4, and $73.9\left(\mathrm{C}-6{ }^{\mathrm{I}}, \mathrm{C}-6^{\mathrm{II}}, 2 \mathrm{OCH}_{2} \mathrm{C}_{6} \mathrm{H}_{5}, \mathrm{OCH}_{2}\right)$, 68.6, 69.5, 70.4, 71.6, 72.6, 78.1, and $78.5\left(\mathrm{C}-2^{\mathrm{I}}, \mathrm{C}-3^{\mathrm{I}}, \mathrm{C}-\right.$

Table 7

$500 \mathrm{MHz}{ }^{1} \mathrm{H}$ NMR data (TOCSY, ROESY) of 3 at $300 \mathrm{~K}$ (in ppm)

\begin{tabular}{|c|c|c|c|c|}
\hline \multirow[t]{2}{*}{ Proton } & \multicolumn{4}{|l|}{$\delta_{\mathrm{H}}$} \\
\hline & Glc I & Gal II $^{\mathrm{a}}$ & GlcNAc III & Gal IV $^{b}$ \\
\hline $\mathrm{H}-1$ & 4.48 & 4.44 & 4.71 & 4.48 \\
\hline $\mathrm{H}-2$ & 3.29 & 3.58 & 3.78 & 3.54 \\
\hline $\mathrm{H}-3$ & 3.63 & 3.72 & 3.73 & 3.67 \\
\hline $\mathrm{H}-4$ & 3.60 & 4.15 & 3.73 & 3.93 \\
\hline $\mathrm{H}-5$ & 3.62 & n.d. ${ }^{c}$ & 3.58 & n.d. \\
\hline H-6a & 3.96 & n.d. & 3.93 & n.d. \\
\hline $\mathrm{H}-6 \mathrm{~b}$ & 3.78 & n.d. & 3.85 & n.d. \\
\hline $\mathrm{O}\left(\mathrm{CH}_{2}\right)_{2}\left(\mathrm{CH}_{2}\right)_{2}\left(\mathrm{CH}_{2}\right)_{2} \mathrm{ND}_{2}$ & & \multicolumn{3}{|l|}{$1.39-1.42(4 \mathrm{H})$} \\
\hline $\mathrm{OCH}_{2} \mathrm{CH}_{2}\left(\mathrm{CH}_{2}\right)_{2} \mathrm{CH}_{2} \mathrm{CH}_{2} \mathrm{ND}_{2}$ & & \multicolumn{3}{|l|}{$1.63-1.70(4 \mathrm{H})$} \\
\hline $\mathrm{CH}_{2} \mathrm{ND}_{2}$ & & \multicolumn{3}{|l|}{2.99} \\
\hline $\mathrm{OCH}_{2}\left(\mathrm{CH}_{2}\right)_{5} \mathrm{ND}_{2}$ & & \multicolumn{3}{|l|}{$3.68,3.92$} \\
\hline $\mathrm{NDCOCH}_{3}$ & & \multicolumn{3}{|l|}{2.03} \\
\hline
\end{tabular}

a $\operatorname{Gal}(\beta 1-4)$ Glc.

b Gal( $\beta 1-4)$ GlcNAc.

c n.d., not determined. 


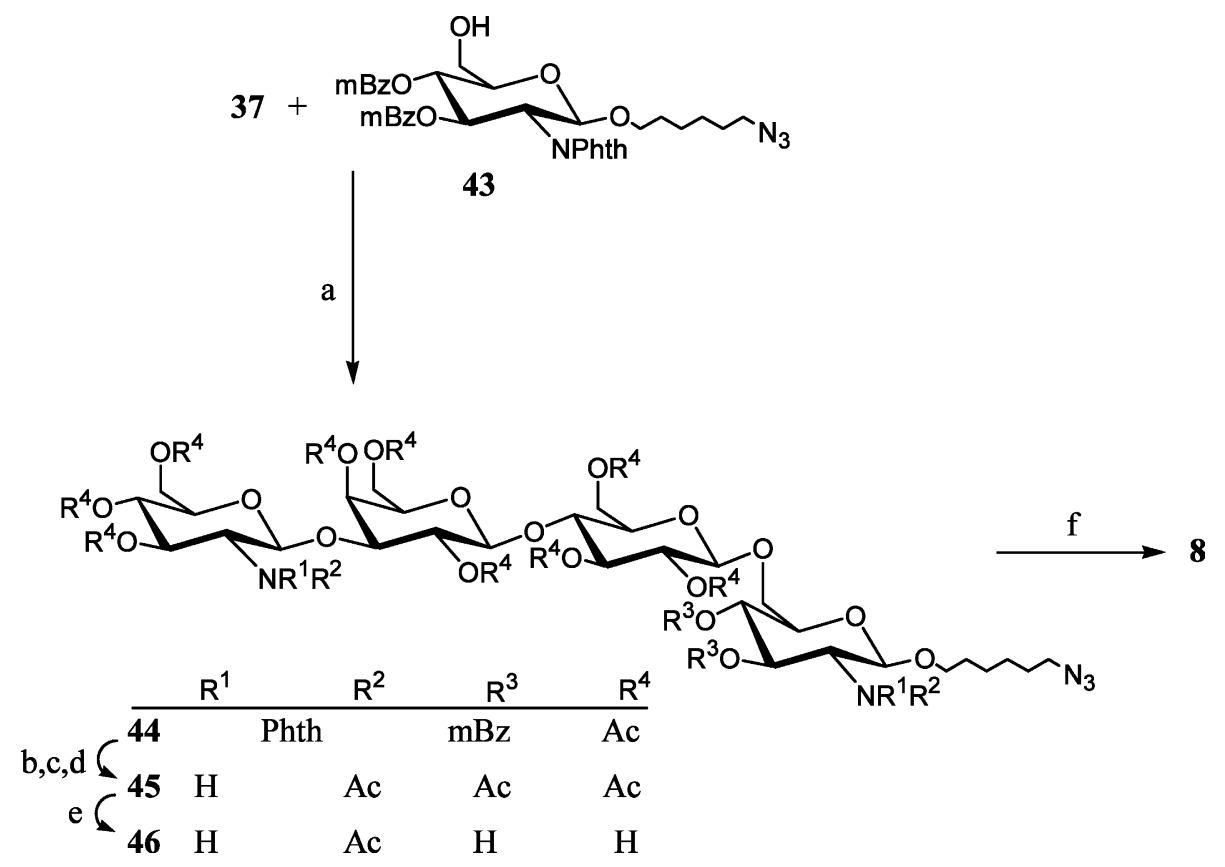

Scheme 8. Synthesis of the linear tetrasaccharide backbone 8: (a) 15\% TMSOTf, $\mathrm{CH}_{2} \mathrm{Cl}_{2}, 0{ }^{\circ} \mathrm{C}, 34 \%$; (b) $\mathrm{NaOMe}$ (pH 10), $\mathrm{MeOH}$, $\mathrm{CH}_{2} \mathrm{Cl}_{2}$; (c) $\mathrm{NH}_{2} \mathrm{CH}_{2} \mathrm{CH}_{2} \mathrm{NH}_{2}, 1-\mathrm{BuOH}, 90{ }^{\circ} \mathrm{C}$; (d) pyridine, $\mathrm{Ac}_{2} \mathrm{O}, 86 \%$ over three steps; (e) $\mathrm{NaOMe}(\mathrm{pH} \mathrm{10}), \mathrm{MeOH} \mathrm{CH}_{2} \mathrm{Cl}_{2}$, $79 \%$; (f) $10 \% \mathrm{Pd}-\mathrm{C}, \mathrm{H}_{2}$, water, tert $-\mathrm{BuOH}$, aq $25 \% \mathrm{NH}_{3}, 82 \%$.

$\left.4^{\mathrm{I}}, \mathrm{C}-5^{\mathrm{I}}, \mathrm{C}-3^{\mathrm{II}}, \mathrm{C}-4^{\mathrm{II}}, \mathrm{C}-5^{\mathrm{II}}\right), 97.9$ and $103.3\left(\mathrm{C}-1^{\mathrm{I}}, \mathrm{C}-1^{\mathrm{II}}\right)$, 169.1-170.4 $\left(\mathrm{COCH}_{3}\right)$; HRMS of $\mathrm{C}_{48} \mathrm{H}_{56} \mathrm{~N}_{4} \mathrm{O}_{16}(\mathrm{M}$, 944.369): $\left[\mathrm{M}+\mathrm{NH}_{4}\right]^{+}$found 962.399, calcd 962.403.

\subsection{6-Azidohexyl (2-deoxy-2-phthalimido- $\beta$-D-} glucopyranosyl)-( $(\rightarrow 3)-4-O$-acetyl-2,6-di- $O$-benzyl- $\beta$-Dgalactopyranoside (17)

To a soln of $16(1.28 \mathrm{~g}, 1.35 \mathrm{mmol})$ in $\mathrm{CH}_{2} \mathrm{Cl}_{2}(5 \mathrm{~mL})$ and $\mathrm{MeOH}(2 \mathrm{~mL})$ was added $\mathrm{NaOMe}(\mathrm{pH})$. The mixture was stirred for $3 \mathrm{~h}$, then neutralized with Dowex $50 \times 8\left(\mathrm{H}^{+}\right)$, filtered, and concentrated. Column chromatography (1:2 toluene-EtOAc) of the residue gave 17, isolated as a colourless glass $(1.01 \mathrm{~g}, 91 \%) ; R_{f} 0.36$ (1:3 toluene-EtOAc); $[\alpha]_{\mathrm{D}}^{20}+5^{\circ}\left(c 1, \mathrm{CHCl}_{3}\right) ;{ }^{1} \mathrm{H} \mathrm{NMR}$ (500 $\mathrm{MHz}, \mathrm{CDCl}_{3} ; 2 \mathrm{D}$ TOCSY, ROESY): $\delta$ 1.13-1.17 (m, $\left.4 \mathrm{H}, 2 \mathrm{CH}_{2}\right), 1.31-1.37\left(\mathrm{~m}, 2 \mathrm{H}, \mathrm{CH}_{2}\right), 1.39-1.44$ $\left(\mathrm{m}, 2 \mathrm{H}, \mathrm{CH}_{2}\right), 2.06\left(\mathrm{COCH}_{3}\right), 3.06\left(\mathrm{t}, 2 \mathrm{H}, \mathrm{CH}_{2} \mathrm{~N}_{3}\right)$, $4.00\left(\mathrm{dd}, 1 \mathrm{H}, J_{1,2} 8.5, J_{2,3} 10.5 \mathrm{~Hz}, \mathrm{H}-2^{\mathrm{II}}\right), 4.21(\mathrm{t}, 1 \mathrm{H}$, $\left.\mathrm{H}-3^{\mathrm{II}}\right), 4.24\left(\mathrm{~d}, 1 \mathrm{H}, J_{1,2} 7.7 \mathrm{~Hz}, \mathrm{H}-1^{\mathrm{I}}\right), 4.10$ and $4.41(2$

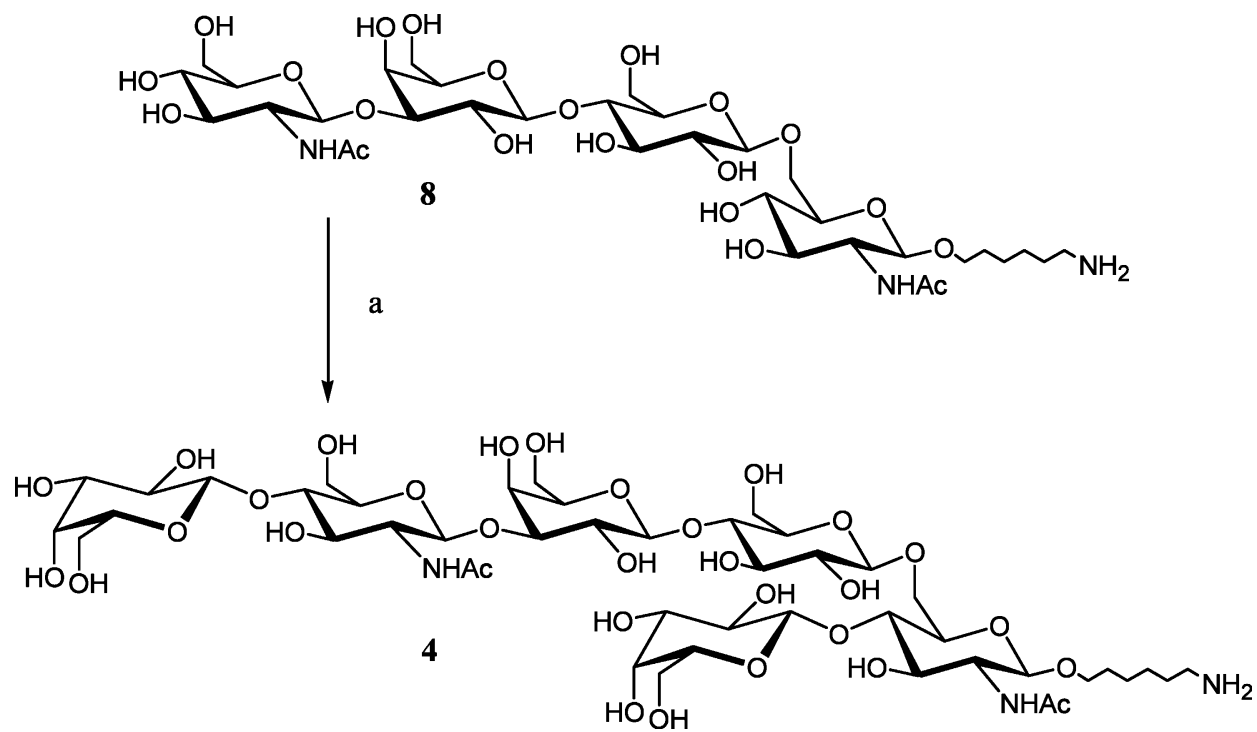

Scheme 9. Synthesis of hexasaccharide 4: (a) 3.2 equiv UDP-Gal, aq $50 \mathrm{mM}$ sodium cacodylate buffer (pH 7.5), 5 U $\beta-1,4-$ galactosyltransferase, $30 \mathrm{U}$ alkaline phosphatase, $37^{\circ} \mathrm{C}, 76 \%$. 
Table 8

$500 \mathrm{MHz}{ }^{1} \mathrm{H}$ NMR data (TOCSY, ROESY) of 46 at $300 \mathrm{~K}$ (in ppm)

\begin{tabular}{|c|c|c|c|c|}
\hline \multirow[t]{2}{*}{ Proton } & \multicolumn{4}{|l|}{$\delta_{\mathrm{H}}$} \\
\hline & GlcNAc I $^{\mathrm{a}}$ & Glc II & Gal III & GlcNAc IV $^{b}$ \\
\hline $\mathrm{H}-1$ & 4.52 & 4.56 & 4.44 & 4.69 \\
\hline H-2 & 3.69 & 3.38 & 3.59 & 3.56 \\
\hline $\mathrm{H}-3$ & 3.54 & 3.65 & 3.72 & 3.59 \\
\hline $\mathrm{H}-4$ & n.d. ${ }^{\mathrm{c}}$ & 3.65 & 4.16 & n.d. \\
\hline H-5 & 3.62 & 3.62 & n.d. & 3.48 \\
\hline $\mathrm{H}-6 \mathrm{a}$ & 4.22 & 3.99 & n.d. & 3.90 \\
\hline $\mathrm{H}-6 \mathrm{~b}$ & 3.89 & 3.81 & n.d. & 3.77 \\
\hline $\mathrm{O}\left(\mathrm{CH}_{2}\right)_{2}\left(\mathrm{CH}_{2}\right)_{2}\left(\mathrm{CH}_{2}\right)_{2} \mathrm{~N}_{3}$ & & & $1.35-1.37(4 \mathrm{H})$ & \\
\hline $\mathrm{OCH}_{2} \mathrm{CH}_{2}\left(\mathrm{CH}_{2}\right)_{2} \mathrm{CH}_{2} \mathrm{CH}_{2} \mathrm{~N}_{3}$ & & & $1.57-1.61(4 \mathrm{H})$ & \\
\hline $\mathrm{CH}_{2} \mathrm{~N}_{3}$ & & & 3.33 & \\
\hline $\mathrm{OCH}_{2}\left(\mathrm{CH}_{2}\right)_{5} \mathrm{~N}_{3}$ & & & $3.62,3.91$ & \\
\hline $\mathrm{NDCOCH}_{3}$ & & & $2.04(2)$ & \\
\hline
\end{tabular}

${ }^{\text {a }} \operatorname{GlcNAc}\left(\beta 1-\mathrm{O}\left(\mathrm{CH}_{2}\right)_{6} \mathrm{~N}_{3}\right)$.

b $\operatorname{GlcNAc}(\beta 1-3) \mathrm{Gal}$.

c n.d., not determined.

$\mathrm{d}$, each $\left.1 \mathrm{H}, \mathrm{OCH}_{2} \mathrm{C}_{6} \mathrm{H}_{5}\right), 4.46\left(\mathrm{~s}, 2 \mathrm{H}, \mathrm{OCH}_{2} \mathrm{C}_{6} \mathrm{H}_{5}\right)$, $5.36\left(\mathrm{~d}, 1 \mathrm{H}, \mathrm{H}-1^{\mathrm{II}}\right), 5.49\left(\mathrm{~d}, 1 \mathrm{H}, J_{3,4} 3.3, J_{4,5}<1 \mathrm{~Hz}, \mathrm{H}-\right.$ $\left.4^{\mathrm{I}}\right) ;{ }^{13} \mathrm{C}$ NMR $\left(75.5 \mathrm{MHz}, \mathrm{CDCl}_{3}\right): \delta 20.9\left(\mathrm{COCH}_{3}\right)$, 25.2, 26.1, 28.3, and $29.1\left(4 \mathrm{CH}_{2}\right), 51.1\left(\mathrm{CH}_{2} \mathrm{~N}_{3}\right), 56.6$ $\left(\mathrm{C}-2^{\mathrm{II}}\right), 61.3,68.7,69.8,73.4$, and $73.8\left(\mathrm{C}-6^{\mathrm{I}}, \mathrm{C}-6^{\mathrm{II}}, 2\right.$ $\left.\mathrm{OCH}_{2} \mathrm{C}_{6} \mathrm{H}_{5}, \mathrm{OCH}_{2}\right), 70.4,70.7,71.0,72.1,75.8,77.7$, and $79.9\left(\mathrm{C}-2^{\mathrm{I}}, \mathrm{C}-3^{\mathrm{I}}, \mathrm{C}-4^{\mathrm{I}}, \mathrm{C}-5^{\mathrm{I}}, \mathrm{C}-3^{\mathrm{II}}, \mathrm{C}-4^{\mathrm{II}}, \mathrm{C}-5^{\mathrm{II}}\right), 99.2$ and $103.3\left(\mathrm{C}-1^{\mathrm{I}}, \mathrm{C}-1^{\mathrm{II}}\right), 171.4\left(\mathrm{COCH}_{3}\right)$; HRMS of $\mathrm{C}_{42} \mathrm{H}_{50} \mathrm{~N}_{4} \mathrm{O}_{13}$ (M, 818.337): $[\mathrm{M}+\mathrm{Na}]^{+}$found 841.332, calcd 841.327.
3.7. 6-Azidohexyl (6-O-tert-butyldiphenylsilyl-2-deoxy2-phthalimido- $\beta$-D-glucopyranosyl)-( $1 \rightarrow 3)-4-O$-acetyl2,6-di- $O$-benzyl- $\beta$-D-galactopyranoside (18)

To a soln of $17(0.18 \mathrm{~g}, 0.23 \mathrm{mmol})$ in $\mathrm{CH}_{2} \mathrm{Cl}_{2}(5 \mathrm{~mL})$ and $\mathrm{Py}(0.4 \mathrm{~mL})$ were added 4-dimethylaminopyridine (20 mg, $0.18 \mathrm{mmol}), \mathrm{Et}_{3} \mathrm{~N}(70 \mu \mathrm{L})$, and tert-butyldiphenylsilyl chloride $(70 \mu \mathrm{L}, 0.27 \mathrm{mmol})$. The mixture was stirred for $4 \mathrm{~h}$, then poured into ice water, extracted with $\mathrm{CH}_{2} \mathrm{Cl}_{2}$, washed with aq satd $\mathrm{NaHCO}_{3}$, dried $\left(\mathrm{MgSO}_{4}\right)$,

Table 9

$500 \mathrm{MHz}{ }^{1} \mathrm{H}$ NMR data (TOCSY, ROESY) of 8 at $300 \mathrm{~K}$ (in ppm)

\begin{tabular}{|c|c|c|c|c|}
\hline \multirow[t]{2}{*}{ Proton } & \multicolumn{4}{|l|}{$\delta_{\mathrm{H}}$} \\
\hline & GlcNAc I $^{\mathrm{a}}$ & Glc II & Gal III & GlcNAc IV $^{\mathrm{b}}$ \\
\hline $\mathrm{H}-1$ & 4.49 & 4.54 & 4.44 & 4.69 \\
\hline $\mathrm{H}-2$ & 3.68 & 3.38 & 3.60 & 3.76 \\
\hline $\mathrm{H}-3$ & 3.53 & 3.66 & 3.72 & 3.57 \\
\hline $\mathrm{H}-4$ & 3.52 & 3.66 & 4.15 & 3.46 \\
\hline $\mathrm{H}-5$ & 3.60 & 3.59 & n.d. ${ }^{\mathrm{c}}$ & 3.46 \\
\hline H-6a & 4.21 & 3.97 & n.d. & 3.90 \\
\hline $\mathrm{H}-6 \mathrm{~b}$ & 3.88 & 3.81 & n.d. & 3.77 \\
\hline $\mathrm{O}\left(\mathrm{CH}_{2}\right)_{2}\left(\mathrm{CH}_{2}\right)_{2}\left(\mathrm{CH}_{2}\right)_{2} \mathrm{ND}_{2}$ & & & $1.32-1.36(4 \mathrm{H})$ & \\
\hline $\mathrm{OCH}_{2} \mathrm{CH}_{2}\left(\mathrm{CH}_{2}\right)_{2} \mathrm{CH}_{2} \mathrm{CH}_{2} \mathrm{ND}_{2}$ & & & $1.52-1.55(2 \mathrm{H}), 1.62-1.65(2 \mathrm{H})$ & \\
\hline $\mathrm{CH}_{2} \mathrm{ND}_{2}$ & & & 2.97 & \\
\hline $\mathrm{OCH}_{2}\left(\mathrm{CH}_{2}\right)_{5} \mathrm{ND}_{2}$ & & & $3.56,3.83$ & \\
\hline $\mathrm{NDCOCH}_{3}$ & & & $2.02,2.04$ & \\
\hline
\end{tabular}

\footnotetext{
${ }^{\text {a }} \operatorname{GlcNAc}\left(\beta 1-\mathrm{O}\left(\mathrm{CH}_{2}\right)_{6} \mathrm{NH}_{2}\right)$.

b $\operatorname{GlcNAc}(\beta 1-3)$ Gal.

c n.d., not determined.
} 
Table 10

$500 \mathrm{MHz}{ }^{1} \mathrm{H}$ NMR data (TOCSY, ROESY) of 4 at $300 \mathrm{~K}$ (in ppm)

\begin{tabular}{|c|c|c|c|c|c|c|}
\hline \multirow[t]{2}{*}{ Proton } & \multicolumn{6}{|l|}{$\delta_{\mathrm{H}}$} \\
\hline & GlcNAc $\mathrm{I}^{\mathrm{a}}$ & Glc II & Gal III ${ }^{b}$ & GlcNAc IV $^{c}$ & Gal V d & $\mathrm{Gal} \mathrm{VI}^{\mathrm{e}}$ \\
\hline $\mathrm{H}-1$ & 4.53 & 4.56 & 4.44 & 4.71 & 4.53 & 4.48 \\
\hline $\mathrm{H}-2$ & 3.72 & 3.37 & 3.59 & 3.80 & 3.55 & 3.55 \\
\hline $\mathrm{H}-3$ & n.d. ${ }^{\mathrm{f}}$ & 3.67 & 3.72 & 3.73 & 3.67 & 3.67 \\
\hline $\mathrm{H}-4$ & 3.83 & 3.67 & 4.16 & 3.73 & 3.93 & 3.92 \\
\hline H-5 & 3.72 & 3.62 & n.d. & 3.58 & n.d. & n.d. \\
\hline H-6a & 4.29 & 4.00 & n.d. & 3.95 & n.d. & n.d. \\
\hline $\mathrm{H}-6 \mathrm{~b}$ & 3.95 & 3.83 & n.d. & 3.85 & n.d. & n.d. \\
\hline $\mathrm{O}\left(\mathrm{CH}_{2}\right)_{2}\left(\mathrm{CH}_{2}\right)_{2}\left(\mathrm{CH}_{2}\right)_{2} \mathrm{ND}_{2}$ & & & $1.36-1.38(4 \mathrm{H})$ & & & \\
\hline $\mathrm{OCH}_{2} \mathrm{CH}_{2}\left(\mathrm{CH}_{2}\right)_{2} \mathrm{CH}_{2} \mathrm{CH}_{2} \mathrm{ND}_{2}$ & & & $1.54-1.57(2 \mathrm{H}), 1.64-1.67(2 \mathrm{H})$ & & & \\
\hline $\mathrm{CH}_{2} \mathrm{ND}_{2}$ & & & 2.99 & & & \\
\hline $\mathrm{OCH}_{2}\left(\mathrm{CH}_{2}\right)_{5} \mathrm{ND}_{2}$ & & & $3.68,3.92$ & & & \\
\hline $\mathrm{NDCOCH}_{3}$ & & & $2.03(2)$ & & & \\
\hline
\end{tabular}

a $\operatorname{GlcNAc}\left(\beta 1-\mathrm{O}\left(\mathrm{CH}_{2}\right)_{6} \mathrm{NH}_{2}\right)$.

b $\mathrm{Gal}(\beta 1-4) \mathrm{Glc}$.

c GlcNAc( $(\beta 1-3)$ Gal.

${ }^{d} \mathrm{Gal}(\beta 1-4) \mathrm{GlcNAc}\left(\beta 1-\mathrm{O}\left(\mathrm{CH}_{2}\right)_{6} \mathrm{NH}_{2}\right)$.

e $\operatorname{Gal}(\beta 1-4) \mathrm{GlcNAc}(\beta 1-3) \mathrm{Gal}$.

${ }^{\mathrm{f}}$ n.d., not determined.

filtered, and concentrated. Low-pressure column chromatography $(5: 1 \rightarrow 1: 1$ toluene-EtOAc) of the residue gave 18, isolated as a colourless glass $(0.19 \mathrm{~g}, 83 \%) ; R_{f}$ $0.53\left(1: 1\right.$ toluene-EtOAc); $[\alpha]_{\mathrm{D}}^{20}+9^{\circ}\left(c 1, \mathrm{CHCl}_{3}\right) ;{ }^{1} \mathrm{H}$ NMR (500 MHz, $\mathrm{CDCl}_{3} ;$ 2D TOCSY, ROESY): $\delta 1.05$ [s, $\left.9 \mathrm{H}, \mathrm{SiC}\left(\mathrm{CH}_{3}\right)_{3}\right], 1.19-1.22\left(\mathrm{~m}, 4 \mathrm{H}, 2 \mathrm{CH}_{2}\right), 1.37-$ $1.40\left(\mathrm{~m}, 2 \mathrm{H}, \mathrm{CH}_{2}\right), 1.46-1.48\left(\mathrm{~m}, 2 \mathrm{H}, \mathrm{CH}_{2}\right), 1.96(\mathrm{~s}, 3$ $\left.\mathrm{H}, \mathrm{COCH}_{3}\right), 3.07\left(\mathrm{t}, 2 \mathrm{H}, \mathrm{CH}_{2} \mathrm{~N}_{3}\right), 3.33\left(\mathrm{dd}, 1 \mathrm{H}, J_{1,2}\right.$ 7.9, $\left.J_{2,3} 9.8 \mathrm{~Hz}, \mathrm{H}-2^{\mathrm{I}}\right), 3.37(\mathrm{~m}, 1 \mathrm{H}, \mathrm{OC} H \mathrm{H}), 3.78(\mathrm{dd}, 1$ $\left.\mathrm{H}, J_{3,4} 3.5 \mathrm{~Hz}, \mathrm{H}_{-}{ }^{\mathrm{I}}\right), 3.82(\mathrm{~m}, 1 \mathrm{H}, \mathrm{OCH} H), 4.04(\mathrm{dd}, 1$ $\left.\mathrm{H}, J_{1,2} 8.3, J_{2,3} 11.0 \mathrm{~Hz}, \mathrm{H}-2^{\mathrm{II}}\right), 4.17$ and $4.53(2 \mathrm{~d}$, each 1 $\left.\mathrm{H}, \mathrm{OC}_{2} \mathrm{C}_{6} \mathrm{H}_{5}\right), 4.20\left(\mathrm{~d}, 1 \mathrm{H}, \mathrm{H}^{\mathrm{I}}{ }^{\mathrm{I}}\right), 4.37\left(\mathrm{t}, 1 \mathrm{H}, \mathrm{H}-3^{\mathrm{II}}\right)$, 4.38 and $4.43\left(2 \mathrm{~d}\right.$, each $\left.1 \mathrm{H}, \mathrm{OCH}_{2} \mathrm{C}_{6} \mathrm{H}_{5}\right), 5.33(\mathrm{~d}, 1 \mathrm{H}$, $\left.J_{4,5}<1 \mathrm{~Hz}, \mathrm{H}-4^{\mathrm{I}}\right), 5.41\left(\mathrm{~d}, 1 \mathrm{H}, \mathrm{H}-1^{\mathrm{II}}\right) ;{ }^{13} \mathrm{C}$ NMR $(75.5$ $\left.\mathrm{MHz}, \mathrm{CDCl}_{3}\right): \delta 19.0\left[\mathrm{SiC}\left(\mathrm{CH}_{3}\right)_{3}\right], 20.5\left(\mathrm{COCH}_{3}\right), 25.3$, 26.2, 28.4, and $29.2\left(4 \mathrm{CH}_{2}\right), 26.6\left[\mathrm{SiC}\left(\mathrm{CH}_{3}\right)_{3}\right], 51.0$ $\left(\mathrm{CH}_{2} \mathrm{~N}_{3}\right), 56.6\left(\mathrm{C}-2^{\mathrm{II}}\right), 64.5,68.9,69.8,73.3$, and $73.9(\mathrm{C}-$ $\left.6^{\mathrm{I}}, \mathrm{C}-6^{\mathrm{II}}, 2 \mathrm{OCH}_{2} \mathrm{C}_{6} \mathrm{H}_{5}, \mathrm{OCH}_{2}\right), 70.1,71.0,72.5,73.6$, 74.8, 76.4, and $78.6\left(\mathrm{C}-2^{\mathrm{I}}, \mathrm{C}-3^{\mathrm{I}}, \mathrm{C}-4^{\mathrm{I}}, \mathrm{C}-5^{\mathrm{I}}, \mathrm{C}-3^{\mathrm{II}}, \mathrm{C}-4^{\mathrm{II}}\right.$, $\left.\mathrm{C}-5^{\mathrm{II}}\right), 98.0$ and $103.4\left(\mathrm{C}-1^{\mathrm{I}}, \mathrm{C}-1^{\mathrm{II}}\right), 170.0\left(\mathrm{COCH}_{3}\right)$; HRMS of $\mathrm{C}_{58} \mathrm{H}_{68} \mathrm{~N}_{4} \mathrm{O}_{13} \mathrm{Si}(\mathrm{M}, 1056.455)$ : $\left[\mathrm{M}+\mathrm{NH}_{4}\right]^{+}$ found 1074.496, calcd 1074.489.

3.8. 6-Azidohexyl (6-O-tert-butyldiphenylsilyl-2-deoxy3,4-di- $O$ - $p$-methylbenzoyl-2-phthalimido- $\beta$-Dglucopyranosyl)-(1 $\rightarrow 3)-4-O$-acetyl-2,6-di- $O$-benzyl- $\beta$-Dgalactopyranoside (19)

To a soln of $18(0.19 \mathrm{~g}, 0.19 \mathrm{mmol})$ in dry Py $(4 \mathrm{~mL})$ was added dropwise, at $0{ }^{\circ} \mathrm{C}$, a soln of $p$-methylbenzoyl chloride $(60 \mu \mathrm{L}, 0.48 \mathrm{mmol})$ in dry $\mathrm{CH}_{2} \mathrm{Cl}_{2}(1 \mathrm{~mL})$. The mixture was stirred for $4 \mathrm{~h}$ at $\mathrm{rt}$, then poured into ice water, extracted with $\mathrm{CH}_{2} \mathrm{Cl}_{2}$, washed with aq satd $\mathrm{NaHCO}_{3}$, dried $\left(\mathrm{MgSO}_{4}\right)$, filtered, and concentrated. Column chromatography (5:1 toluene-EtOAc) of the residue gave 19, isolated as a colourless syrup $(0.21 \mathrm{~g}$,

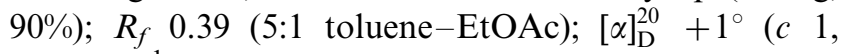
$\left.\mathrm{CHCl}_{3}\right) ;{ }^{1} \mathrm{H}$ NMR $\left(500 \mathrm{MHz}, \mathrm{CDCl}_{3} ; 2 \mathrm{D}\right.$ TOCSY, ROESY): $\delta 1.06\left[\mathrm{~s}, 9 \mathrm{H}, \mathrm{SiC}\left(\mathrm{CH}_{3}\right)_{3}\right], 1.23-1.26(\mathrm{~m}, 4 \mathrm{H}$, $\left.2 \mathrm{CH}_{2}\right), 1.38-1.41\left(\mathrm{~m}, 2 \mathrm{H}, \mathrm{CH}_{2}\right), 1.52-1.54(\mathrm{~m}, 2 \mathrm{H}$, $\left.\mathrm{CH}_{2}\right), 2.04\left(\mathrm{~s}, 3 \mathrm{H}, \mathrm{COCH}_{3}\right), 2.18$ and $2.28(2 \mathrm{~s}$, each 3 $\left.\mathrm{H}, 2 \mathrm{COC}_{6} \mathrm{H}_{4} \mathrm{CH}_{3}\right), 3.07\left(\mathrm{t}, 2 \mathrm{H}, \mathrm{CH}_{2} \mathrm{~N}_{3}\right), 3.64(\mathrm{t}, 1 \mathrm{H}$, H-6a $\left.{ }^{\mathrm{I}}\right), 3.83\left(\mathrm{~d}, 1 \mathrm{H}, J_{6 \mathrm{a}, 6 \mathrm{~b}} 9.4 \mathrm{~Hz}, \mathrm{H}-6 \mathrm{~b}^{\mathrm{II}}\right), 3.89(\mathrm{~m}, 1 \mathrm{H}$, OCH $H), 4.00\left(\mathrm{dd}, 1 \mathrm{H}, J_{2,3} 9.6, J_{3,4} 3.5 \mathrm{~Hz}, \mathrm{H}-3^{\mathrm{I}}\right), 4.24$ and $4.65\left(2 \mathrm{~d}\right.$, each $\left.1 \mathrm{H}, \mathrm{OCH}_{2} \mathrm{C}_{6} \mathrm{H}_{5}\right), 4.30\left(\mathrm{~d}, 1 \mathrm{H}, J_{1,2}\right.$ $\left.7.7 \mathrm{~Hz}, \mathrm{H}-1^{\mathrm{I}}\right), 4.43$ and $4.49\left(2 \mathrm{~d}\right.$, each $\left.1 \mathrm{H}, \mathrm{OCH}_{2} \mathrm{C}_{6} \mathrm{H}_{5}\right)$, $4.53\left(\mathrm{dd}, 1 \mathrm{H}, J_{1,2} 8.3, J_{2,3} 10.7 \mathrm{~Hz}, \mathrm{H}-2^{\mathrm{II}}\right), 5.56(\mathrm{t}, 1 \mathrm{H}$, $\left.\mathrm{H}-4^{\mathrm{II}}\right), 5.59\left(\mathrm{~d}, 1 \mathrm{H}, J_{4,5}<1 \mathrm{~Hz}, \mathrm{H}-4^{\mathrm{I}}\right), 5.84(\mathrm{~d}, 1 \mathrm{H}, \mathrm{H}-$ $\left.1^{\mathrm{II}}\right), 6.31\left(\mathrm{dd}, 1 \mathrm{H}, J_{3,4} 9.2 \mathrm{~Hz}, \mathrm{H}-3^{\mathrm{II}}\right), 6.97$ and $7.08(2 \mathrm{~d}$, each $2 \mathrm{H}$, Phth); ${ }^{13} \mathrm{C}$ NMR (75.5 MHz, $\left.\mathrm{CDCl}_{3}\right): \delta 19.1$ $\left[\begin{array}{llllll} & \mathrm{SiC}\left(\mathrm{CH}_{3}\right)_{3}\end{array}\right], \quad 20.5 \quad\left(\mathrm{COCH}_{3}\right), \quad 21.4$ and $21.5 \quad$ (2 $\left.\mathrm{COC}_{6} \mathrm{H}_{4} \mathrm{CH}_{3}\right), 25.5,26.3,28.5$, and $29.4\left(4 \mathrm{CH}_{2}\right), 26.6$ [SiC $\left.\left(\mathrm{CH}_{3}\right)_{3}\right], 51.1\left(\mathrm{CH}_{2} \mathrm{~N}_{3}\right), 55.5\left(\mathrm{C}-2^{\mathrm{II}}\right), 63.0,69.1,69.8$, 73.4, and 73.9 (C-6 $\left.{ }^{\mathrm{I}}, \mathrm{C}-6^{\mathrm{II}}, 2 \mathrm{OCH}_{2} \mathrm{C}_{6} \mathrm{H}_{5}, \mathrm{OCH}_{2}\right), 69.9$, $70.3,70.8,72.8,75.1,76.4$, and $78.7\left(\mathrm{C}-2^{\mathrm{I}}, \mathrm{C}-3^{\mathrm{I}}, \mathrm{C}-4^{\mathrm{I}}, \mathrm{C}-\right.$ $\left.5^{\mathrm{I}}, \mathrm{C}-3^{\mathrm{II}}, \mathrm{C}-4^{\mathrm{II}}, \mathrm{C}-5^{\mathrm{II}}\right), 98.2$ and $103.6\left(\mathrm{C}-1^{\mathrm{I}}, \mathrm{C}-1^{\mathrm{II}}\right), 164.9$ and $165.5\left(2 \mathrm{COC}_{6} \mathrm{H}_{4} \mathrm{CH}_{3}\right), 169.7\left(\mathrm{COCH}_{3}\right)$; HRMS of $\mathrm{C}_{74} \mathrm{H}_{80} \mathrm{~N}_{4} \mathrm{O}_{17} \mathrm{Si} \quad(\mathrm{M}, 1292.538):\left[\mathrm{M}+\mathrm{NH}_{4}\right]^{+}$found 1310.584, calcd 1310.573 . 
3.9. 6-Azidohexyl (2-deoxy-3,4-di- $O$-p -methylbenzoyl-2phthalimido- $\beta$-D-glucopyranosyl)-(1 $\rightarrow 3)-4-O$-acetyl-2,6di- $O$-benzyl- $\beta$-D-galactopyranoside (20)

To a soln of acetyl chloride $(0.80 \mathrm{~mL}, 9.70 \mathrm{mmol})$ in dry $\mathrm{MeOH}(15 \mathrm{~mL})$ was added, at $0{ }^{\circ} \mathrm{C}$, a soln of $\mathbf{1 9}(0.88 \mathrm{~g}$, $0.71 \mathrm{mmol})$ in dry toluene $(3 \mathrm{~mL})$. The mixture was stirred for $4 \mathrm{~h}$ at $0{ }^{\circ} \mathrm{C}$, then co-concentrated with toluene. Column chromatography (4:1 toluene-EtOAc) of the residue gave $\mathbf{2 0}$, isolated as a colourless syrup $(0.61 \mathrm{~g}, 81 \%) ; R_{f} 0.44$ (2:1 toluene-EtOAc); $[\alpha]_{\mathrm{D}}^{20}+9^{\circ}(c$ $\left.1, \mathrm{CHCl}_{3}\right) ;{ }^{1} \mathrm{H}$ NMR $\left(500 \mathrm{MHz}, \mathrm{CDCl}_{3} ; 2 \mathrm{D}\right.$ TOCSY, ROESY): $\delta 1.12-1.17$ (m, $\left.4 \mathrm{H}, 2 \mathrm{CH}_{2}\right), 1.32-1.35$ (m, 2 $\left.\mathrm{H}, \mathrm{CH}_{2}\right), 1.40-1.44\left(\mathrm{~m}, 2 \mathrm{H}, \mathrm{CH}_{2}\right), 2.18(\mathrm{~s}, 3 \mathrm{H}$, $\left.\mathrm{COCH}_{3}\right), 2.26$ and $2.33\left(2 \mathrm{~s}\right.$, each $\left.3 \mathrm{H}, 2 \mathrm{COC}_{6} \mathrm{H}_{4} \mathrm{CH}_{3}\right)$, $3.05\left(\mathrm{CH}_{2} \mathrm{~N}_{3}\right), 3.34(\mathrm{~m}, 1 \mathrm{H}, \mathrm{OCH}), 3.49\left(\mathrm{dd}, 1 \mathrm{H}, J_{1,2}\right.$ 7.7, $\left.J_{2,3} 9.4 \mathrm{~Hz}, \mathrm{H}-2^{\mathrm{I}}\right), 3.51\left(\mathrm{~d}, 2 \mathrm{H}, J_{6 \mathrm{a}, 6 \mathrm{~b}} 5.8 \mathrm{~Hz}, \mathrm{H}-6 \mathrm{a}^{\mathrm{I}}\right.$, $\left.\mathrm{H}-6 \mathrm{~b}^{\mathrm{I}}\right), 3.82(\mathrm{~m}, 1 \mathrm{H}, \mathrm{OCH} H), 3.90\left(\mathrm{~m}, 1 \mathrm{H}, \mathrm{H}-5^{\mathrm{II}}\right), 4.21$ and $4.45\left(2 \mathrm{~d}\right.$, each $\left.1 \mathrm{H}, \mathrm{OCH}_{2} \mathrm{C}_{6} \mathrm{H}_{5}\right), 4.27(\mathrm{~d}, 1 \mathrm{H}, \mathrm{H}-$ $\left.1^{\mathrm{I}}\right), 4.48\left(\mathrm{~s}, 2 \mathrm{H}, \mathrm{OCH}_{2} \mathrm{C}_{6} \mathrm{H}_{5}\right), 4.51\left(\mathrm{dd}, 1 \mathrm{H}, J_{1,2} 8.3, J_{2,3}\right.$ $\left.10.7 \mathrm{~Hz}, \mathrm{H}-2^{\mathrm{II}}\right), 5.43\left(\mathrm{t}, 1 \mathrm{H}, \mathrm{H}-4^{\mathrm{II}}\right), 5.60\left(\mathrm{~d}, 1 \mathrm{H}, J_{3,4} 3.5\right.$, $\left.J_{4,5}<1 \mathrm{~Hz}, \mathrm{H}-4^{\mathrm{I}}\right), 5.72\left(\mathrm{~d}, 1 \mathrm{H}, \mathrm{H}-1^{\mathrm{II}}\right), 6.13\left(\mathrm{dd}, 1 \mathrm{H}, J_{3,4}\right.$ $\left.9.2 \mathrm{~Hz}, \mathrm{H}-3{ }^{\mathrm{II}}\right) ;{ }^{13} \mathrm{C}$ NMR $\left(75.5 \mathrm{MHz}, \mathrm{CDCl}_{3}\right): \delta 21.0$ $\left(\mathrm{COCH}_{3}\right), 21.4$ and $21.5\left(2 \mathrm{COC}_{6} \mathrm{H}_{4} \mathrm{CH}_{3}\right), 25.3,26.2$, 28.4, and $29.2\left(4 \mathrm{CH}_{2}\right), 51.1\left(\mathrm{CH}_{2} \mathrm{~N}_{3}\right), 55.0\left(\mathrm{C}-2^{\mathrm{II}}\right), 61.5$, 70.0, 73.6, 68.8, and $73.9\left(\mathrm{C}-6^{\mathrm{I}}, \mathrm{C}-6^{\mathrm{II}}, 2 \mathrm{OCH}_{2} \mathrm{C}_{6} \mathrm{H}_{5}\right.$, $\left.\mathrm{OCH}_{2}\right), 69.3,70.1,70.8,72.4,75.2,77.5$, and $81.5\left(\mathrm{C}-2^{\mathrm{I}}\right.$, $\left.\mathrm{C}-3^{\mathrm{I}}, \mathrm{C}-4^{\mathrm{I}}, \mathrm{C}-5^{\mathrm{I}}, \mathrm{C}-3^{\mathrm{II}}, \mathrm{C}-4^{\mathrm{II}}, \mathrm{C}-5^{\mathrm{II}}\right), 99.4$ and 103.5 (C$\left.1^{\mathrm{I}}, \mathrm{C}-1^{\mathrm{II}}\right), 165.2$ and $165.4\left(2 \mathrm{COC}_{6} \mathrm{H}_{4} \mathrm{CH}_{3}\right), 171.4$ $\left(\mathrm{COCH}_{3}\right)$; HRMS of $\mathrm{C}_{58} \mathrm{H}_{62} \mathrm{~N}_{4} \mathrm{O}_{15}(\mathrm{M}, 1054.421)$ : $\left[\mathrm{M}+\mathrm{NH}_{4}\right]^{+}$found 1072.461, calcd 1072.455.

\subsection{6-Azidohexyl $(2,3,4,6$-tetra- $O$-acetyl- $\beta$-D- glucopyranosyl)-(1 $\rightarrow 6)-(2-$ deoxy-3,4-di- $O-p$ - methylbenzoyl-2-phthalimido- $\beta$-D-glucopyranosyl)-( $1 \rightarrow$ 3)-4- $O$-acetyl-2,6-di- $O$-benzyl- $\beta$-D-galactopyranoside}

(21)

(a) A soln of 2,3,4,6-tetra- $O$-acetyl- $\alpha$-D-glucopyranosyl trichloroacetimidate $(9)^{16}(0.40 \mathrm{~g}, 0.82 \mathrm{mmol})$ and 20 $(0.48 \mathrm{~g}, 0.45 \mathrm{mmol})$ in dry $\mathrm{CH}_{2} \mathrm{Cl}_{2}(20 \mathrm{~mL})$, containing 4 $\AA$ molecular sieves $(0.5 \mathrm{~g})$, was stirred under $\mathrm{Ar}$ for 0.5 h. After cooling to $-40{ }^{\circ} \mathrm{C}$, TMSOTf $(14 \mu \mathrm{L}, 0.077$ mmol) was added, and the mixture was stirred for $2.5 \mathrm{~h}$, during which period the temperature was allowed to reach $\mathrm{rt}$, then neutralized with $\mathrm{Et}_{3} \mathrm{~N}$, filtered, and concentrated. Column chromatography (4:1 tolueneEtOAc) of the residue gave 21, isolated as white foam $(0.35 \mathrm{~g}, 56 \%)$.

(b) A soln of $13(0.13 \mathrm{~g}, 0.13 \mathrm{mmol})$ and 6-azidohexyl 4- $O$-acetyl-2,6-di- $O$-benzyl- $\beta$-D-galactopyranoside (14) $)^{14}(56 \mathrm{mg}, 0.11 \mathrm{mmol})$ in dry $\mathrm{CH}_{2} \mathrm{Cl}_{2}(3 \mathrm{~mL})$, containing $4 \AA$ molecular sieves $(0.1 \mathrm{~g})$, was stirred under Ar for $0.5 \mathrm{~h}$. After cooling to $-70{ }^{\circ} \mathrm{C}$, TMSOTf $(2.2 \mu \mathrm{L}, 0.013 \mathrm{mmol})$ was added, and the mixture was stirred for $2.5 \mathrm{~h}$, during which period the temperature was allowed to reach $\mathrm{rt}$, then neutralized with $\mathrm{Et}_{3} \mathrm{~N}$, filtered, and concentrated. Column chromatography (4:1 toluene-EtOAc) of the residue gave 21, isolated as a white foam $(0.13 \mathrm{~g}, 73 \%) ; R_{f} 0.54$ (2:1 tolueneEtOAc); $[\alpha]_{\mathrm{D}}^{20}+8^{\circ}\left(c 1, \mathrm{CHCl}_{3}\right) ;{ }^{1} \mathrm{H}$ NMR $(300 \mathrm{MHz}$, $\left.\mathrm{CDCl}_{3}\right): \delta 1.23-1.25\left(\mathrm{~m}, 4 \mathrm{H}, 2 \mathrm{CH}_{2}\right), 1.39-1.43(\mathrm{~m}, 2$ $\left.\mathrm{H}, \mathrm{CH}_{2}\right), 1.47-1.52\left(\mathrm{~m}, 2 \mathrm{H}, \mathrm{CH}_{2}\right), 1.95,1.98,2.02,2.05$, and $2.09\left(5 \mathrm{~s}\right.$, each $\left.3 \mathrm{H}, 5 \mathrm{COCH}_{3}\right), 2.27$ and $2.34(2 \mathrm{~s}$, each $\left.3 \mathrm{H}, 2 \mathrm{COC}_{6} \mathrm{H}_{4} \mathrm{CH}_{3}\right), 3.11\left(\mathrm{t}, 2 \mathrm{H}, \mathrm{CH}_{2} \mathrm{~N}_{3}\right), 3.38$ $\left(\mathrm{dd}, 1 \mathrm{H}, J_{1,2} 7.8, J_{2,3} 9.5 \mathrm{~Hz}, \mathrm{H}-2^{\mathrm{I}}\right), 3.42(\mathrm{~m}, 1 \mathrm{H}$, $\mathrm{OC} H \mathrm{H}), 4.07$ (dd, $1 \mathrm{H}, J_{5,6 \mathrm{~b}} 4.4, J_{6 \mathrm{a}, 6 \mathrm{~b}} 12.3 \mathrm{~Hz}, \mathrm{H}-6 \mathrm{~b}^{\mathrm{III}}$ ), 4.10 and $4.55\left(2 \mathrm{~d}\right.$, each $\left.1 \mathrm{H}, \mathrm{OCH}_{2} \mathrm{C}_{6} \mathrm{H}_{5}\right), 4.36(\mathrm{~d}, 1 \mathrm{H}$, $\left.\mathrm{H}-1^{\mathrm{I}}\right), 4.44\left(\mathrm{dd}, 1 \mathrm{H}, J_{1,2} 8.2, J_{2,3} 10.8 \mathrm{~Hz}, \mathrm{H}-2^{\mathrm{II}}\right), 4.47$ and $4.56\left(2 \mathrm{~d}\right.$, each $\left.1 \mathrm{H}, \mathrm{OCH}_{2} \mathrm{C}_{6} \mathrm{H}_{5}\right), 4.70\left(\mathrm{~d}, 1 \mathrm{H}, J_{1,2}\right.$ $\left.8.0 \mathrm{~Hz}, \mathrm{H}-1^{\mathrm{III}}\right), 4.95\left(\mathrm{dd}, 1 \mathrm{H}, J_{2,3} 9.6 \mathrm{~Hz}, \mathrm{H}-2^{\mathrm{III}}\right), 5.01(\mathrm{t}$, $\left.1 \mathrm{H}, \mathrm{H}-4^{\mathrm{III}}\right), 5.21\left(\mathrm{t}, 1 \mathrm{H}, \mathrm{H}-3^{\mathrm{III}}\right), 5.36\left(\mathrm{t}, 1 \mathrm{H}, \mathrm{H}-4^{\mathrm{II}}\right)$, $5.55\left(\mathrm{~d}, 1 \mathrm{H}, J_{3,4} 3.5, J_{4,5}<1 \mathrm{~Hz}, \mathrm{H}-4^{\mathrm{I}}\right), 5.68(\mathrm{~d}, 1 \mathrm{H}, \mathrm{H}-$ $\left.1^{\mathrm{II}}\right), 6.24\left(\mathrm{dd}, 1 \mathrm{H}, J_{3,4} 9.1 \mathrm{~Hz}, \mathrm{H}-3{ }^{\mathrm{II}}\right) ;{ }^{13} \mathrm{C}$ NMR $(75.5$ $\left.\mathrm{MHz}, \mathrm{CDCl}_{3}\right): \delta 20.4-20.8\left(\mathrm{COCH}_{3}\right), 21.4$ and $21.5(2$ $\left.\mathrm{COC}_{6} \mathrm{H}_{4} \mathrm{CH}_{3}\right), 25.5,26.3,28.5$, and $29.3\left(4 \mathrm{CH}_{2}\right), 51.2$ $\left(\mathrm{CH}_{2} \mathrm{~N}_{3}\right), 55.3\left(\mathrm{C}-2^{\mathrm{II}}\right), 61.5,68.0(2 \mathrm{C}), 69.9,73.4$, and 73.9 (C-6 $\left.^{\mathrm{I}}, \mathrm{C}-6^{\mathrm{II}}, \mathrm{C}-6^{\mathrm{III}}, 2 \mathrm{OCH}_{2} \mathrm{C}_{6} \mathrm{H}_{5}, \mathrm{OCH}_{2}\right), 67.9$, $69.7,69.9,70.3,71.5,71.6,71.8,72.7,74.5,77.2$, and $78.5\left(\mathrm{C}-2^{\mathrm{I}}, \mathrm{C}-3^{\mathrm{I}}, \mathrm{C}-4^{\mathrm{I}}, \mathrm{C}-5^{\mathrm{I}}, \mathrm{C}-3^{\mathrm{II}}, \mathrm{C}-4^{\mathrm{II}}, \mathrm{C}-5^{\mathrm{II}}, \mathrm{C}-2^{\mathrm{III}}, \mathrm{C}-\right.$ $\left.3^{\mathrm{III}}, \mathrm{C}-4^{\mathrm{III}}, \mathrm{C}-5^{\mathrm{III}}\right), 98.4,100.9$, and $103.6\left(\mathrm{C}-1^{\mathrm{I}}, \mathrm{C}-1^{\mathrm{II}}, \mathrm{C}-\right.$ $\left.1^{\mathrm{III}}\right), 165.1$ and $165.4\left(2 \mathrm{COC}_{6} \mathrm{H}_{4} \mathrm{CH}_{3}\right), 169.1-170.5$ $\left(\mathrm{COCH}_{3}\right)$; HRMS of $\mathrm{C}_{72} \mathrm{H}_{80} \mathrm{~N}_{4} \mathrm{O}_{24}(\mathrm{M}, 1384.516)$ : [M+ $\mathrm{Na}]^{+}$found 1407.495, calcd 1407.506.

\subsection{6-Azidohexyl $(2,3,4,6$-tetra- $O$-acetyl- $\beta$-D- glucopyranosyl)-(1 $\rightarrow$ 6)-(2-acetamido-3,4-di- $O$-acetyl-2- deoxy- $\beta$-D-glucopyranosyl)-(1 $\rightarrow 3)-4-O$-acetyl-2,6-di- $O$ - benzyl- $\beta$-D-galactopyranoside (22)}

To a soln of $21(90 \mathrm{mg}, 64.9 \mu \mathrm{mol})$ in $\mathrm{MeOH}(8 \mathrm{~mL})$ and $\mathrm{CH}_{2} \mathrm{Cl}_{2}(2 \mathrm{~mL})$ was added $\mathrm{NaOMe}(\mathrm{pH} 10)$, and the mixture was stirred for $4 \mathrm{~h}$, then neutralized with Dowex $50 \times 8\left(\mathrm{H}^{+}\right)$, filtered, and concentrated. To a soln of the residue in $1-\mathrm{BuOH}(30 \mathrm{~mL})$ was added 1,2-diaminoethane $(6 \mathrm{~mL})$, and the mixture was stirred overnight at $80{ }^{\circ} \mathrm{C}$, then co-concentrated with toluene, $\mathrm{EtOH}$, and $\mathrm{CH}_{2} \mathrm{Cl}_{2}$. A soln of the residue in $\mathrm{Py}(30 \mathrm{~mL})$ and $\mathrm{Ac}_{2} \mathrm{O}$ $(30 \mathrm{~mL})$ was stirred overnight, then co-concentrated with toluene, EtOH and $\mathrm{CH}_{2} \mathrm{Cl}_{2}$. Column chromatography (1:2 toluene-EtOAc) of the residue gave 22, isolated as a colourless syrup (60 mg, 80\%); $R_{f} 0.27(1: 2$ toluene-EtOAc); $[\alpha]_{\mathrm{D}}^{20}-8^{\circ}\left(c 1, \mathrm{CHCl}_{3}\right) ;{ }^{1} \mathrm{H}$ NMR $(500$ $\mathrm{MHz}, \mathrm{CDCl}_{3}$; 2D TOCSY, ROESY): $\delta 1.32-1.39$ (m, 4 $\left.\mathrm{H}, 2 \mathrm{CH}_{2}\right), 1.47-1.54\left(\mathrm{~m}, 2 \mathrm{H}, \mathrm{CH}_{2}\right), 1.59-1.63(\mathrm{~m}, 2 \mathrm{H}$, $\left.\mathrm{CH}_{2}\right), 1.59,1.95,1.96,1.99,2.06$ and $2.07(6 \mathrm{~s}$, $\left.3,3,3,6,3,6 \mathrm{H}, 7 \mathrm{COCH}_{3}, \mathrm{NHCOCH}_{3}\right), 3.18(\mathrm{t}, 2 \mathrm{H}$, $\left.\mathrm{CH}_{2} \mathrm{~N}_{3}\right), 3.63\left(\mathrm{~m}, 1 \mathrm{H}, \mathrm{H}-5^{\mathrm{III}}\right), 3.71\left(\mathrm{dd}, 1 \mathrm{H}, J_{5,6 \mathrm{~b}} 6.8\right.$, $\left.J_{6 \mathrm{a}, 6 \mathrm{~b}} 12.3 \mathrm{~Hz}, \mathrm{H}-6 \mathrm{~b}^{\mathrm{II}}\right), 3.80\left(\mathrm{dd}, 1 \mathrm{H}, J_{5,6 \mathrm{a}} 1.5 \mathrm{~Hz}, \mathrm{H}-\right.$ $\left.6 \mathrm{a}^{\mathrm{II}}\right), 4.19\left(\mathrm{dd}, 1 \mathrm{H}, J_{5,6 \mathrm{~b}} 4.2, J_{6 \mathrm{a}, 6 \mathrm{~b}} 12.5 \mathrm{~Hz}, \mathrm{H}-6 \mathrm{~b}^{\mathrm{III}}\right)$, $4.43\left(\mathrm{~d}, 1 \mathrm{H}, J_{1,2} 8.1 \mathrm{~Hz}, \mathrm{H}-1^{\mathrm{III}}\right), 4.47$ and $4.56(2 \mathrm{~d}$, each $\left.1 \mathrm{H}, \mathrm{OCH}_{2} \mathrm{C}_{6} \mathrm{H}_{5}\right), 4.55$ and $5.04(2 \mathrm{~d}$, each $1 \mathrm{H}$, 
$\left.\mathrm{OCH}_{2} \mathrm{C}_{6} \mathrm{H}_{5}\right), 4.65\left(\mathrm{~d}, 1 \mathrm{H}, J_{1,2} 8.1 \mathrm{~Hz}, \mathrm{H}-1^{\mathrm{I}}\right), 4.73(\mathrm{~d}, 1$ $\left.\mathrm{H}, J_{1,2} 8.3 \mathrm{~Hz}, \mathrm{H}-1^{\mathrm{II}}\right), 4.83\left(\mathrm{t}, 1 \mathrm{H}, \mathrm{H}-4^{\mathrm{II}}\right), 4.89(\mathrm{t}, 1 \mathrm{H}$, $\left.\mathrm{H}-2^{\mathrm{II}}\right), 4.99\left(\mathrm{t}, 1 \mathrm{H}, \mathrm{H}-2^{\mathrm{III}}\right), 5.09\left(\mathrm{t}, 1 \mathrm{H}, \mathrm{H}-4^{\mathrm{III}}\right), 5.25(\mathrm{t}, 1$ $\left.\mathrm{H}, \mathrm{H}-3^{\mathrm{III}}\right), 5.47\left(\mathrm{~d}, 1 \mathrm{H}, J_{3,4} 3.5, J_{4,5}<1 \mathrm{~Hz}, \mathrm{H}-4^{\mathrm{I}}\right) ;{ }^{13} \mathrm{C}$ NMR $\left(75.5 \mathrm{MHz}, \mathrm{CDCl}_{3}\right): \delta$ 20.4-20.7 $\left(\mathrm{COCH}_{3}\right), 22.8$ $\left(\mathrm{NHCOCH}_{3}\right), 25.5,26.3,28.6$, and $29.4\left(4 \mathrm{CH}_{2}\right), 51.2$ $\left(\mathrm{CH}_{2} \mathrm{~N}_{3}\right), 54.1\left(\mathrm{C}-2^{\mathrm{II}}\right), 61.5,68.2(2 \mathrm{C}), 69.8,73.4$, and $74.4\left(\mathrm{C}-6^{\mathrm{I}}, \mathrm{C}-6^{\mathrm{II}}, \mathrm{C}-6^{\mathrm{III}}, 2 \mathrm{OCH}_{2} \mathrm{C}_{6} \mathrm{H}_{5}, \mathrm{OCH}_{2}\right), 68.0$, 69.0, 69.5, 71.6 (2 C), 72.1, 72.4, 72.6, 73.8, 76.9, and 79.9 (C-2 ${ }^{\mathrm{I}}, \mathrm{C}-3^{\mathrm{I}}, \mathrm{C}-4^{\mathrm{I}}, \mathrm{C}-5^{\mathrm{I}}, \mathrm{C}-3^{\mathrm{II}}, \mathrm{C}-4^{\mathrm{II}}, \mathrm{C}-5^{\mathrm{II}}, \mathrm{C}-2^{\mathrm{III}}, \mathrm{C}-$ $\left.3^{\mathrm{III}}, \mathrm{C}-4^{\mathrm{III}}, \mathrm{C}-5^{\mathrm{III}}\right), 100.6,101.2$, and $103.4\left(\mathrm{C}-1^{\mathrm{I}}, \mathrm{C}-1^{\mathrm{II}}\right.$, C-1 $\left.{ }^{\text {III }}\right), 169.1-170.6\left(\mathrm{COCH}_{3}\right) ; \mathrm{HRMS}$ of $\mathrm{C}_{54} \mathrm{H}_{72} \mathrm{~N}_{4} \mathrm{O}_{23}$ (M, 1144.458): $\left[\mathrm{M}+\mathrm{NH}_{4}\right]^{+}$found 1162.489, calcd 1162.492 .

\subsection{6-Aminohexyl $\beta$-D-glucopyranosyl-( $(1 \rightarrow 6)-2-$ acetamido-2-deoxy- $\beta$-D-glucopyranosyl-( $1 \rightarrow 3)$ - $\beta$-D- galactopyranoside (5)}

To a soln of $22(50 \mathrm{mg}, 43.6 \mu \mathrm{mol})$ in $\mathrm{CH}_{2} \mathrm{Cl}_{2}(1 \mathrm{~mL})$ and $\mathrm{MeOH}(1 \mathrm{~mL})$ was added $\mathrm{NaOMe}(\mathrm{pH} 9)$. The mixture was stirred for $3 \mathrm{~h}$, then neutralized with Dowex $50 \times 8\left(\mathrm{H}^{+}\right)$, filtered, and concentrated, giving crude 23 as a white solid (36 mg). To a soln of 23 in tert $-\mathrm{BuOH}(8$ $\mathrm{mL})$ and water $(8 \mathrm{~mL})$ were added $10 \% \mathrm{Pd}-\mathrm{C}(100 \mathrm{mg})$ and 3 drops of aq $25 \% \mathrm{NH}_{3}$. The mixture was stirred for $3 \mathrm{~h}$ under $\mathrm{H}_{2}$ after which $\mathrm{NH}_{3}$ was removed by bubbling with $\mathrm{N}_{2}$, then $10 \% \mathrm{Pd}-\mathrm{C}(60 \mathrm{mg})$ and 3 drops of $\mathrm{AcOH}$ were added, and the stirring under $\mathrm{H}_{2}$ was continued overnight. The mixture was loaded on a short Dowex $50 \times 8\left(\mathrm{H}^{+}\right)$column, which was first eluted with water to remove contaminations, then with aq $10 \% \mathrm{NH}_{4} \mathrm{OH}$ to give $\mathbf{5}$, isolated as a white solid after lyophilization (19 $\mathrm{mg}, 69 \%) ; R_{f} 0.34$ (2:1:1 AcOH-1-BuOH-water); $[\alpha]_{\mathrm{D}}^{20}$ $-2^{\circ}$ (c 1, water); ${ }^{13} \mathrm{C}$ NMR $\left(75.5 \mathrm{MHz}, \mathrm{D}_{2} \mathrm{O}\right): \delta 22.9$ $\left(\mathrm{NDCOCH}_{3}\right), 25.3,26.0,27.9$, and $29.2\left(4 \mathrm{CH}_{2}\right), 40.3$ $\left(\mathrm{CH}_{2} \mathrm{ND}_{2}\right), 56.4\left(\mathrm{C}-2^{\mathrm{II}}\right), 61.5,61.7,69.4$, and $71.1\left(\mathrm{C}-6^{\mathrm{I}}\right.$, $\left.\mathrm{C}-6^{\mathrm{II}}, \mathrm{C}-6^{\mathrm{III}}, \mathrm{OCH}_{2}\right), 69.0,70.4(2 \mathrm{C}), 70.5,73.8,74.3$, 75.4 (2 C), 76.5, 76.7, and $83.1\left(\mathrm{C}-2^{\mathrm{I}}, \mathrm{C}-3^{\mathrm{I}}, \mathrm{C}-4^{\mathrm{I}}, \mathrm{C}-5^{\mathrm{I}}, \mathrm{C}-\right.$ $\left.3^{\mathrm{II}}, \mathrm{C}-4^{\mathrm{II}}, \mathrm{C}-5^{\mathrm{II}}, \mathrm{C}-2^{\mathrm{III}}, \mathrm{C}-3^{\mathrm{III}}, \mathrm{C}-4^{\mathrm{III}}, \mathrm{C}-5^{\mathrm{III}}\right), 103.3$, 103.5, and $103.7 \quad\left(\mathrm{C}-1^{\mathrm{I}}, \quad \mathrm{C}-1^{\mathrm{II}}, \quad \mathrm{C}-1^{\mathrm{III}}\right), \quad 175.7$ $\left(\mathrm{NDCOCH}_{3}\right)$; HRMS of $\mathrm{C}_{26} \mathrm{H}_{48} \mathrm{~N}_{2} \mathrm{O}_{16}(\mathrm{M}, 644.300)$ : $[\mathrm{M}+\mathrm{H}]^{+}$found 645.306 , calcd 645.308. For ${ }^{1} \mathrm{H}$ NMR data, see Table 1 .

\subsection{6-Aminohexyl $\beta$-D-glucopyranosyl-( $1 \rightarrow 6)-[\beta$-D- galactopyranosyl-( $1 \rightarrow 4)]$-2-acetamido-2-deoxy- $\beta$-D- glucopyranosyl-( $1 \rightarrow 3)$ - $\beta$-D-galactopyranoside (1)}

To a soln of $5(9.9 \mathrm{mg}, 15.35 \mu \mathrm{mol})$ in aq $50 \mathrm{mM}$ sodium cacodylate buffer $\mathrm{pH} 7.5(700 \mu \mathrm{L})$, containing $5 \mathrm{mM}$ $\mathrm{MnCl}_{2}$, bovine serum albumin (BSA) $(0.5 \mathrm{mg}$ ), and $\mathrm{NaN}_{3}(0.02 \%)$, were added alkaline phosphatase (14 U), UDP-galactose (13 mg, $21.3 \mu \mathrm{mol}$ ), and $\beta$-1,4-Galacto- syltransferase ( $3 \mathrm{U})$. The mixture was incubated for $20 \mathrm{~h}$ at $37^{\circ} \mathrm{C}$, then water $(100 \mu \mathrm{L})$ was added. UDP-galactose was removed using a Dowex $1 \times 8\left(\mathrm{Cl}^{-}\right)$column with water as eluent. The eluate was concentrated, and the residue applied to a Bio-Gel P-2 column eluted with aq $0.1 \mathrm{M} \mathrm{NH}_{4} \mathrm{HCO}_{3}$ at a flow rate of $40 \mathrm{~mL} / \mathrm{h}$. The appropriate fractions were freeze-dried to give $\mathbf{1}(7.2 \mathrm{mg}$, $54 \%)$ and 47 (5.2 $\mathrm{mg}, 35 \%) ; R_{f} 0.20(2: 1: 1 \mathrm{AcOH}-1-$ $\mathrm{BuOH}$-water); $[\alpha]_{\mathrm{D}}^{20}-3^{\circ}$ (c 0.5 , water); HRMS of $\mathrm{C}_{32} \mathrm{H}_{58} \mathrm{~N}_{2} \mathrm{O}_{21}(\mathrm{M}, 806.353):[\mathrm{M}+\mathrm{H}]^{+}$found 807.352, calcd 807.361. For ${ }^{1} \mathrm{H}$ NMR data, see Table 2.

3.14. 6-Azidohexyl (3,4,6-tri- $O$-acetyl-2-deoxy-2phthalimido- $\beta$-D-glucopyranosyl)-( $1 \rightarrow 3)-(4-O$-acetyl-2,6di- $O$-benzyl- $\beta$-D-galactopyranosyl)-(1 $\rightarrow 4)$-2,3,6-tri- $O$ benzyl- $\beta$-D-glucopyranoside (25)

A soln of 3,4,6-tri- $O$-acetyl-2-deoxy-2-phthalimido- $\beta$-Dglucopyranosyl trichloroacetimidate $(\mathbf{1 5})^{17}(0.48 \mathrm{~g}, 0.77$ mmol) and 6-azidohexyl (4- $O$-acetyl-2,6-di- $O$-benzyl- $\beta$ D-galactopyranosyl)-( $(\rightarrow 4)-2,3,6$-tri- $O$-benzyl- $\beta$-D-glucopyranoside $(\mathbf{2 4})^{14}(0.62 \mathrm{~g}, 0.64 \mathrm{mmol})$ in dry $\mathrm{CH}_{2} \mathrm{Cl}_{2}$ $(15 \mathrm{~mL})$, containing $4 \AA$ powdered molecular sieves $(0.5$ g), was stirred under Ar for $0.5 \mathrm{~h}$. After cooling to $-70{ }^{\circ} \mathrm{C}$, TMSOTf $(14 \mu \mathrm{L}, 0.077 \mathrm{mmol})$ was added, and the mixture was stirred for $2.5 \mathrm{~h}$, during which period the temperature was allowed to reach rt, then neutralized with $\mathrm{Et}_{3} \mathrm{~N}$, filtered over hyflo, washed with water, dried $\left(\mathrm{MgSO}_{4}\right)$, filtered, and concentrated. Column chromatography (2:1 toluene-EtOAc) of the residue gave 25, isolated as a colourless foam $(0.87 \mathrm{~g}, 97 \%) ; R_{f}$ $0.50\left(2: 1\right.$ toluene-EtOAc); $[\alpha]_{\mathrm{D}}^{20}-2^{\circ}\left(c \quad 1, \mathrm{CHCl}_{3}\right) ;{ }^{1} \mathrm{H}$ NMR (300 MHz, $\left.\mathrm{CDCl}_{3}\right): \delta 1.33-1.37\left(\mathrm{~m}, 4 \mathrm{H}, 2 \mathrm{CH}_{2}\right)$, 1.51-1.54 (m, $\left.2 \mathrm{H}, \mathrm{CH}_{2}\right), 1.58-1.60\left(\mathrm{~m}, 2 \mathrm{H}, \mathrm{CH}_{2}\right), 1.82$, 2.02, and $2.06\left(3 \mathrm{~s}, 3,6,3 \mathrm{H}, 4 \mathrm{COCH}_{3}\right), 3.03(\mathrm{~m}, 1 \mathrm{H}, \mathrm{H}-$ $\left.5^{\mathrm{I}}\right), 3.18\left(\mathrm{t}, 2 \mathrm{H}, \mathrm{CH}_{2} \mathrm{~N}_{3}\right), 3.49\left(\mathrm{dd}, 1 \mathrm{H}, J_{5,6 \mathrm{a}} 4.1, J_{6 \mathrm{a}, 6 \mathrm{~b}}\right.$ $\left.11.0 \mathrm{~Hz}, \mathrm{H}-6 \mathrm{a}^{\mathrm{I}}\right), 3.58\left(\mathrm{dd}, 1 \mathrm{H}, J_{2,3} 9.5, J_{3,4} 3.5 \mathrm{~Hz}, \mathrm{H}-\right.$ $\left.3^{\mathrm{II}}\right), 3.87\left(\mathrm{t}, 1 \mathrm{H}, \mathrm{H}-4^{\mathrm{I}}\right), 4.15\left(\mathrm{~d}, 1 \mathrm{H}, \mathrm{OC} H \mathrm{HC}_{6} \mathrm{H}_{5}\right), 4.41$ $\left(\mathrm{d}, 1 \mathrm{H}, \mathrm{OCH} H \mathrm{C}_{6} \mathrm{H}_{5}\right), 4.48\left(\mathrm{~d}, 1 \mathrm{H}, \mathrm{OCH} H \mathrm{C}_{6} \mathrm{H}_{5}\right), 4.65$ and $4.88\left(2 \mathrm{~d}\right.$, each $\left.1 \mathrm{H}, \mathrm{OCH}_{2} \mathrm{C}_{6} \mathrm{H}_{5}\right), 4.67$ and $4.81(2 \mathrm{~d}$, each $\left.1 \mathrm{H}, \mathrm{OCH}_{2} \mathrm{C}_{6} \mathrm{H}_{5}\right), 5.16\left(\mathrm{t}, 1 \mathrm{H}, \mathrm{H}-4^{\mathrm{III}}\right), 5.38(\mathrm{~d}, 1 \mathrm{H}$, $\left.J_{4,5}<1 \mathrm{~Hz}, \mathrm{H}-4^{\mathrm{II}}\right), 5.53\left(\mathrm{~d}, 1 \mathrm{H}, J_{1,2} 8.3 \mathrm{~Hz}, \mathrm{H}-1^{\mathrm{III}}\right), 5.78$ (dd, $\left.1 \mathrm{H}, J_{2,3} 10.7, J_{3,4} 9.2 \mathrm{~Hz}, \mathrm{H}-3^{\mathrm{III}}\right) ;{ }^{13} \mathrm{C}$ NMR $(75.5$ $\left.\mathrm{MHz}, \mathrm{CDCl}_{3}\right): \delta 20.0-20.3\left(\mathrm{COCH}_{3}\right), 25.3,26.1,28.4$, and $29.2\left(4 \mathrm{CH}_{2}\right), 50.9\left(\mathrm{CH}_{2} \mathrm{~N}_{3}\right), 54.6\left(\mathrm{C}-2^{\mathrm{III}}\right), 61.3,67.4$, $67.9,69.3,72.2,73.1,74.0,74.5$, and $74.7\left(\mathrm{C}-6^{\mathrm{I}}, \mathrm{C}-6^{\mathrm{II}}, \mathrm{C}-\right.$ $\left.6^{\mathrm{III}}, 5 \mathrm{OCH}_{2} \mathrm{C}_{6} \mathrm{H}_{5}, \mathrm{OCH}_{2}\right), 68.6,69.5,70.2,71.4,72.2$, $74.3,75.3,78.4,78.9,81.2$, and $82.2\left(\mathrm{C}-2^{\mathrm{I}}, \mathrm{C}-3^{\mathrm{I}}, \mathrm{C}-4^{\mathrm{I}}, \mathrm{C}-\right.$ $\left.5^{\mathrm{I}}, \mathrm{C}-2^{\mathrm{II}}, \mathrm{C}-3^{\mathrm{II}}, \mathrm{C}-4^{\mathrm{II}}, \mathrm{C}-5^{\mathrm{II}}, \mathrm{C}-3^{\mathrm{III}}, \mathrm{C}-4^{\mathrm{III}}, \mathrm{C}-5^{\mathrm{III}}\right), 97.9$, 101.4, and $103.1\left(\mathrm{C}-1^{\mathrm{I}}, \mathrm{C}-1^{\mathrm{II}}, \mathrm{C}-1^{\mathrm{III}}\right), 169.0,169.3,169.7$, and $170.3\left(4 \mathrm{COCH}_{3}\right)$; HRMS of $\mathrm{C}_{75} \mathrm{H}_{84} \mathrm{~N}_{4} \mathrm{O}_{21}$ (M, 1376.562): $\quad\left[\mathrm{M}+\mathrm{NH}_{4}\right]^{+}$found: 1394.548, calcd 1394.597. 
3.15. 6-Azidohexyl (2-deoxy-2-phthalimido- $\beta$-Dglucopyranosyl)-( $1 \rightarrow 3)$-(4- $O$-acetyl-2,6-di- $O$-benzyl- $\beta$-Dgalactopyranosyl)-( $1 \rightarrow 4)-2,3,6$-tri- $O$-benzyl- $\beta$-Dglucopyranoside (26)

To a soln of $25(0.85 \mathrm{~g}, 0.62 \mathrm{mmol})$ in $\mathrm{CH}_{2} \mathrm{Cl}_{2}(10 \mathrm{~mL})$ and $\mathrm{MeOH}(20 \mathrm{~mL})$ was added $\mathrm{NaOMe}(\mathrm{pH} 8)$. The mixture was stirred for $3 \mathrm{~h}$, then neutralized with Dowex $50 \times 8\left(\mathrm{H}^{+}\right)$, filtered, and concentrated. Column chromatography $(3: 1 \rightarrow 1: 1$ toluene-EtOAc) of the residue gave 26, isolated as a colourless syrup $(0.60 \mathrm{~g}, 78 \%) ; R_{f}$ 0.35 (1:3 toluene-EtOAc); $[\alpha]_{\mathrm{D}}^{20}+3^{\circ}\left(c 1, \mathrm{CHCl}_{3}\right) ;{ }^{1} \mathrm{H}$ NMR $\left(500 \mathrm{MHz}, \mathrm{CDCl}_{3} ; 2 \mathrm{D}\right.$ TOCSY, ROESY): $\delta$ $1.33-1.36\left(\mathrm{~m}, 4 \mathrm{H}, 2 \mathrm{CH}_{2}\right), 1.50-1.53\left(\mathrm{~m}, 2 \mathrm{H}, \mathrm{CH}_{2}\right)$, 1.57-1.60 (m, $\left.2 \mathrm{H}, \mathrm{CH}_{2}\right), 2.07\left(\mathrm{~s}, 3 \mathrm{H}, \mathrm{COCH}_{3}\right), 2.98(\mathrm{~m}$, $\left.1 \mathrm{H}, \mathrm{H}-5^{\mathrm{I}}\right), 3.17\left(\mathrm{t}, 2 \mathrm{H}, \mathrm{CH}_{2} \mathrm{~N}_{3}\right), 4.02\left(\mathrm{dd}, 1 \mathrm{H}, J_{1,2} 8.3\right.$, $\left.J_{2,3} 10.8 \mathrm{~Hz}, \mathrm{H}-2^{\mathrm{III}}\right), 4.08$ and $4.21(2 \mathrm{~d}$, each $1 \mathrm{H}$, $\left.\mathrm{OCH}_{2} \mathrm{C}_{6} \mathrm{H}_{5}\right), 4.20\left(\mathrm{~d}, 1 \mathrm{H}, J_{1,2} 8.1 \mathrm{~Hz}, \mathrm{H}-1^{\mathrm{I}}\right), 4.22$ and $4.42\left(2 \mathrm{~d}\right.$, each $\left.1 \mathrm{H}, \mathrm{OCH}_{2} \mathrm{C}_{6} \mathrm{H}_{5}\right), 4.24$ and $4.37(2 \mathrm{~d}$, each $\left.1 \mathrm{H}, \mathrm{OCH}_{2} \mathrm{C}_{6} \mathrm{H}_{5}\right), 4.31\left(\mathrm{~d}, 1 \mathrm{H}, J_{1,2} 7.7 \mathrm{~Hz}, \mathrm{H}-1^{\mathrm{II}}\right)$, 4.64 and $4.81\left(2 \mathrm{~d}\right.$, each $\left.1 \mathrm{H}, \mathrm{OCH}_{2} \mathrm{C}_{6} \mathrm{H}_{5}\right), 4.66$ and 4.87 $\left(2 \mathrm{~d}\right.$, each $\left.1 \mathrm{H}, \mathrm{OCH}_{2} \mathrm{C}_{6} \mathrm{H}_{5}\right), 5.37\left(\mathrm{~d}, 1 \mathrm{H}, \mathrm{H}-1^{\mathrm{III}}\right), 5.52$ $\left(\mathrm{d}, 1 \mathrm{H}, J_{3,4} 3.5, J_{4,5}<1 \mathrm{~Hz}, \mathrm{H}-4^{\mathrm{II}}\right) ;{ }^{13} \mathrm{C}$ NMR $(75.5$ $\left.\mathrm{MHz}, \mathrm{CDCl}_{3}\right): \delta 20.9\left(\mathrm{COCH}_{3}\right), 25.5,26.3,28.6$, and $29.4\left(4 \mathrm{CH}_{2}\right), 51.2\left(\mathrm{CH}_{2} \mathrm{~N}_{3}\right), 56.7\left(\mathrm{C}-2^{\mathrm{III}}\right), 61.0,67.7$, $67.8,69.4,72.9,73.4,74.1,74.7$, and $74.9\left(\mathrm{C}-6^{\mathrm{I}}, \mathrm{C}-6^{\mathrm{II}}, \mathrm{C}-\right.$ $\left.6^{\mathrm{III}}, 5 \mathrm{OCH}_{2} \mathrm{C}_{6} \mathrm{H}_{5}, \mathrm{OCH}_{2}\right), 70.5(2 \mathrm{C}), 70.9,71.9,74.5$, 75.7, 75.8, 78.4, 80.4, 81.5, and $82.5\left(\mathrm{C}-2^{\mathrm{I}}, \mathrm{C}-3^{\mathrm{I}}, \mathrm{C}-4^{\mathrm{I}}, \mathrm{C}-\right.$ $\left.5^{\mathrm{I}}, \mathrm{C}-2^{\mathrm{II}}, \mathrm{C}-3^{\mathrm{II}}, \mathrm{C}-4^{\mathrm{II}}, \mathrm{C}-5^{\mathrm{II}}, \mathrm{C}-3^{\mathrm{III}}, \mathrm{C}-4^{\mathrm{III}}, \mathrm{C}-5^{\mathrm{III}}\right), 99.2$, 101.7 , and $103.4\left(\mathrm{C}-1^{\mathrm{I}}, \mathrm{C}-1^{\mathrm{II}}, \mathrm{C}-1^{\mathrm{III}}\right), 171.1\left(\mathrm{COCH}_{3}\right)$; HRMS of $\mathrm{C}_{69} \mathrm{H}_{78} \mathrm{~N}_{4} \mathrm{O}_{18}(\mathrm{M}, 1250.531):\left[\mathrm{M}+\mathrm{NH}_{4}\right]^{+}$ found 1268.537, calcd 1268.565.

3.16. 6-Azidohexyl (6-O-tert-butyldiphenylsilyl-2-deoxy2-phthalimido- $\beta$-D-glucopyranosyl)-( $1 \rightarrow 3)-(4-O$-acetyl2,6-di- $O$-benzyl- $\beta$-D-galactopyranosyl)-( $1 \rightarrow 4)-2,3,6$-tri$O$-benzyl- $\beta$-D-glucopyranoside (27)

To a soln of $26(0.30 \mathrm{~g}, 0.24 \mathrm{mmol})$ in $\mathrm{CH}_{2} \mathrm{Cl}_{2}(3 \mathrm{~mL})$ and $\mathrm{Py}(0.2 \mathrm{~mL})$ were added 4-dimethylaminopyridine ( $9 \mathrm{mg}, 0.072 \mathrm{mmol}), \mathrm{Et}_{3} \mathrm{~N}(0.2 \mathrm{~mL})$, and tert-butyldiphenylsilyl chloride $(73 \mu \mathrm{L}, 0.29 \mathrm{mmol})$. The mixture was stirred for $20 \mathrm{~h}$, then poured into ice water, extracted with $\mathrm{CH}_{2} \mathrm{Cl}_{2}$, washed with aq satd $\mathrm{NaHCO}_{3}$, dried $\left(\mathrm{MgSO}_{4}\right)$, filtered, and concentrated. Column chromatography (1:1 toluene-EtOAc) of the residue gave 27, isolated as a colourless syrup $(0.30 \mathrm{~g}, 84 \%) ; R_{f}$ $0.42\left(1: 1\right.$ toluene-EtOAc); $[\alpha]_{\mathrm{D}}^{20}+8^{\circ}\left(c 1, \mathrm{CHCl}_{3}\right) ;{ }^{1} \mathrm{H}$ NMR $\left(500 \mathrm{MHz}, \mathrm{CDCl}_{3} ; 2 \mathrm{D}\right.$ TOCSY, ROESY, HSQC): $\delta 1.04\left[\mathrm{~s}, 9 \mathrm{H}, \mathrm{SiC}\left(\mathrm{CH}_{3}\right)_{3}\right], 1.33-1.38(\mathrm{~m}, 4$ $\left.\mathrm{H}, 2 \mathrm{CH}_{2}\right), 1.48-1.55\left(\mathrm{~m}, 2 \mathrm{H}, \mathrm{CH}_{2}\right), 1.58-1.59(\mathrm{~m}, 2 \mathrm{H}$, $\left.\mathrm{CH}_{2}\right), 1.97\left(\mathrm{~s}, 3 \mathrm{H}, \mathrm{COCH}_{3}\right), 3.05\left(\mathrm{~m}, 1 \mathrm{H}, \mathrm{H}-5^{\mathrm{I}}\right), 3.16(\mathrm{t}$, $\left.2 \mathrm{H}, \mathrm{CH}_{2} \mathrm{~N}_{3}\right), 3.51\left(\mathrm{t}, 1 \mathrm{H}, \mathrm{H}-6 \mathrm{~b}^{\mathrm{I}}\right), 3.63\left(\mathrm{dd}, 1 \mathrm{H}, J_{2,3} 9.8\right.$, $\left.J_{3,4} 3.2 \mathrm{~Hz}, \mathrm{H}-3^{\mathrm{II}}\right), 4.04\left(\mathrm{dd}, 1 \mathrm{H}, J_{1,2} 8.3, J_{2,3} 11.0 \mathrm{~Hz}\right.$, $\left.\mathrm{H}-2^{\mathrm{III}}\right), 4.12\left(\mathrm{~d}, 1 \mathrm{H}, \mathrm{OCH}_{\mathrm{HC}} \mathrm{H}_{5}\right), 4.16$ and $4.35(2 \mathrm{~d}$, each $\left.1 \mathrm{H}, \mathrm{OCH}_{2} \mathrm{C}_{6} \mathrm{H}_{5}\right), 4.40\left(\mathrm{~m}, 1 \mathrm{H}, \mathrm{H}-3^{\mathrm{III}}\right), 4.43(\mathrm{~d}, 1$ $\left.\mathrm{H}, \mathrm{OCH} \mathrm{C}_{6} \mathrm{H}_{5}\right), 4.62$ and $4.87(2 \mathrm{~d}$, each $1 \mathrm{H}$, $\left.\mathrm{OCH}_{2} \mathrm{C}_{6} \mathrm{H}_{5}\right), 4.66$ and $4.81\left(2 \mathrm{~d}\right.$, each $\left.1 \mathrm{H}, \mathrm{OCH}_{2} \mathrm{C}_{6} \mathrm{H}_{5}\right)$; ${ }^{13} \mathrm{C} \mathrm{NMR}\left(75.5 \mathrm{MHz}, \mathrm{CDCl}_{3}\right): \delta 19.0\left[\mathrm{SiC}\left(\mathrm{CH}_{3}\right)_{3}\right], 20.5$ $\left(\mathrm{COCH}_{3}\right), 25.5,26.3,28.5$, and $29.4\left(4 \mathrm{CH}_{2}\right), 26.6$ $\left[\mathrm{SiC}\left(\mathrm{CH}_{3}\right)_{3}\right], 51.1\left(\mathrm{CH}_{2} \mathrm{~N}_{3}\right), 56.6\left(\mathrm{C}-2^{\mathrm{III}}\right), 64.8\left(\mathrm{C}-6^{\mathrm{III}}\right)$, $67.7\left(\mathrm{C}-6^{\mathrm{I}}\right), 68.1\left(\mathrm{C}-6^{\mathrm{II}}\right), 69.4\left(\mathrm{OCH}_{2}\right), 70.1\left(\mathrm{C}-4^{\mathrm{II}}\right), 70.9$ $\left(\mathrm{C}-3^{\mathrm{III}}\right), 72.5\left(\mathrm{C}-5^{\mathrm{II}}\right), 73.9\left(\mathrm{C}-4^{\mathrm{III}}\right), 74.5\left(\mathrm{C}-5^{\mathrm{III}}\right), 74.6(\mathrm{C}-$ $\left.5^{\mathrm{I}}\right), 72.9,73.2,74.2,74.7$, and $74.9\left(5 \mathrm{OCH}_{2} \mathrm{C}_{6} \mathrm{H}_{5}\right), 75.5$ $\left(\mathrm{C}-4^{\mathrm{I}}\right), 77.2\left(\mathrm{C}-3^{\mathrm{II}}\right), 79.1\left(\mathrm{C}-2^{\mathrm{II}}\right), 81.4\left(\mathrm{C}-2^{\mathrm{I}}\right), 82.4\left(\mathrm{C}-3^{\mathrm{I}}\right)$, $98.3\left(\mathrm{C}-1^{\mathrm{III}}\right), 101.7\left(\mathrm{C}-1^{\mathrm{II}}\right), 103.3\left(\mathrm{C}-1^{\mathrm{I}}\right), 169.7\left(\mathrm{COCH}_{3}\right)$; HRMS of $\mathrm{C}_{85} \mathrm{H}_{96} \mathrm{~N}_{4} \mathrm{O}_{18}(\mathrm{M}, 1488.648):[\mathrm{M}+\mathrm{H}]^{+}$found 1489.638, calcd 1489.657 .

3.17. 6-Azidohexyl (6-O-tert-butyldiphenylsilyl-2-deoxy3,4-di- $O$ - $p$-methylbenzoyl-2-phthalimido- $\beta$-Dglucopyranosyl)-( $(\rightarrow 3)-(4-O$-acetyl-2,6-di- $O$-benzyl- $\beta$-Dgalactopyranosyl)-( $1 \rightarrow 4)-2,3,6$-tri- $O$-benzyl- $\beta$-Dglucopyranoside (28)

To a soln of $27(0.12 \mathrm{~g}, 0.083 \mathrm{mmol})$ in dry Py $(5 \mathrm{~mL})$ was added dropwise, at $0{ }^{\circ} \mathrm{C}$, a soln of $p$-methylbenzoyl chloride $(27 \mu \mathrm{L}, 0.21 \mathrm{mmol})$ in dry $\mathrm{CH}_{2} \mathrm{Cl}_{2}(1 \mathrm{~mL})$. The mixture was stirred for $18 \mathrm{~h}$ at $\mathrm{rt}$, then poured into ice water, extracted with $\mathrm{CH}_{2} \mathrm{Cl}_{2}$, washed with aq satd $\mathrm{NaHCO}_{3}$, dried $\left(\mathrm{MgSO}_{4}\right)$, filtered, and concentrated. Column chromatography (8:1 toluene-EtOAc) of the residue gave 28 , isolated as a white solid $(0.13 \mathrm{~g}, 91 \%)$; $R_{f} 0.68\left(4: 1\right.$ toluene-EtOAc); $[\alpha]_{\mathrm{D}}^{20}-3^{\circ}\left(c 1, \mathrm{CHCl}_{3}\right)$; ${ }^{1} \mathrm{H}$ NMR $\left(500 \mathrm{MHz}, \mathrm{CDCl}_{3} ; 2 \mathrm{D}\right.$ TOCSY, ROESY, HSQC): $\delta 1.06\left[\mathrm{SiC}\left(\mathrm{CH}_{3}\right)_{3}\right], 1.32-1.36\left(\mathrm{~m}, 4 \mathrm{H}, 2 \mathrm{CH}_{2}\right)$, $1.48-1.51\left(\mathrm{~m}, 2 \mathrm{H}, \mathrm{CH}_{2}\right), 1.58-1.62\left(\mathrm{~m}, 2 \mathrm{H}, \mathrm{CH}_{2}\right), 2.10$ $\left(\mathrm{COCH}_{3}\right), 2.17$ and $2.34\left(2 \mathrm{~s}\right.$, each $\left.3 \mathrm{H}, 2 \mathrm{COC}_{6} \mathrm{H}_{4} \mathrm{CH}_{3}\right)$, $3.13\left(\mathrm{t}, 2 \mathrm{H}, \mathrm{CH}_{2} \mathrm{~N}_{3}\right), 3.16\left(\mathrm{~m}, 1 \mathrm{H}, \mathrm{H}-5^{\mathrm{I}}\right), 3.43(\mathrm{dd}, 1 \mathrm{H}$, $\left.J_{1,2} 8.3, J_{2,3} 9.2 \mathrm{~Hz}, \mathrm{H}-2^{\mathrm{II}}\right), 3.59\left(\mathrm{~m}, 1 \mathrm{H}, \mathrm{H}-5^{\mathrm{II}}\right), 3.63$ $\left(\mathrm{dd}, 1 \mathrm{H}, J_{5,6 \mathrm{~b}} 3.5, J_{6 \mathrm{a}, 6 \mathrm{~b}} 10.8 \mathrm{~Hz}, \mathrm{H}-6 \mathrm{~b}^{\mathrm{I}}\right), 4.18(\mathrm{~d}, 1 \mathrm{H}$, $\left.\mathrm{OC} H \mathrm{HC}_{6} \mathrm{H}_{5}\right), 4.24\left(\mathrm{~d}, 1 \mathrm{H}, \mathrm{OCH} \mathrm{HC}_{6} \mathrm{H}_{5}\right), 4.31(\mathrm{~d}, 1 \mathrm{H}$, $\left.J_{1,2} 8.3 \mathrm{~Hz}, \mathrm{H}-1^{\mathrm{I}}\right), 4.32$ and $4.52(2 \mathrm{~d}$, each $1 \mathrm{H}$, $\left.\mathrm{OCH}_{2} \mathrm{C}_{6} \mathrm{H}_{5}\right), 4.58\left(\mathrm{dd}, 1 \mathrm{H}, J_{1,2} 8.3, J_{2,3} 10.7 \mathrm{~Hz}, \mathrm{H}-\right.$ $\left.2^{\mathrm{III}}\right), 4.73$ and $4.87\left(2 \mathrm{~d}\right.$, each $\left.1 \mathrm{H}, \mathrm{OCH}_{2} \mathrm{C}_{6} \mathrm{H}_{5}\right), 4.69$ and $4.97\left(2 \mathrm{~d}\right.$, each $\left.1 \mathrm{H}, \mathrm{OCH}_{2} \mathrm{C}_{6} \mathrm{H}_{5}\right), 5.81\left(\mathrm{~d}, 1 \mathrm{H}, \mathrm{H}-1^{\mathrm{III}}\right)$, $6.37\left(\mathrm{t}, 1 \mathrm{H}, \mathrm{H}-3{ }^{\mathrm{III}}\right) ;{ }^{13} \mathrm{C}$ NMR $\left(75.5 \mathrm{MHz}, \mathrm{CDCl}_{3}\right): \delta$ $18.7\left[\mathrm{SiC}\left(\mathrm{CH}_{3}\right)_{3}\right], 20.3\left(\mathrm{COCH}_{3}\right), \quad 21.0$ and 21.3 $\left(\mathrm{COC}_{6} \mathrm{H}_{4} \mathrm{CH}_{3}\right)$, 25.3, 26.0, 28.3, and $29.2\left(4 \mathrm{CH}_{2}\right)$, $26.3\left[\mathrm{SiC}\left(\mathrm{CH}_{3}\right)_{3}\right], 50.9\left(\mathrm{CH}_{2} \mathrm{~N}_{3}\right), 55.2\left(\mathrm{C}-2^{\mathrm{III}}\right), 62.6(\mathrm{C}-$ $\left.6^{\mathrm{III}}\right), 67.4\left(\mathrm{C}-6^{\mathrm{I}}\right), 67.9\left(\mathrm{C}-6^{\mathrm{II}}\right), 69.2\left(\mathrm{OCH}_{2}\right), 69.5\left(\mathrm{C}-4^{\mathrm{III}}\right)$, $70.1\left(\mathrm{C}-4^{\mathrm{II}}\right), 70.6\left(\mathrm{C}-3^{\mathrm{III}}\right), 72.5\left(\mathrm{C}-5^{\mathrm{II}}\right), 72.7,73.0,74.1$, 74.4, and $74.5\left(5 \mathrm{OCH}_{2} \mathrm{C}_{6} \mathrm{H}_{5}\right), 74.5\left(\mathrm{C}-5^{\mathrm{I}}\right), 75.2(2 \mathrm{C})(\mathrm{C}-$ $\left.4^{\mathrm{I}}, \mathrm{C}-5^{\mathrm{III}}\right), 77.0\left(\mathrm{C}-3^{\mathrm{II}}\right), 78.9\left(\mathrm{C}-2^{\mathrm{II}}\right), 81.3\left(\mathrm{C}-2^{\mathrm{I}}\right), 82.2(\mathrm{C}-$ $\left.3^{\mathrm{I}}\right), 98.2\left(\mathrm{C}-1^{\mathrm{III}}\right), 101.6\left(\mathrm{C}-1^{\mathrm{II}}\right), 103.1\left(\mathrm{C}-1^{\mathrm{I}}\right), 164.6$ and $165.2\left(2 \mathrm{COC}_{6} \mathrm{H}_{4} \mathrm{CH}_{3}\right), 169.2\left(\mathrm{COCH}_{3}\right)$; HRMS of $\mathrm{C}_{101} \mathrm{H}_{108} \mathrm{~N}_{4} \mathrm{O}_{20} \mathrm{Si} \quad\left(\mathrm{M}\right.$, 1724.736): $[\mathrm{M}+\mathrm{H}]^{+}$found 1725.741, calcd 1725.740 . 
3.18. 6-Azidohexyl (2-deoxy-3,4-di- $O$-p-methylbenzoyl2-phthalimido- $\beta$-D-glucopyranosyl)-( $1 \rightarrow 3)$-(4-O-acetyl2,6-di- $O$-benzyl- $\beta$-D-galactopyranosyl)-( $1 \rightarrow 4)-2,3,6$-tri$O$-benzyl- $\beta$-D-glucopyranoside (29)

A soln of $28(0.10 \mathrm{~g}, 60.2 \mu \mathrm{mol})$ in $1.0 \mathrm{M}$ TBAF in THF $(1 \mathrm{~mL})$ and $\mathrm{AcOH}(1 \mathrm{~mL})(\mathrm{pH} 6)$ was stirred for $1 \mathrm{~h}$ at $0{ }^{\circ} \mathrm{C}$ followed by $4 \mathrm{~h}$ at $\mathrm{rt}$. After the addition of $\mathrm{CH}_{2} \mathrm{Cl}_{2}$, the soln was washed with water and aq satd $\mathrm{NaCl}$, dried $\left(\mathrm{Na}_{2} \mathrm{SO}_{4}\right)$, filtered, and concentrated. Column chromatography (4:1 toluene-EtOAc) of the residue gave 29, isolated as a colourless syrup ( $80 \mathrm{mg}, 89 \%) ; R_{f} 0.34$ (4:1 toluene-EtOAc); $[\alpha]_{\mathrm{D}}^{20}-2^{\circ}\left(c 1, \mathrm{CHCl}_{3}\right) ;{ }^{1} \mathrm{H}$ NMR (500 $\mathrm{MHz}, \mathrm{CDCl}_{3}$; 2D TOCSY, ROESY): $\delta 1.31-1.33$ (m, 4 $\left.\mathrm{H}, 2 \mathrm{CH}_{2}\right), 1.49-1.52\left(\mathrm{~m}, 2 \mathrm{H}, \mathrm{CH}_{2}\right), 1.55-1.59(\mathrm{~m}, 2 \mathrm{H}$, $\left.\mathrm{CH}_{2}\right), 2.14$ (s, $\left.3 \mathrm{H}, \mathrm{COCH}_{3}\right), 2.25$ and $2.33(2 \mathrm{~s}$, each 1 $\left.\mathrm{H}, 2 \mathrm{COC}_{6} \mathrm{H}_{4} \mathrm{CH}_{3}\right), 2.95\left(\mathrm{~m}, 1 \mathrm{H}, \mathrm{H}-5^{\mathrm{I}}\right), 3.18(\mathrm{t}, 2 \mathrm{H}$, $\left.\mathrm{CH}_{2} \mathrm{~N}_{3}\right), 3.55\left(\mathrm{dd}, 1 \mathrm{H}, J_{2,3} 9.6, J_{3,4} 3.5 \mathrm{~Hz}, \mathrm{H}-3^{\mathrm{II}}\right), 3.66$ $\left(\mathrm{dd}, 1 \mathrm{H}, J_{5,6 \mathrm{~b}} 5.5, J_{6 \mathrm{a}, 6 \mathrm{~b}} 10.8 \mathrm{~Hz}, \mathrm{H}-6 \mathrm{~b}^{\mathrm{III}}\right), 3.81(\mathrm{~m}, 1 \mathrm{H}$, $\mathrm{OCH} H), 3.85\left(\mathrm{t}, 1 \mathrm{H}, \mathrm{H}-4^{\mathrm{I}}\right), 3.92\left(\mathrm{~m}, 1 \mathrm{H}, \mathrm{H}-5^{\mathrm{III}}\right), 4.18$ and $4.23\left(2 \mathrm{~d}\right.$, each $\left.1 \mathrm{H}, \mathrm{OCH}_{2} \mathrm{C}_{6} \mathrm{H}_{5}\right), 4.19\left(\mathrm{~d}, 1 \mathrm{H}, J_{1,2}\right.$ $\left.7.7 \mathrm{~Hz}, \mathrm{H}-1^{\mathrm{I}}\right), 4.23$ and $4.45\left(2 \mathrm{~d}\right.$, each $\left.1 \mathrm{H}, \mathrm{OCH}_{2} \mathrm{C}_{6} \mathrm{H}_{5}\right)$, 4.29 and $4.38\left(2 \mathrm{~d}\right.$, each $\left.1 \mathrm{H}, \mathrm{OCH}_{2} \mathrm{C}_{6} \mathrm{H}_{5}\right), 4.34(\mathrm{~d}, 1 \mathrm{H}$, $\left.J_{1,2} 7.7 \mathrm{~Hz}, \mathrm{H}-1^{\mathrm{II}}\right), 4.49\left(\mathrm{dd}, 1 \mathrm{H}, J_{1,2} 8.5, J_{2,3} 10.6 \mathrm{~Hz}\right.$, $\left.\mathrm{H}-2^{\mathrm{III}}\right), 4.66$ and $4.81\left(2 \mathrm{~d}\right.$, each $\left.1 \mathrm{H}, \mathrm{OCH}_{2} \mathrm{C}_{6} \mathrm{H}_{5}\right), 4.67$ and $4.90\left(2 \mathrm{~d}\right.$, each $\left.1 \mathrm{H}, \mathrm{OCH}_{2} \mathrm{C}_{6} \mathrm{H}_{5}\right), 5.49(\mathrm{t}, 1 \mathrm{H}, \mathrm{H}-$ $\left.4^{\mathrm{III}}\right), 5.59\left(\mathrm{~d}, 1 \mathrm{H}, J_{4,5}<1 \mathrm{~Hz}, \mathrm{H}-4^{\mathrm{II}}\right), 5.70(\mathrm{~d}, 1 \mathrm{H}, \mathrm{H}-$ $\left.1^{\mathrm{III}}\right), 6.16\left(\mathrm{dd}, 1 \mathrm{H}, J_{3,4} 9.4 \mathrm{~Hz}, \mathrm{H}-3^{\mathrm{III}}\right) ;{ }^{13} \mathrm{C}$ NMR $(75.5$ $\left.\mathrm{MHz}, \quad \mathrm{CDCl}_{3}\right): \delta 20.3\left(\mathrm{COCH}_{3}\right), 21.0$ and 21.5 $\left(\mathrm{COC}_{6} \mathrm{H}_{4} \mathrm{CH}_{3}\right), 25.6,26.4,28.6$, and $29.4\left(4 \mathrm{CH}_{2}\right)$, $51.2\left(\mathrm{CH}_{2} \mathrm{~N}_{3}\right), 55.0\left(\mathrm{C}-2^{\mathrm{III}}\right), 61.3,67.7,68.0,69.5,72.9$, $73.5,74.2,74.7$, and $74.8 \quad\left(\mathrm{C}-6^{\mathrm{I}}, \mathrm{C}-6^{\mathrm{II}}, \mathrm{C}-6^{\mathrm{III}}, 5\right.$ $\left.\mathrm{OCH}_{2} \mathrm{C}_{6} \mathrm{H}_{5}, \mathrm{OCH}_{2}\right), 69.0,70.1,70.7,72.0,74.5,75.0$, 75.7, 78.2, 81.5, 81.8, and $82.5\left(\mathrm{C}-2^{\mathrm{I}}, \mathrm{C}-3^{\mathrm{I}}, \mathrm{C}-4^{\mathrm{I}}, \mathrm{C}-5^{\mathrm{I}}, \mathrm{C}-\right.$ $\left.2^{\mathrm{II}}, \mathrm{C}-3^{\mathrm{II}}, \mathrm{C}-4^{\mathrm{II}}, \mathrm{C}-5^{\mathrm{II}}, \mathrm{C}-3^{\mathrm{III}}, \mathrm{C}-4^{\mathrm{III}}, \mathrm{C}-5^{\mathrm{III}}\right), 99.3,101.7$, and $103.4\left(\mathrm{C}-1^{\mathrm{I}}, \mathrm{C}-1^{\mathrm{II}}, \mathrm{C}-1^{\mathrm{III}}\right), 165.2$ and 165.5 $\left(\mathrm{COC}_{6} \mathrm{H}_{4} \mathrm{CH}_{3}\right), \quad 171.0 \quad\left(\mathrm{COCH}_{3}\right)$; HRMS of $\mathrm{C}_{85} \mathrm{H}_{90} \mathrm{~N}_{4} \mathrm{O}_{20} \quad(\mathrm{M}, \quad 1486.614):[\mathrm{M}+\mathrm{H}]^{+}$found 1487.621, calcd 1487.623.

\subsection{6-Azidohexyl $(2,3,4,6$-tetra- $O$-acetyl- $\beta$-D- glucopyranosyl)-(1 $\rightarrow 6)-(2-d e o x y-3,4-d i-O-p$ -} methylbenzoyl-2-phthalimido- $\beta$-D-glucopyranosyl)-( $1 \rightarrow$ 3)-(4- $O$-acetyl-2,6-di- $O$-benzyl- $\beta$-D-galactopyranosyl)$(1 \rightarrow 4)$-2,3,6-tri- $O$-benzyl- $\beta$-D-glucopyranoside (30)

(a) A soln of $13(0.27 \mathrm{~g}, 0.26 \mathrm{mmol})$ and $\mathbf{2 4}^{14}(0.23 \mathrm{~g}$, $0.24 \mathrm{mmol})$ in dry $\mathrm{CH}_{2} \mathrm{Cl}_{2}(8 \mathrm{~mL})$, containing $4 \AA$ molecular sieves $(0.2 \mathrm{~g})$, was stirred under $\mathrm{Ar}$ for $0.5 \mathrm{~h}$. After cooling to $-70{ }^{\circ} \mathrm{C}$, TMSOTf $(5 \mu \mathrm{L}, 0.026 \mathrm{mmol})$ was added, and the mixture was stirred for $3 \mathrm{~h}$, during which period the temperature was allowed to reach rt. The mixture was neutralized with $\mathrm{Et}_{3} \mathrm{~N}$, filtered, washed with water, dried $\left(\mathrm{MgSO}_{4}\right)$, filtered, and concentrated. Column chromatography (3:1 toluene-EtOAc) of the residue gave 30, isolated as colourless syrup $(0.20 \mathrm{~g}$, $83 \%)$.

(b) A soln of $\mathbf{9}^{16}(36.5 \mathrm{mg}, 74.2 \mu \mathrm{mol})$ and $29(65 \mathrm{mg}$, $43.6 \mu \mathrm{mol})$ in dry $\mathrm{CH}_{2} \mathrm{Cl}_{2}(1 \mathrm{~mL})$, containing $4 \AA$ molecular sieves $(40 \mathrm{mg})$, was stirred under Ar for $0.5 \mathrm{~h}$. After cooling to $-40{ }^{\circ} \mathrm{C}$, TMSOTf $(1.4 \mu \mathrm{L}, 7.7 \mu \mathrm{mol})$ was added, and the mixture was stirred for $3 \mathrm{~h}$, during which period the temperature was allowed to reach rt. The mixture was neutralized with $\mathrm{Et}_{3} \mathrm{~N}$, filtered, washed with water, dried $\left(\mathrm{MgSO}_{4}\right)$, filtered, and concentrated. Column chromatography (3:1 toluene-EtOAc) of the residue gave 30, isolated as a colourless syrup (46 mg, $58 \%) ; R_{f} 0.56\left(2: 1\right.$ toluene-EtOAc); $[\alpha]_{\mathrm{D}}^{20}+1^{\circ}(c 1$, $\left.\mathrm{CHCl}_{3}\right) ;{ }^{1} \mathrm{H}$ NMR $\left(500 \mathrm{MHz}, \mathrm{CDCl}_{3} ; 2 \mathrm{D}\right.$ TOCSY, ROESY): $\delta 1.34-1.37$ (m, $\left.4 \mathrm{H}, 2 \mathrm{CH}_{2}\right), 1.52-1.55(\mathrm{~m}, 2$ $\left.\mathrm{H}, \mathrm{CH}_{2}\right), 1.60-1.63\left(\mathrm{~m}, 2 \mathrm{H}, \mathrm{CH}_{2}\right), 1.85,1.94,2.00,2.01$, and $2.11\left(5 \mathrm{~s}\right.$, each $\left.3 \mathrm{H}, 5 \mathrm{COCH}_{3}\right), 2.25$ and $2.33(2 \mathrm{~s}$, each $\left.3 \mathrm{H}, 2 \mathrm{COC}_{6} \mathrm{H}_{4} \mathrm{CH}_{3}\right), 3.17\left(\mathrm{~m}, 1 \mathrm{H}, \mathrm{H}-5^{\mathrm{I}}\right), 3.18(\mathrm{t}, 2$ $\left.\mathrm{H}, \mathrm{CH}_{2} \mathrm{~N}_{3}\right), 3.37$ (dd, $\left.1 \mathrm{H}, J_{1,2} 8.1, J_{2,3} 9.7 \mathrm{~Hz}, \mathrm{H}-2^{\mathrm{II}}\right)$, 3.52 (ddd, $\left.1 \mathrm{H}, J_{4,5} 10.1, J_{5,6 \mathrm{a}} 2.4, J_{5,6 \mathrm{~b}} 4.2 \mathrm{~Hz}, \mathrm{H}-5^{\mathrm{IV}}\right)$, 4.09 and $4.38\left(2 \mathrm{~d}\right.$, each $\left.1 \mathrm{H}, \mathrm{OCH}_{2} \mathrm{C}_{6} \mathrm{H}_{5}\right), 4.24$ and 4.35 $\left(2 \mathrm{~d}\right.$, each $\left.1 \mathrm{H}, \mathrm{OCH}_{2} \mathrm{C}_{6} \mathrm{H}_{5}\right), 4.27\left(\mathrm{~d}, 1 \mathrm{H}, J_{1,2} 7.7 \mathrm{~Hz}, \mathrm{H}-\right.$ $\left.1^{\mathrm{I}}\right), 4.28$ and $4.50\left(2 \mathrm{~d}\right.$, each $\left.1 \mathrm{H}, \mathrm{OCH}_{2} \mathrm{C}_{6} \mathrm{H}_{5}\right), 4.64$ and $4.89\left(2 \mathrm{~d}\right.$, each $\left.1 \mathrm{H}, \mathrm{OCH}_{2} \mathrm{C}_{6} \mathrm{H}_{5}\right), 4.68\left(\mathrm{~d}, 1 \mathrm{H}, J_{1,2} 8.1\right.$ $\left.\mathrm{Hz}, \mathrm{H}-1^{\mathrm{IV}}\right), 4.69$ and $4.84\left(2 \mathrm{~d}\right.$, each $\left.1 \mathrm{H}, \mathrm{OCH}_{2} \mathrm{C}_{6} \mathrm{H}_{5}\right)$, $4.94\left(\mathrm{dd}, 1 \mathrm{H}, J_{2,3} 9.9 \mathrm{~Hz}, \mathrm{H}-2^{\mathrm{IV}}\right), 4.98\left(\mathrm{t}, 1 \mathrm{H}, \mathrm{H}-3^{\mathrm{IV}}\right)$, $5.19\left(\mathrm{t}, 1 \mathrm{H}, \mathrm{H}-4^{\mathrm{IV}}\right), 5.40\left(\mathrm{dd}, 1 \mathrm{H}, J_{4,5} 9.9, J_{3,4} 9.2 \mathrm{~Hz}\right.$, $\mathrm{H}-4^{\mathrm{III}}$ ), 5.59 (d, $\left.1 \mathrm{H}, J_{3,4} 3.7, J_{4,5}<1 \mathrm{~Hz}, \mathrm{H}-4^{\mathrm{II}}\right), 5.68(\mathrm{~d}$, $\left.1 \mathrm{H}, J_{1,2} 8.1 \mathrm{~Hz}, \mathrm{H}-1^{\mathrm{III}}\right), 6.28\left(\mathrm{dd}, 1 \mathrm{H}, J_{2,3} 10.8 \mathrm{~Hz}, \mathrm{H}-\right.$ $\left.3^{\mathrm{III}}\right) ;{ }^{13} \mathrm{C} \mathrm{NMR}\left(75.5 \mathrm{MHz}, \mathrm{CDCl}_{3}\right): \delta 20.3,20.4,20.5$, and $20.6(2 \mathrm{C})\left(5 \mathrm{COCH}_{3}\right), 21.4(2 \mathrm{C})\left(2 \mathrm{COC}_{6} \mathrm{H}_{4} \mathrm{CH}_{3}\right)$, 25.6, 26.3, 28.6, and $29.4\left(4 \mathrm{CH}_{2}\right), 51.2\left(\mathrm{CH}_{2} \mathrm{~N}_{3}\right), 55.3$ $\left(\mathrm{C}-2^{\mathrm{III}}\right), 61.4,67.4,67.9,68.2,69.5,72.8,73.2,74.4,74.7$, and $75.1\left(\mathrm{C}-6^{\mathrm{I}}, \mathrm{C}-6^{\mathrm{II}}, \mathrm{C}-6^{\mathrm{III}}, \mathrm{C}-6^{\mathrm{IV}}, 5 \mathrm{OCH}_{2} \mathrm{C}_{6} \mathrm{H}_{5}\right.$, $\left.\mathrm{OCH}_{2}\right), 62.5,67.8,69.7,69.8,70.2,71.4,71.6,72.5$, $74.5,75.0,76.3,77.8,79.0,81.6$, and $82.5\left(\mathrm{C}-2^{\mathrm{I}}, \mathrm{C}-3^{\mathrm{I}}, \mathrm{C}-\right.$ $4^{\mathrm{I}}, \mathrm{C}-5^{\mathrm{I}}, \mathrm{C}-2^{\mathrm{II}}, \mathrm{C}-3^{\mathrm{II}}, \mathrm{C}-4^{\mathrm{II}}, \mathrm{C}-5^{\mathrm{II}}, \mathrm{C}-3^{\mathrm{III}}, \mathrm{C}-4^{\mathrm{III}}, \mathrm{C}-5^{\mathrm{III}}$, $\left.\mathrm{C}-2^{\mathrm{IV}}, \mathrm{C}-3^{\mathrm{IV}}, \mathrm{C}-4^{\mathrm{IV}}, \mathrm{C}-5^{\mathrm{IV}}\right), 98.4,101.0,102.1$, and 103.3 $\left(\mathrm{C}-1^{\mathrm{I}}, \quad \mathrm{C}-1^{\mathrm{II}}, \quad \mathrm{C}-1^{\mathrm{III}}, \quad \mathrm{C}-1^{\mathrm{VV}}\right), \quad 165.1$ and 165.3 $\left(\mathrm{COC}_{6} \mathrm{H}_{4} \mathrm{CH}_{3}\right), \quad 169.0-169.8\left(\mathrm{COCH}_{3}\right) ; \mathrm{HRMS}$ of $\mathrm{C}_{99} \mathrm{H}_{108} \mathrm{~N}_{4} \mathrm{O}_{29} \quad(\mathrm{M}, \quad 1816.709):\left[\mathrm{M}+\mathrm{NH}_{4}\right]^{+}$found 1834.719, calcd 1834.743 .

\subsection{6-Azidohexyl $(2,3,4,6$-tetra- $O$-acetyl- $\beta$-D- glucopyranosyl)-(1 $\rightarrow$ 6)-(2-acetamido-3,4-di- $O$-acetyl-2- deoxy- $\beta$-D-glucopyranosyl)-( $\rightarrow 3)-(4-O$-acetyl-2,6-di- $O$ - benzyl- $\beta$-D-galactopyranosyl)-( $(1 \rightarrow 4)-2,3,6$-tri- $O$-benzyl- $\beta$-D-glucopyranoside (31)}

To a soln of $30(0.20 \mathrm{~g}, 0.11 \mathrm{mmol})$ in $\mathrm{MeOH}(3.5 \mathrm{~mL})$ and $\mathrm{CH}_{2} \mathrm{Cl}_{2}(1.5 \mathrm{~mL})$ was added $\mathrm{NaOMe}(\mathrm{pH} 10)$, and the mixture was stirred for $4 \mathrm{~h}$, then neutralized with Dowex $50 \times 8\left(\mathrm{H}^{+}\right)$, filtered, and concentrated. To a soln of the residue in 1-BuOH $(50 \mathrm{~mL})$ was added 1,2diaminoethane $(10 \mathrm{~mL})$, and the mixture was stirred overnight at $80^{\circ} \mathrm{C}$, then co-concentrated with toluene, 
EtOH, and $\mathrm{CH}_{2} \mathrm{Cl}_{2}$. A soln of the residue in $\mathrm{Py}(30 \mathrm{~mL})$ and $\mathrm{Ac}_{2} \mathrm{O}(30 \mathrm{~mL})$ was stirred for $4 \mathrm{~h}$, then coconcentrated with toluene, EtOH, and $\mathrm{CH}_{2} \mathrm{Cl}_{2}$. Column chromatography (1:1 toluene-EtOAc) of the residue gave 31 , isolated as a colourless glass $(0.16 \mathrm{~g}, 88 \%) ; R_{f}$ $0.45\left(1: 3\right.$ toluene-EtOAc); $[\alpha]_{\mathrm{D}}^{20}-5^{\circ}\left(c 1, \mathrm{CHCl}_{3}\right) ;{ }^{1} \mathrm{H}$ NMR (500 MHz, $\mathrm{CDCl}_{3} ; 2 \mathrm{D}$ TOCSY, ROESY): $\delta$ $1.36-1.38\left(\mathrm{~m}, 4 \mathrm{H}, 2 \mathrm{CH}_{2}\right), 1.52-1.56\left(\mathrm{~m}, 2 \mathrm{H}, \mathrm{CH}_{2}\right)$, 1.63-1.65 (m, $\left.2 \mathrm{H}, \mathrm{CH}_{2}\right), 1.91,1.94,1.95,1.98,2.00$, 2.04, 2.07, and $2.12\left(8 \mathrm{~s}\right.$, each $3 \mathrm{H}, 7 \mathrm{COCH}_{3}$, $\left.\mathrm{NHCOCH}_{3}\right), 3.19$ (t, $\left.2 \mathrm{H}, \mathrm{CH}_{2} \mathrm{~N}_{3}\right), 3.73\left(\mathrm{dd}, 1 \mathrm{H}, J_{5,6 \mathrm{~b}}\right.$ $\left.7.5, J_{6 \mathrm{a}, 6 \mathrm{~b}} 11.6 \mathrm{~Hz}, \mathrm{H}-6 \mathrm{~b}^{\mathrm{III}}\right), 3.95\left(\mathrm{t}, 1 \mathrm{H}, \mathrm{H}-4^{\mathrm{I}}\right), 3.99$ (dd, $\left.1 \mathrm{H}, J_{5,6 \mathrm{a}} 1.8, J_{6 \mathrm{a}, 6 \mathrm{~b}} 12.3 \mathrm{~Hz}, \mathrm{H}-6 \mathrm{a}^{\mathrm{IV}}\right), 4.16\left(\mathrm{dd}, 1 \mathrm{H}, J_{5,6 \mathrm{~b}}\right.$ $\left.4.2 \mathrm{~Hz}, \mathrm{H}-6 \mathrm{~b}^{\mathrm{IV}}\right), 4.27$ and $4.48(2 \mathrm{~d}$, each $1 \mathrm{H}$, $\left.\mathrm{OCH}_{2} \mathrm{C}_{6} \mathrm{H}_{5}\right), 4.34\left(\mathrm{~d}, 1 \mathrm{H}, J_{1,2} 7.7 \mathrm{~Hz}, \mathrm{H}-1^{\mathrm{I}}\right), 4.42$ and $4.55\left(2 \mathrm{~d}\right.$, each $\left.1 \mathrm{H}, \mathrm{OCH}_{2} \mathrm{C}_{6} \mathrm{H}_{5}\right), 4.55\left(\mathrm{~d}, 1 \mathrm{H}, J_{1,2} 7.7\right.$ $\left.\mathrm{Hz}, \mathrm{H}-1^{\mathrm{II}}\right), 4.56\left(\mathrm{~d}, 1 \mathrm{H}, \mathrm{OCH} \mathrm{HC}_{6} \mathrm{H}_{5}\right), 4.63\left(\mathrm{~d}, 1 \mathrm{H}, J_{1,2}\right.$ $\left.7.9 \mathrm{~Hz}, \mathrm{H}-1^{\mathrm{IV}}\right), 5.21\left(\mathrm{t}, 1 \mathrm{H}, \mathrm{H}-3^{\mathrm{IV}}\right), 5.48\left(\mathrm{~d}, 1 \mathrm{H}, J_{3,4} 3.5\right.$, $\left.J_{4,5}<1 \mathrm{~Hz}, \mathrm{H}-4^{\mathrm{II}}\right), 7.14-7.37\left(\mathrm{~m}, 25 \mathrm{H}, 5 \mathrm{OCH}_{2} \mathrm{C}_{6} H_{5}\right)$; ${ }^{13} \mathrm{C}$ NMR $\left(75.5 \mathrm{MHz}, \mathrm{CDCl}_{3}\right): \delta$ 20.3-20.5 $\left(\mathrm{COCH}_{3}\right)$, $22.6\left(\mathrm{NHCOCH}_{3}\right), 25.4,26.2,28.5,29.3\left(4 \mathrm{CH}_{2}\right), 51.0$ $\left(\mathrm{CH}_{2} \mathrm{~N}_{3}\right), 54.1\left(\mathrm{C}-2^{\mathrm{III}}\right), 61.4,67.5,67.9,68.1,69.4,73.0$, $73.5,74.6(2 \mathrm{C})$, and 74.9 (C-6 ${ }^{\mathrm{I}}, \mathrm{C}-6^{\mathrm{II}}, \mathrm{C}-6^{\mathrm{III}}, \mathrm{C}-6^{\mathrm{IV}}, 5$ $\left.\mathrm{OCH}_{2} \mathrm{C}_{6} \mathrm{H}_{5}, \mathrm{OCH}_{2}\right), 67.8,69.3,69.9,71.3,71.4,71.9$, $72.3,72.4,73.5,75.0,76.2,77.4,80.3,81.5$, and $82.2(\mathrm{C}-$ $2^{\mathrm{I}}, \mathrm{C}-3^{\mathrm{I}}, \mathrm{C}-4^{\mathrm{I}}, \mathrm{C}-5^{\mathrm{I}}, \mathrm{C}-2^{\mathrm{II}}, \mathrm{C}-3^{\mathrm{II}}, \mathrm{C}-4^{\mathrm{II}}, \mathrm{C}-5^{\mathrm{II}}, \mathrm{C}-3^{\mathrm{III}}, \mathrm{C}-$ $\left.4^{\mathrm{III}}, \mathrm{C}-5^{\mathrm{III}}, \mathrm{C}-2^{\mathrm{IV}}, \mathrm{C}-3^{\mathrm{IV}}, \mathrm{C}-4^{\mathrm{IV}}, \mathrm{C}-5^{\mathrm{IV}}\right), 100.6,101.2$, 101.8 , and $103.2\left(\right.$ C- $\left.-1^{\mathrm{I}}, \mathrm{C}-1^{\mathrm{II}}, \mathrm{C}-1^{\mathrm{III}}, \mathrm{C}-1^{\mathrm{IV}}\right), 168.9-170.5$ $\left(\mathrm{COCH}_{3}, \mathrm{NHCOCH}_{3}\right)$; HRMS of $\mathrm{C}_{81} \mathrm{H}_{100} \mathrm{~N}_{4} \mathrm{O}_{28}(\mathrm{M}$, 1576.652): $\left[\mathrm{M}+\mathrm{NH}_{4}\right]^{+}$found 1594.647, calcd 1594.686.

3.21. 6-Aminohexyl $\beta$-D-glucopyranosyl- $(1 \rightarrow 6)-2-$ acetamido-2-deoxy- $\beta$-D-glucopyranosyl- $(1 \rightarrow 3)$ - $\beta$-Dgalactopyranosyl-( $1 \rightarrow 4)$ - $\beta$-D-glucopyranoside $(6)$

To a soln of $31(155 \mathrm{mg}, 98.2 \mu \mathrm{mol})$ in $\mathrm{CH}_{2} \mathrm{Cl}_{2}(4 \mathrm{~mL})$ and $\mathrm{MeOH}(2 \mathrm{~mL})$ was added NaOMe (pH 10). The mixture was stirred for $2 \mathrm{~h}$, then neutralized with Dowex $50 \times 8\left(\mathrm{H}^{+}\right)$, filtered, and concentrated, giving crude 32 as a white solid (126 mg). To a soln of 32 in tert $-\mathrm{BuOH}$ $(4 \mathrm{~mL})$ and water $(2 \mathrm{~mL})$ were added $10 \% \mathrm{Pd}-\mathrm{C}(200$ $\mathrm{mg}$ ) and 3 drops of aq $25 \% \mathrm{NH}_{3}$. The mixture was stirred for $3 \mathrm{~h}$ under $\mathrm{H}_{2}$ after which $\mathrm{NH}_{3}$ was removed by bubbling with $\mathrm{N}_{2}$, then $10 \% \mathrm{Pd}-\mathrm{C}(100 \mathrm{mg})$ and 3 drops of $\mathrm{AcOH}$ were added, and the stirring under $\mathrm{H}_{2}$ was continued overnight. The mixture was loaded on a short Dowex $50 \times 8\left(\mathrm{H}^{+}\right)$column, which was first eluted with water to remove contaminations, then with aq $10 \%$ $\mathrm{NH}_{4} \mathrm{OH}$ to give 6, isolated as a white solid after lyophilization (65 mg, 81\%); $R_{f} 0.26$ (2:1:1 AcOH-1$\mathrm{BuOH}-$ water $) ;[\alpha]_{\mathrm{D}}^{20}-2^{\circ}$ (c 1, water); ${ }^{13} \mathrm{C} \mathrm{NMR}(75.5$ $\left.\mathrm{MHz}, \mathrm{D}_{2} \mathrm{O}\right): \delta 23.6\left(\mathrm{NDCOCH}_{3}\right), 26.0,26.7,28.5$, and $29.9\left(4 \mathrm{CH}_{2}\right), 41.0\left(\mathrm{CH}_{2} \mathrm{ND}_{2}\right), 57.1\left(\mathrm{C}-2^{\mathrm{III}}\right), 61.6,62.2$, $62.5,70.2$, and $71.9\left(\mathrm{C}-6^{\mathrm{I}}, \mathrm{C}-6^{\mathrm{II}}, \mathrm{C}-6^{\mathrm{III}}, \mathrm{C}-6^{\mathrm{IV}}, \mathrm{OCH}_{2}\right)$, 69.7, 71.1 (2 C), 71.5, 74.3, 74.6, 75.0, 75.9, 76.1, 76.2, $76.4,77.2,77.4,79.9$, and $83.4\left(\mathrm{C}-2^{\mathrm{I}}, \mathrm{C}-3^{\mathrm{I}}, \mathrm{C}-4^{\mathrm{I}}, \mathrm{C}-5^{\mathrm{I}}, \mathrm{C}-\right.$
$2^{\mathrm{II}}, \mathrm{C}-3^{\mathrm{II}}, \mathrm{C}-4^{\mathrm{II}}, \mathrm{C}-5^{\mathrm{II}}, \mathrm{C}-3^{\mathrm{III}}, \mathrm{C}-4^{\mathrm{III}}, \mathrm{C}-5^{\mathrm{III}}, \mathrm{C}-2^{\mathrm{IV}}, \mathrm{C}-3^{\mathrm{IV}}$, $\left.\mathrm{C}-4^{\mathrm{IV}}, \mathrm{C}-5^{\mathrm{IV}}\right), 103.4,104.2,104.3$, and $104.4\left(\mathrm{C}-1^{\mathrm{I}}, \mathrm{C}-1^{\mathrm{II}}\right.$, $\left.\mathrm{C}-1^{\mathrm{III}}, \mathrm{C}-1^{\mathrm{IV}}\right), \quad 176.3 \quad\left(\mathrm{NDCOCH}_{3}\right)$; HRMS of $\mathrm{C}_{32} \mathrm{H}_{58} \mathrm{~N}_{2} \mathrm{O}_{21}$ (M, 806.353): $[\mathrm{M}+\mathrm{H}]^{+}$found 807.367, calcd 807.361 . For ${ }^{1} \mathrm{H}$ NMR data, see Table 3 .

\subsection{6-Aminohexyl $\beta$-D-glucopyranosyl-( $1 \rightarrow 6$-[ $[\beta$-D- galactopyranosyl- $(1 \rightarrow 4)]$-2-acetamido-2-deoxy- $\beta$-D- glucopyranosyl-( $1 \rightarrow 3)$ - $\beta$-D-galactopyranosyl-( $1 \rightarrow 4)$ - $\beta$-D- glucopyranoside (2)}

To a soln of $6(11.4 \mathrm{mg}, 14.12 \mu \mathrm{mol})$ in aq $50 \mathrm{mM}$ sodium cacodylate buffer pH $7.5(700 \mu \mathrm{L})$, containing 5 $\mathrm{mM} \mathrm{MnCl}_{2}$, BSA (0.5 mg), and $\mathrm{NaN}_{3}(0.02 \%)$, were added alkaline phosphatase (14 U), UDP-galactose (12 $\mathrm{mg}, 19.66 \mu \mathrm{mol}$ ), and $\beta$-1,4-galactosyltransferase (3 U). The mixture was incubated for $20 \mathrm{~h}$ at $37^{\circ} \mathrm{C}$ then water $(100 \mu \mathrm{L})$ was added. UDP-Galactose was removed using a Dowex $1 \times 8\left(\mathrm{Cl}^{-}\right)$column with water as eluent. The eluate was concentrated, and the residue applied to a Bio-Gel P-2 column eluted with aq $0.1 \mathrm{M} \mathrm{NH}_{4} \mathrm{HCO}_{3}$ at a flow rate of $40 \mathrm{~mL} / \mathrm{h}$. The appropriate fractions were freeze-dried to give $2(9.0 \mathrm{mg}, 65 \%)$ and $48(1.0 \mathrm{mg}, 6 \%)$; $R_{f} 0.23(2: 1: 1 \mathrm{AcOH}-1-\mathrm{BuOH}-$ water $) ;[\alpha]_{\mathrm{D}}^{20}-1^{\circ}(c 0.5$, water); HRMS of $\mathrm{C}_{38} \mathrm{H}_{68} \mathrm{~N}_{2} \mathrm{O}_{26}(\mathrm{M}, 968.406)$ : $[\mathrm{M}+\mathrm{H}]^{+}$ found 969.418, calcd 969.414. For ${ }^{1} \mathrm{H} \mathrm{NMR}$ data, see Table 4.

\subsection{3. (3,4,6-Tri- $O$-acetyl-2-deoxy-2-phthalimido- $\beta$-D- glucopyranosyl)-(1 $\rightarrow 3)-(2,4,6$-tri- $O$-acetyl- $\beta$-D- galactopyranosyl)-( $1 \rightarrow 4)-1,2,3,6$-tetra- $O$-acetyl- $\alpha, \beta$-D- glucopyranose (35)}

To a soln of (3,4,6-tri- $O$-acetyl-2-deoxy-2-phthalimido$\beta$-D-glucopyranosyl)-(1 $\rightarrow 3)$-(4- $O$-acetyl-2,6-di- $O$-benzyl- $\beta$-D-galactopyranosyl)-( $1 \rightarrow 4)-1,2,3,6$-tetra- $O$-benzyl- $\beta$-D-glucopyranose $(\mathbf{3 4})^{15}(1.5 \mathrm{~g}, 1.12 \mathrm{mmol})$ in EtOH $(45 \mathrm{~mL})$ and EtOAc $(45 \mathrm{~mL})$ was added $10 \%$ $\mathrm{Pd}-\mathrm{C}(1.78 \mathrm{~g})$ and 2 drops of $\mathrm{AcOH}$, and the mixture was stirred under $\mathrm{H}_{2}$ for $8 \mathrm{~h}$, then filtered over hyflo, and concentrated. Column chromatography $(7: 1$ $\mathrm{CH}_{2} \mathrm{Cl}_{2}-\mathrm{MeOH}$ ) of the residue gave a white solid which was dissolved in $\mathrm{Py}(75 \mathrm{~mL})$ and $\mathrm{Ac}_{2} \mathrm{O}(75 \mathrm{~mL})$, and the soln was stirred overnight, then co-concentrated with toluene, EtOH and $\mathrm{CH}_{2} \mathrm{Cl}_{2}$. Low-pressure column chromatography $(7: 1 \rightarrow 1: 1$ toluene-EtOAc) of the residue gave 35 , isolated as a white foam $(0.95 \mathrm{~g}, 66 \%) ; R_{f}$ 0.45 (1:2 toluene-EtOAc); $[\alpha]_{\mathrm{D}}^{20}-17^{\circ}\left(c 1, \mathrm{CHCl}_{3}\right) ;{ }^{1} \mathrm{H}$ NMR (500 MHz, $\mathrm{CDCl}_{3} ;$ 2D TOCSY, ROESY): $\delta 4.46$ $\left(\mathrm{t}, 1 \mathrm{H}, \mathrm{H}-6 \mathrm{~b}^{\mathrm{III}}\right), 4.80\left(\mathrm{~m}, 1 \mathrm{H}, \mathrm{H}-2^{\mathrm{II}}\right), 4.93(\mathrm{dd}, 0.5 \mathrm{H}$, $\left.J_{1,2} 3.6, J_{2,3} 10.3 \mathrm{~Hz}, \mathrm{H}-2^{\mathrm{I} \alpha}\right), 4.98\left(\mathrm{t}, 0.5 \mathrm{H}, \mathrm{H}-2^{\mathrm{I} \beta}\right), 5.13$ $\left(\mathrm{t}, 0.5 \mathrm{H}, \mathrm{H}-3^{\mathrm{I} \beta}\right), 5.18\left(\mathrm{~m}, 1 \mathrm{H}, \mathrm{H}-4^{\mathrm{III}}\right), 5.63(\mathrm{~d}, 0.5 \mathrm{H}$, $\left.J_{1,2} 8.3 \mathrm{~Hz}, \mathrm{H}-1^{\mathrm{I} \beta}\right), 5.74\left(\mathrm{~m}, 1 \mathrm{H}, \mathrm{H}-3^{\mathrm{III}}\right), 6.19(\mathrm{~d}, 0.5 \mathrm{H}$, $\left.\mathrm{H}-1^{\mathrm{I} \alpha}\right)$, 7.77-7.84 (m, 4 H, Phth); HRMS of $\mathrm{C}_{46} \mathrm{H}_{55} \mathrm{NO}_{27}(\mathrm{M}, 1053.296):\left[\mathrm{M}+\mathrm{NH}_{4}\right]^{+}$found 1071.329, calcd 1071.330. 
3.24. $(3,4,6-T r i-O$-acetyl-2-deoxy-2-phthalimido- $\beta$-Dglucopyranosyl)-( $1 \rightarrow 3)-(2,4,6$-tri- $O$-acetyl- $\beta$-Dgalactopyranosyl)-(1 $\rightarrow 4)-2,3,6$-tri- $O$-acetyl- $\alpha-\mathrm{D}-$ glucopyranosyl trichloroacetimidate (37)

To a soln of 35 (714 mg, $0.68 \mathrm{mmol})$ in dry DMF (10 $\mathrm{mL})$ was added hydrazinium acetate $(67 \mathrm{mg}, 0.73$ mmol). The mixture was stirred for $3 \mathrm{~h}$, then coconcentrated with toluene, EtOH and $\mathrm{CH}_{2} \mathrm{Cl}_{2}$. Lowpressure column chromatography $(3: 1 \rightarrow 1: 1$ tolueneEtOAc) of the residue gave 36, isolated as a white solid (609 $\mathrm{mg}, 89 \%)$. To a soln of $\mathbf{3 6}(519 \mathrm{mg}, 0.51 \mathrm{mmol})$ in dry $\mathrm{CH}_{2} \mathrm{Cl}_{2}(20 \mathrm{~mL})$ was added, at $0{ }^{\circ} \mathrm{C}$, trichloroacetonitrile $(4.9 \mathrm{~mL}, 49 \mathrm{mmol})$ and 1,8-diazabicyclo[5.4.0]undec-7-ene $(0.26 \mathrm{~mL}, 1.25 \mathrm{mmol})$. The mixture was stirred under $\mathrm{Ar}$ for $1.5 \mathrm{~h}$ at $\mathrm{rt}$, then concentrated. Low-pressure column chromatography $(7: 1 \rightarrow 2: 1$ toluene-EtOAc) of the residue gave 37, isolated as a slightly yellow solid $(407 \mathrm{mg}, 69 \%) ; R_{f}$ $0.28\left(1: 2\right.$ toluene-EtOAc); $[\alpha]_{\mathrm{D}}^{20}-8^{\circ}\left(c 1, \mathrm{CHCl}_{3}\right) ;{ }^{1} \mathrm{H}$ NMR (500 MHz, $\mathrm{CDCl}_{3}$; 2D TOCSY, ROESY): $\delta 1.79$, $1.84,1.97,1.99,2.03,2.08,2.10,2.12$, and $2.15(9 \mathrm{~s}$, each $\left.3 \mathrm{H}, 9 \mathrm{COCH}_{3}\right), 3.96\left(\mathrm{~m}, 1 \mathrm{H}, \mathrm{H}-5^{\mathrm{I}}\right), 4.14\left(\mathrm{dd}, 1 \mathrm{H}, J_{1,2}\right.$ $\left.8.3, J_{2,3} 11.0 \mathrm{~Hz}, \mathrm{H}-2^{\mathrm{III}}\right), 4.30\left(\mathrm{~d}, 1 \mathrm{H}, J_{1,2} 8.1 \mathrm{~Hz}, \mathrm{H}-1^{\mathrm{II}}\right)$, $4.36\left(\mathrm{dd}, 1 \mathrm{H}, J_{5,6 \mathrm{a}} 1.8, J_{6 \mathrm{a}, 6 \mathrm{~b}} 12.1 \mathrm{~Hz}, \mathrm{H}-6 \mathrm{a}^{\mathrm{I}}\right), 4.51(\mathrm{dd}, 1$ $\left.\mathrm{H}, J_{5,6 \mathrm{a}} 2.6, J_{6 \mathrm{a}, 6 \mathrm{~b}} 12.3 \mathrm{~Hz}, \mathrm{H}-6 \mathrm{a}^{\mathrm{III}}\right), 4.81\left(\mathrm{dd}, 1 \mathrm{H}, J_{2,3}\right.$ $\left.9.9 \mathrm{~Hz}, \mathrm{H}-2^{\mathrm{II}}\right), 5.00\left(\mathrm{dd}, 1 \mathrm{H}, J_{1,2} 3.5, J_{2,3} 10.1 \mathrm{~Hz}, \mathrm{H}-2^{\mathrm{I}}\right)$, $5.19\left(\mathrm{dd}, 1 \mathrm{H}, J_{3,4} 9.2, J_{4.5} 9.9 \mathrm{~Hz}, \mathrm{H}-4^{\mathrm{III}}\right), 5.41(\mathrm{t}, 1 \mathrm{H}$, $\left.\mathrm{H}-3^{\mathrm{I}}\right), 5.76\left(\mathrm{dd}, 1 \mathrm{H}, \mathrm{H}-3^{\mathrm{III}}\right), 6.43\left(\mathrm{~d}, 1 \mathrm{H}, \mathrm{H}-1^{\mathrm{I}}\right), 7.73-$ $7.75\left(\mathrm{~m}, 4 \mathrm{H}\right.$, Phth), 8.65 (s, $\left.1 \mathrm{H}, \mathrm{OC}(\mathrm{NH}) \mathrm{CCl}_{3}\right) ;{ }^{13} \mathrm{C}$ NMR $\left(75.5 \mathrm{MHz}, \mathrm{CDCl}_{3}\right): \delta 20.3-20.6\left(\mathrm{COCH}_{3}\right), 54.5$ $\left(\mathrm{C}-2^{\mathrm{III}}\right), 60.8,61.5$, and $62.0\left(\mathrm{C}-6^{\mathrm{I}}, \mathrm{C}-6^{\mathrm{II}}, \mathrm{C}-6^{\mathrm{III}}\right), 68.6$, 68.7, 69.2, 69.8 (2 C), 70.0, 70.7, 71.0, 71.8, 74.9, and $75.5\left(\mathrm{C}-2^{\mathrm{I}}, \mathrm{C}-3^{\mathrm{I}}, \mathrm{C}-4^{\mathrm{I}}, \mathrm{C}-5^{\mathrm{I}}, \mathrm{C}-2^{\mathrm{II}}, \mathrm{C}-3^{\mathrm{II}}, \mathrm{C}-4^{\mathrm{II}}, \mathrm{C}-5^{\mathrm{II}}, \mathrm{C}-\right.$ $\left.3^{\mathrm{III}}, \mathrm{C}-4^{\mathrm{III}}, \mathrm{C}-5^{\mathrm{III}}\right), 92.8,97.6$, and $100.6\left(\mathrm{C}-1^{\mathrm{I}}, \mathrm{C}-1^{\mathrm{II}}, \mathrm{C}-\right.$ $\left.1^{\mathrm{III}}\right), 161.8\left(\mathrm{OC}(\mathrm{NH}) \mathrm{CCl}_{3}\right), 168.5-171.6\left(\mathrm{COCH}_{3}\right)$.

\subsection{6-Azidohexyl (3,4,6-tri- $O$-acetyl-2-deoxy-2- phthalimido- $\beta$-D-glucopyranosyl)-( $1 \rightarrow 3)-(2,4,6$-tri- $O$ - acetyl- $\beta$-D-galactopyranosyl)-( $1 \rightarrow 4)-2,3,6$-tri- $O$-acetyl- $\beta$-D-glucopyranoside (38)}

A soln of $37(0.16 \mathrm{~g}, 0.14 \mathrm{mmol})$ and 6-azido-1-hexanol $33(23 \mathrm{mg}, 0.16 \mathrm{mmol})$ in dry $\mathrm{CH}_{2} \mathrm{Cl}_{2}(4 \mathrm{~mL})$, containing $4 \AA$ powdered molecular sieves $(0.1 \mathrm{~g})$, was stirred under Ar for $0.5 \mathrm{~h}$. After cooling to $0{ }^{\circ} \mathrm{C}, \operatorname{AgOTf}(35 \mathrm{mg}, 0.14$ $\mathrm{mmol}$ ) was added, and the mixture was stirred for $1.5 \mathrm{~h}$ at $0{ }^{\circ} \mathrm{C}$, then at $\mathrm{rt}$ for $2 \mathrm{~h}$. After neutralization with $\mathrm{Et}_{3} \mathrm{~N}$, the mixture was filtered over hyflo, washed with water, dried $\left(\mathrm{MgSO}_{4}\right)$, filtered, and concentrated. Column chromatography (1:2 toluene-EtOAc) of the residue gave 38, isolated as a white solid (34 mg, 22\%); $R_{f} 0.41\left(1: 2\right.$ toluene-EtOAc); $[\alpha]_{\mathrm{D}}^{20}+2^{\circ}\left(c 1, \mathrm{CHCl}_{3}\right) ;{ }^{1} \mathrm{H}$ NMR (300 MHz, $\left.\mathrm{CDCl}_{3}\right): \delta 1.26-1.40\left(\mathrm{~m}, 6 \mathrm{H}, 3 \mathrm{CH}_{2}\right)$, 1.52-1.60 (m, $\left.2 \mathrm{H}, \mathrm{CH}_{2}\right), 1.58,1.78,1.84,1.97,1.99$,
$2.03,2.09,2.10$, and $2.15\left(9 \mathrm{~s}\right.$, each $\left.3 \mathrm{H}, 9 \mathrm{COCH}_{3}\right), 3.24$ $\left(\mathrm{t}, 2 \mathrm{H}, \mathrm{CH}_{2} \mathrm{~N}_{3}\right), 3.67\left(\mathrm{t}, 1 \mathrm{H}, \mathrm{H}-4^{\mathrm{I}}\right), 4.09\left(\mathrm{dd}, 1 \mathrm{H}, J_{5,6 \mathrm{~b}}\right.$ $\left.3.5, J_{6 \mathrm{a}, 6 \mathrm{~b}} 12.3 \mathrm{~Hz}, \mathrm{H}-6 \mathrm{~b}^{\mathrm{III}}\right), 4.14$ (dd, $1 \mathrm{H}, J_{1,2} 8.3, J_{2,3}$ $\left.10.9 \mathrm{~Hz}, \mathrm{H}-2^{\mathrm{III}}\right), 4.26\left(\mathrm{~d}, 1 \mathrm{H}, J_{1,2} 8.0 \mathrm{~Hz}, \mathrm{H}-1^{\mathrm{II}}\right), 4.48$ $\left(\mathrm{dd}, 1 \mathrm{H}, J_{5,6 \mathrm{a}} 2.6 \mathrm{~Hz}, \mathrm{H}-6 \mathrm{a}^{\mathrm{III}}\right), 5.06\left(\mathrm{dd}, 1 \mathrm{H}, \mathrm{H}-3^{\mathrm{I}}\right), 5.19$ $\left(\mathrm{dd}, 1 \mathrm{H}, J_{3,4} 9.1 \mathrm{~Hz}, \mathrm{H}-4^{\mathrm{III}}\right), 5.74\left(\mathrm{dd}, 1 \mathrm{H}, \mathrm{H}-3^{\mathrm{III}}\right)$, $7.72-7.75$ and $7.79-7.81(2 \mathrm{~m}$, each $2 \mathrm{H}, \mathrm{Phth}) ;{ }^{13} \mathrm{C}$ NMR (75.5 MHz, $\left.\mathrm{CDCl}_{3}\right): \delta 20.3-20.6\left(\mathrm{COCH}_{3}\right), 25.3$, 26.3 , 28.6, and $29.1\left(4 \mathrm{CH}_{2}\right), 51.2\left(\mathrm{CH}_{2} \mathrm{~N}_{3}\right), 54.5\left(\mathrm{C}-2^{\mathrm{III}}\right)$, $60.8,61.5,62.0$, and $69.7\left(\mathrm{C}-6^{\mathrm{I}}, \mathrm{C}-6^{\mathrm{II}}, \mathrm{C}-6^{\mathrm{III}}, \mathrm{OCH}_{2}\right)$, 68.7, 68.8, 70.0, 70.6, 71.0, 71.5, 71.8, 72.4, 72.6, and 75.5 (2 C) $\left(\mathrm{C}-2^{\mathrm{I}}, \mathrm{C}-3^{\mathrm{I}}, \mathrm{C}-4^{\mathrm{I}}, \mathrm{C}-5^{\mathrm{I}}, \mathrm{C}-2^{\mathrm{II}}, \mathrm{C}-3^{\mathrm{II}}, \mathrm{C}-4^{\mathrm{II}}, \mathrm{C}-\right.$ $\left.5^{\mathrm{II}}, \mathrm{C}-3^{\mathrm{III}}, \mathrm{C}-4^{\mathrm{III}}, \mathrm{C}-5^{\mathrm{III}}\right), 97.6$ and $100.4(2 \mathrm{C})\left(\mathrm{C}-1^{\mathrm{I}}, \mathrm{C}-\right.$ $\left.1^{\mathrm{II}}, \quad \mathrm{C}-1^{\mathrm{III}}\right), \quad 168.5-171.6 \quad\left(\mathrm{COCH}_{3}\right) ;$ HRMS of $\mathrm{C}_{50} \mathrm{H}_{64} \mathrm{~N}_{4} \mathrm{O}_{26} \quad(\mathrm{M}, \quad 1136.380):[\mathrm{M}+\mathrm{Na}]^{+}$found 1159.358, calcd 1159.370.

\subsection{6-Azidohexyl (2-acetamido-3,4,6-tri- $O$-acetyl-2- deoxy- $\beta$-D-glucopyranosyl)-(1 $\rightarrow 3)-(2,4,6$-tri- $O$-acetyl- $\beta$ - D-galactopyranosyl)-( $(\rightarrow 4)-2,3,6$-tri- $O$-acetyl- $\beta$-D- glucopyranoside (39)}

To a soln of $38(22 \mathrm{mg}, 21 \mu \mathrm{mol})$ in $\mathrm{CH}_{2} \mathrm{Cl}_{2}(0.5 \mathrm{~mL})$ and $\mathrm{MeOH}(3 \mathrm{~mL})$ was added $\mathrm{NaOMe}(\mathrm{pH} 10)$, and the mixture was stirred for $2 \mathrm{~h}$, then neutralized with Dowex $50 \times 8\left(\mathrm{H}^{+}\right)$, filtered, and concentrated. To a soln of the residue in $1-\mathrm{BuOH}(10 \mathrm{~mL})$ was added 1,2-diaminoethane $(2 \mathrm{~mL})$, and the mixture was stirred overnight at $80{ }^{\circ} \mathrm{C}$, then co-concentrated with toluene, EtOH and $\mathrm{CH}_{2} \mathrm{Cl}_{2}$. A soln of the residue in $\mathrm{Py}(10 \mathrm{~mL})$ and $\mathrm{Ac}_{2} \mathrm{O}$ $(10 \mathrm{~mL})$ was stirred overnight, then co-concentrated with toluene, $\mathrm{EtOH}$, and $\mathrm{CH}_{2} \mathrm{Cl}_{2}$. Column chromatography (3:1 $\mathrm{CH}_{2} \mathrm{Cl}_{2}$-acetone) of the residue gave 39, isolated as a white solid (17 $\mathrm{mg}, 77 \%) ; R_{f} 0.22(3: 1$ $\mathrm{CH}_{2} \mathrm{Cl}_{2}$-acetone); $[\alpha]_{\mathrm{D}}^{20}+6^{\circ}$ (c $\left.1, \mathrm{CHCl}_{3}\right) ;{ }^{1} \mathrm{H}$ NMR (500 $\mathrm{MHz}, \mathrm{CDCl}_{3} ; 2 \mathrm{D}$ TOCSY, ROESY): $\delta$ 1.25-1.28 (m, $\left.4 \mathrm{H}, 2 \mathrm{CH}_{2}\right), 1.33-1.36\left(\mathrm{~m}, 2 \mathrm{H}, \mathrm{CH}_{2}\right), 1.57-1.59$ $\left(\mathrm{m}, 2 \mathrm{H}, \mathrm{CH}_{2}\right), 1.90,2.00,2.01,2.02,2.08,2.09,2.10$, 2.11, and $2.12\left(9 \mathrm{~s}, 3,3,6,3,3,3,3,3,3 \mathrm{H}, 9 \mathrm{COCH}_{3}\right.$, $\left.\mathrm{NHCOCH}_{3}\right), 3.46(\mathrm{~m}, 1 \mathrm{H}, \mathrm{OCH} \mathrm{H}), 3.59(\mathrm{~m}, 1 \mathrm{H}, \mathrm{H}-$ $\left.5^{\mathrm{I}}\right), 3.66\left(\mathrm{~m}, 1 \mathrm{H}, \mathrm{H}-5^{\mathrm{III}}\right), 4.87\left(\mathrm{dd}, 1 \mathrm{H}, J_{1,2} 8.1, J_{2,3} 9.6\right.$ $\left.\mathrm{Hz}, \mathrm{H}-2^{\mathrm{I}}\right), 5.16\left(\mathrm{t}, 1 \mathrm{H}, \mathrm{H}-3^{\mathrm{I}}\right), 5.32\left(\mathrm{~d}, 1 \mathrm{H}, J_{3,4} 2.4\right.$, $\left.J_{4,5}<1 \mathrm{~Hz}, \mathrm{H}-4^{\mathrm{II}}\right), 5.47\left(\mathrm{t}, 1 \mathrm{H}, \mathrm{H}-3^{\mathrm{III}}\right), 5.60(\mathrm{~d}, 1 \mathrm{H}$, $J_{\mathrm{NH}, 2} 7.5 \mathrm{~Hz}, \mathrm{NHCOCH}$ ); ${ }^{13} \mathrm{C} \mathrm{NMR}(75.5 \mathrm{MHz}$, $\left.\mathrm{CDCl}_{3}\right): \delta$ 20.5-20.8 $\left(\mathrm{NHCOCH}_{3}, \mathrm{COCH}_{3}\right), 25.3$, 26.3, 28.6, and $29.2\left(4 \mathrm{CH}_{2}\right), 51.2\left(\mathrm{CH}_{2} \mathrm{~N}_{3}\right), 56.1(\mathrm{C}-$ $\left.2^{\mathrm{III}}\right), 61.1,61.5,62.1$, and $69.7\left(\mathrm{C}-6^{\mathrm{I}}, \mathrm{C}-6^{\mathrm{II}}, \mathrm{C}-6^{\mathrm{III}}\right.$, $\left.\mathrm{OCH}_{2}\right), 68.7,68.8,71.0,71.1(2 \mathrm{C}), 71.5,71.7,72.5$, 72.7, 75.7, and $75.9\left(\mathrm{C}-2^{\mathrm{I}}, \mathrm{C}-3^{\mathrm{I}}, \mathrm{C}-4^{\mathrm{I}}, \mathrm{C}-5^{\mathrm{I}}, \mathrm{C}-2^{\mathrm{II}}, \mathrm{C}-3^{\mathrm{II}}\right.$, $\left.\mathrm{C}-4^{\mathrm{II}}, \mathrm{C}-5^{\mathrm{II}}, \mathrm{C}-3^{\mathrm{III}}, \mathrm{C}-4^{\mathrm{III}}, \mathrm{C}-5^{\mathrm{III}}\right), 99.5,100.5$, and 100.6 $\left(\mathrm{C}-1^{\mathrm{I}}, \quad \mathrm{C}-1^{\mathrm{II}}, \quad \mathrm{C}-1^{\mathrm{III}}\right), \quad 168.5-171.4 \quad\left(\mathrm{COCH}_{3}\right.$, $\mathrm{NHCOCH}_{3}$ ); HRMS of $\mathrm{C}_{44} \mathrm{H}_{64} \mathrm{~N}_{4} \mathrm{O}_{25}(\mathrm{M}, 1048.386)$ : $[\mathrm{M}+\mathrm{Na}]^{+}$found 1071.397, calcd 1071.375. 
3.27. 6-Azidohexyl 2-acetamido-2-deoxy- $\beta$-Dglucopyranosyl-( $1 \rightarrow 3)$ - $\beta$-D-galactopyranosyl-( $(\rightarrow 4)-\beta$-Dglucopyranoside (40)

To a soln of $39(15 \mathrm{mg}, 14 \mu \mathrm{mol})$ in $\mathrm{CH}_{2} \mathrm{Cl}_{2}(0.5 \mathrm{~mL})$ and $\mathrm{MeOH}(2 \mathrm{~mL})$ was added $\mathrm{NaOMe}(\mathrm{pH} 10)$. The mixture was stirred for $2 \mathrm{~h}$, then neutralized with Dowex $50 \times 8\left(\mathrm{H}^{+}\right)$, filtered, and concentrated. Column chromatography $\left(1: 1 \mathrm{CH}_{2} \mathrm{Cl}_{2}-\mathrm{MeOH}\right)$ of the residue gave 40, isolated as a white solid $(5.3 \mathrm{mg}, 55 \%) ; R_{f} 0.81(1: 2$ $\left.\mathrm{CH}_{2} \mathrm{Cl}_{2}-\mathrm{MeOH}\right) ;[\alpha]_{\mathrm{D}}^{20}-4^{\circ}$ (c 0.5, water); HRMS data of $\mathrm{C}_{26} \mathrm{H}_{46} \mathrm{~N}_{4} \mathrm{O}_{16}$ (M, 670.290): $[\mathrm{M}+\mathrm{H}]^{+}$found 671.300, calcd 671.302. For ${ }^{1} \mathrm{H}$ NMR data, see Table 5.

\subsection{6-Azidohexyl (2-acetamido-3,4,6-tri- $O$-acetyl-2- deoxy- $\beta$-D-glucopyranosyl)-(1 $\rightarrow 3)-(4-O$-acetyl-2,6-di- $O$ - benzyl- $\beta$-D-galactopyranosyl)-( $1 \rightarrow 4)$-2,3,6-tri- $O$-benzyl- $\beta$-D-glucopyranoside (41)}

To a soln of $25(0.21 \mathrm{~g}, 0.15 \mathrm{mmol})$ in $\mathrm{MeOH}(4 \mathrm{~mL})$ and $\mathrm{CH}_{2} \mathrm{Cl}_{2}(2 \mathrm{~mL})$ was added $\mathrm{NaOMe}(\mathrm{pH} 10)$, and the mixture was stirred for $3 \mathrm{~h}$, then neutralized with Dowex $50 \times 8\left(\mathrm{H}^{+}\right)$, filtered, and concentrated. To a soln of the residue in $1-\mathrm{BuOH}(30 \mathrm{~mL})$ was added 1,2-diaminoethane $(6 \mathrm{~mL})$, and the mixture was stirred overnight at $80{ }^{\circ} \mathrm{C}$, then co-concentrated with toluene, $\mathrm{EtOH}$, and $\mathrm{CH}_{2} \mathrm{Cl}_{2}$. A soln of the residue in $\mathrm{Py}(30 \mathrm{~mL})$ and $\mathrm{Ac}_{2} \mathrm{O}$ $(30 \mathrm{~mL})$ was stirred for $48 \mathrm{~h}$, then co-concentrated with toluene, EtOH, and $\mathrm{CH}_{2} \mathrm{Cl}_{2}$. Column chromatography (1:1 toluene-EtOAc) of the residue gave 41, isolated as a colourless glass $(0.15 \mathrm{~g}, 78 \%) ; R_{f} 0.39$ (1:1 tolueneEtOAc); $[\alpha]_{\mathrm{D}}^{20}-2^{\circ}\left(c 1, \mathrm{CHCl}_{3}\right) ;{ }^{1} \mathrm{H}$ NMR $(500 \mathrm{MHz}$, $\mathrm{CDCl}_{3} ; 2 \mathrm{D}$ TOCSY, ROESY): $\delta 1.36-1.42(\mathrm{~m}, 4 \mathrm{H}, 2$ $\left.\mathrm{CH}_{2}\right), 1.51-1.56\left(\mathrm{~m}, 2 \mathrm{H}, \mathrm{CH}_{2}\right), 1.61-1.64(\mathrm{~m}, 2 \mathrm{H}$, $\left.\mathrm{CH}_{2}\right), 1.51,1.94,1.99,2.02$, and $2.05(5 \mathrm{~s}$, each $3 \mathrm{H}, 4$ $\left.\mathrm{COCH}_{3}, \mathrm{NHCOCH}_{3}\right), 3.18\left(\mathrm{t}, 2 \mathrm{H}, \mathrm{CH}_{2} \mathrm{~N}_{3}\right), 3.72$ (dd, 1 $\left.\mathrm{H}, J_{5,6 \mathrm{~b}} 4.1, J_{6 \mathrm{a}, 6 \mathrm{~b}} 11.0 \mathrm{~Hz}, \mathrm{H}-6 \mathrm{~b}^{\mathrm{I}}\right), 3.98\left(\mathrm{t}, 1 \mathrm{H}, \mathrm{H}-4^{\mathrm{I}}\right)$, 4.26 and $4.42\left(2 \mathrm{~d}\right.$, each $\left.1 \mathrm{H}, \mathrm{OCH}_{2} \mathrm{C}_{6} \mathrm{H}_{5}\right), 4.33(\mathrm{~d}, 1 \mathrm{H}$, $\left.J_{1,2} 7.9 \mathrm{~Hz}, \mathrm{H}-1^{\mathrm{I}}\right), 4.38\left(\mathrm{~d}, 1 \mathrm{H}, \mathrm{OCH} \mathrm{HC}_{6} \mathrm{H}_{5}\right), 4.49(\mathrm{~d}, 1$ $\left.\mathrm{H}, J_{1,2} 7.7 \mathrm{~Hz}, \mathrm{H}-1^{\mathrm{II}}\right), 4.72$ and $4.86(2 \mathrm{~d}$, each $1 \mathrm{H}$, $\left.\mathrm{OCH}_{2} \mathrm{C}_{6} \mathrm{H}_{5}\right), 4.74$ and $4.98\left(2 \mathrm{~d}\right.$, each $\left.1 \mathrm{H}, \mathrm{OCH}_{2} \mathrm{C}_{6} \mathrm{H}_{5}\right)$, $5.01\left(\mathrm{t}, 1 \mathrm{H}, \mathrm{H}-4^{\mathrm{III}}\right), 5.09\left(\mathrm{t}, 1 \mathrm{H}, \mathrm{H}-3^{\mathrm{III}}\right), 5.25(\mathrm{~d}, 1 \mathrm{H}$, $\left.J_{2, \mathrm{NH}} 9.2 \mathrm{~Hz}, \mathrm{NH} \mathrm{COCH}_{3}\right), 5.40\left(\mathrm{~d}, 1 \mathrm{H}, J_{3,4} 3.3, J_{4,5}<1\right.$ $\left.\mathrm{Hz}, \mathrm{H}-4^{\mathrm{II}}\right), 7.18-7.36\left(\mathrm{~m}, 25 \mathrm{H}, 5 \mathrm{OCH}_{2} \mathrm{C}_{6} H_{5}\right) ;{ }^{13} \mathrm{C}$ NMR (75.5 MHz, $\left.\mathrm{CDCl}_{3}\right): \delta 20.3-20.5\left(\mathrm{COCH}_{3}\right), 22.5$ $\left(\mathrm{NHCOCH}_{3}\right), 25.4,26.2,28.5$, and $29.3\left(4 \mathrm{CH}_{2}\right), 51.0$ $\left(\mathrm{CH}_{2} \mathrm{~N}_{3}\right), 54.3\left(\mathrm{C}-2^{\mathrm{III}}\right), 61.6,67.9,68.1,69.4,73.0,73.2$, $74.4,74.6$, and 74.8 (C-6 ${ }^{\mathrm{I}}, \mathrm{C}-6^{\mathrm{II}}, \mathrm{C}-6^{\mathrm{III}}, 5 \mathrm{OCH}_{2} \mathrm{C}_{6} \mathrm{H}_{5}$, $\left.\mathrm{OCH}_{2}\right), 68.3,71.4(2 \mathrm{C}), 72.3,72.6,74.7,75.8,78.9,79.5$, 81.4 , and $82.3\left(\mathrm{C}-2^{\mathrm{I}}, \mathrm{C}-3^{\mathrm{I}}, \mathrm{C}-4^{\mathrm{I}}, \mathrm{C}-5^{\mathrm{I}}, \mathrm{C}-2^{\mathrm{II}}, \mathrm{C}-3^{\mathrm{II}}, \mathrm{C}-4^{\mathrm{II}}\right.$, $\left.\mathrm{C}-5^{\mathrm{II}}, \mathrm{C}-3^{\mathrm{III}}, \mathrm{C}-4^{\mathrm{III}}, \mathrm{C}-5^{\mathrm{III}}\right), 100.9,101.7$, and 103.3 (C$\left.1^{\mathrm{I}}, \mathrm{C}-1^{\mathrm{II}}, \mathrm{C}-1^{\mathrm{III}}\right), 168.9,169.4,169.5,170.3$, and 170.4 (4 $\mathrm{COCH}_{3}, \mathrm{NHCOCH}_{3}$ ); HRMS of $\mathrm{C}_{69} \mathrm{H}_{84} \mathrm{~N}_{4} \mathrm{O}_{20}$ (M, 1288.567): $\left[\mathrm{M}+\mathrm{NH}_{4}\right]^{+}$found 1306.629 , calcd 1306.600 .
3.29. 6-Aminohexyl 2-acetamido-2-deoxy- $\beta$-Dglucopyranosyl-( $1 \rightarrow 3)-\beta$-D-galactopyranosyl-(1 $\rightarrow 4)-\beta$-Dglucopyranoside (7)

(a) To a soln of 41 (75 mg, $58.5 \mu \mathrm{mol})$ in $\mathrm{CH}_{2} \mathrm{Cl}_{2}(1 \mathrm{~mL})$ and $\mathrm{MeOH}(2 \mathrm{~mL})$ was added $\mathrm{NaOMe}(\mathrm{pH} 10)$. The mixture was stirred for $1.5 \mathrm{~h}$, then neutralized with Dowex $50 \times 8\left(\mathrm{H}^{+}\right)$, filtered, and concentrated, giving crude 42 as a white solid $(65 \mathrm{mg})$. To a soln of 42 in tert $-\mathrm{BuOH}(15 \mathrm{~mL})$ and water $(10 \mathrm{~mL})$ were added $10 \% \mathrm{Pd}-\mathrm{C}(150 \mathrm{mg})$ and 3 drops of aq $25 \%$ $\mathrm{NH}_{3}$. The mixture was stirred for $3 \mathrm{~h}$ under $\mathrm{H}_{2}$ after which $\mathrm{NH}_{3}$ was removed by bubbling with $\mathrm{N}_{2}$, then $10 \% \mathrm{Pd}-\mathrm{C}(100 \mathrm{mg})$ and 3 drops of $\mathrm{AcOH}$ were added, and the stirring under $\mathrm{H}_{2}$ was continued overnight. The mixture was loaded on a short Dowex $50 \times 8$ $\left(\mathrm{H}^{+}\right)$column, which was first eluted with water to remove contaminations, then with aq $10 \% \mathrm{NH}_{4} \mathrm{OH}$ to give 7, isolated as a white solid after lyophilization (25 mg, 68\%).

(b) To a soln of $\mathbf{4 0}(5.3 \mathrm{mg}, 7.90 \mu \mathrm{mol})$ in tert $-\mathrm{BuOH}$ $(3 \mathrm{~mL})$ and water $(2 \mathrm{~mL})$ was added $10 \% \mathrm{Pd}-\mathrm{C}(40 \mathrm{mg})$ and 2 drops of aq $25 \% \mathrm{NH}_{3}$. The mixture was stirred for $20 \mathrm{~h}$ under $\mathrm{H}_{2}$, after which $\mathrm{NH}_{3}$ was removed by bubbling with $\mathrm{N}_{2}$, then filtered over cotton and concentrated. Chromatography of the residue on a Bio-Gel P-2 column eluted with $0.1 \mathrm{M} \mathrm{NH}_{4} \mathrm{HCO}_{3}$, and subsequent lyophilization yielded 7 , isolated as a white solid (4.2 mg, 83\%); $R_{f} 0.34$ (2:1:1 AcOH-1-BuOH-water); $[\alpha]_{\mathrm{D}}^{20}-2^{\circ}(c 1$, water $) ;{ }^{13} \mathrm{C} \mathrm{NMR}\left(75.5 \mathrm{MHz}, \mathrm{D}_{2} \mathrm{O}\right): \delta$ $23.0\left(\mathrm{NDCOCH}_{3}\right), 25.4,26.1,28.3$, and $29.3\left(4 \mathrm{CH}_{2}\right)$, $40.4\left(\mathrm{CH}_{2} \mathrm{ND}_{2}\right), 56.5\left(\mathrm{C}-2^{\mathrm{III}}\right), 60.9,61.3,61.7$, and 71.2 $\left(\mathrm{C}-6^{\mathrm{I}}, \mathrm{C}-6^{\mathrm{II}}, \mathrm{C}-6^{\mathrm{III}}, \mathrm{OCH}_{2}\right), 69.1,70.5,70.8,73.6,74.4$, $75.3,75.6,75.7,76.5,79.3$, and $82.8\left(\mathrm{C}-2^{\mathrm{I}}, \mathrm{C}-3^{\mathrm{I}}, \mathrm{C}-4^{\mathrm{I}}, \mathrm{C}-\right.$ $\left.5^{\mathrm{I}}, \mathrm{C}-2^{\mathrm{II}}, \mathrm{C}-3^{\mathrm{II}}, \mathrm{C}-4^{\mathrm{II}}, \mathrm{C}-5^{\mathrm{II}}, \mathrm{C}-3^{\mathrm{III}}, \mathrm{C}-4^{\mathrm{III}}, \mathrm{C}-5^{\mathrm{III}}\right), 102.8$, 103.6, and $103.7 \quad\left(\mathrm{C}-1^{\mathrm{I}}, \quad \mathrm{C}-1^{\mathrm{II}}, \quad \mathrm{C}-1^{\mathrm{III}}\right), \quad 175.7$ $\left(\mathrm{NDCOCH}_{3}\right)$; HRMS of $\mathrm{C}_{26} \mathrm{H}_{48} \mathrm{~N}_{2} \mathrm{O}_{16}$ (M, 644.300): $[\mathrm{M}+\mathrm{H}]^{+}$found 645.311, calcd 645.308. For ${ }^{1} \mathrm{H}$ NMR data, see Table 6 .

\subsection{6-Aminohexyl $\beta$-D-galactopyranosyl-(1 $\rightarrow 4)-2$ - acetamido-2-deoxy- $\beta$-D-glucopyranosyl-( $\rightarrow 3)$ - $\beta$-D- galactopyranosyl-( $1 \rightarrow 4)-\beta$-D-glucopyranoside $(3)$}

To a soln of $7(8.2 \mathrm{mg}, 12.71 \mu \mathrm{mol})$ in aq $50 \mathrm{mM}$ sodium cacodylate buffer $\mathrm{pH} 7.5(600 \mu \mathrm{L})$, containing $5 \mathrm{mM}$ $\mathrm{MnCl}_{2}$, BSA (0.5 mg), and $\mathrm{NaN}_{3}(0.02 \%)$, were added alkaline phosphatase (14 U), UDP-galactose $(11.5 \mathrm{mg}$, $18.84 \mu \mathrm{mol})$, and $\beta$-1,4-galactosyltransferase (2.5 U). The mixture was incubated for $20 \mathrm{~h}$ at $37^{\circ} \mathrm{C}$ then water $(200 \mu \mathrm{L})$ was added. UDP-Galactose was removed using a Dowex $1 \times 8\left(\mathrm{Cl}^{-}\right)$column with water as eluent. The eluate was concentrated, and the residue applied to a Bio-Gel P-2 column eluted with aq $0.1 \mathrm{M} \mathrm{NH}_{4} \mathrm{HCO}_{3}$ at a flow rate of $40 \mathrm{~mL} / \mathrm{h}$. The appropriate fractions were 
freeze-dried to give $3(9.6 \mathrm{mg}, 94 \%) ; R_{f} 0.22(2: 1: 1$ $\mathrm{AcOH}-1-\mathrm{BuOH}$-water $) ;[\alpha]_{\mathrm{D}}^{20}-1^{\circ}(c \quad 0.5$, water $)$; HRMS data of $\mathrm{C}_{32} \mathrm{H}_{58} \mathrm{~N}_{2} \mathrm{O}_{21}(\mathrm{M}, 806.353):[\mathrm{M}+\mathrm{H}]^{+}$ found 807.352, calcd 807.361. For ${ }^{1} \mathrm{H}$ NMR data, see Table 7.

\subsection{6-Azidohexyl (3,4,6-tri- $O$-acetyl-2-deoxy-2- phthalimido- $\beta$-D-glucopyranosyl)-( $1 \rightarrow 3)-(2,4,6$-tri- $O$ - acetyl- $\beta$-D-galactopyranosyl)-( $(\rightarrow 4)-(2,3,6$-tri- $O$-acetyl- $\beta$-D-glucopyranosyl)-(1 $\rightarrow$ 6)-2-deoxy-3,4-di- $O$ - $p$ - methylbenzoyl-2-phthalimido- $\beta$-D-glucopyranoside (44)}

To a soln of $37(100 \mathrm{mg}, 87 \mu \mathrm{mol})$ and $\mathbf{4 3}(70 \mathrm{mg}, 104$ $\mu \mathrm{mol})$ in dry $\mathrm{CH}_{2} \mathrm{Cl}_{2}(3 \mathrm{~mL})$, containing $4 \AA$ molecular sieves $(200 \mathrm{mg})$, was added, under Ar at $0{ }^{\circ} \mathrm{C}$, TMSOTf $(2.5 \mu \mathrm{L}, 12.96 \mu \mathrm{mol})$. The mixture was stirred for $1 \mathrm{~h}$ at $0{ }^{\circ} \mathrm{C}$, followed by $1 \mathrm{~h}$ at $\mathrm{rt}$, then neutralized with $\mathrm{Et}_{3} \mathrm{~N}$, filtered over hyflo, washed with water, dried $\left(\mathrm{MgSO}_{4}\right)$, filtered, and concentrated. Low-pressure column chromatography $(4: 1 \rightarrow 2: 1$ toluene-EtOAc) of the residue gave 44, isolated as a white solid (49 mg, 34\%); $R_{f} 0.58$ $\left(1: 2\right.$ toluene-EtOAc); $[\alpha]_{\mathrm{D}}^{20}-11^{\circ}\left(c 1, \mathrm{CHCl}_{3}\right) ;{ }^{1} \mathrm{H}$ NMR $\left(500 \mathrm{MHz}, \mathrm{CDCl}_{3} ;\right.$ 2D TOCSY, ROESY): $\delta$ 1.12-1.20 (m, $\left.4 \mathrm{H}, 2 \mathrm{CH}_{2}\right), 1.25-1.32\left(\mathrm{~m}, 2 \mathrm{H}, \mathrm{CH}_{2}\right)$, $1.49-1.53\left(\mathrm{~m}, 2 \mathrm{H}, \mathrm{CH}_{2}\right), 1.78,1.83,1.96,2.02,2.03$, 2.04, 2.08, 2.10, and $2.14\left(9 \mathrm{~s}\right.$, each $\left.3 \mathrm{H}, 9 \mathrm{COCH}_{3}\right), 2.27$ and $2.33\left(2 \mathrm{~s}\right.$, each $\left.3 \mathrm{H}, 2 \mathrm{COC}_{6} \mathrm{H}_{4} \mathrm{CH}_{3}\right), 3.64(\mathrm{t}, 1 \mathrm{H}, \mathrm{H}-$ $\left.4^{\mathrm{II}}\right), 4.09\left(\mathrm{dd}, 1 \mathrm{H}, J_{5,6 \mathrm{~b}} 3.1, J_{6 \mathrm{a}, 6 \mathrm{~b}} 12.1 \mathrm{~Hz}, \mathrm{H}-6 \mathrm{~b}^{\mathrm{IV}}\right), 4.14$ $\left(\mathrm{dd}, 1 \mathrm{H}, J_{1,2} 8.3, J_{2,3} 10.8 \mathrm{~Hz}, \mathrm{H}-2^{\mathrm{IV}}\right), 4.24\left(\mathrm{~d}, 1 \mathrm{H}, J_{1,2}\right.$ $\left.8.1 \mathrm{~Hz}, \mathrm{H}-1^{1 \mathrm{II}}\right), 4.57\left(\mathrm{~d}, 1 \mathrm{H}, J_{1,2} 7.9 \mathrm{~Hz}, \mathrm{H}-1^{\mathrm{II}}\right), 4.78(\mathrm{dd}$, $\left.1 \mathrm{H}, J_{2,3} 9.9 \mathrm{~Hz}, \mathrm{H}-2^{\mathrm{III}}\right), 4.85\left(\mathrm{dd}, 1 \mathrm{H}, J_{2,3} 9.2 \mathrm{~Hz}, \mathrm{H}-\right.$ $\left.2^{\mathrm{II}}\right), 5.02\left(\mathrm{t}, 1 \mathrm{H}, \mathrm{H}-3^{\mathrm{II}}\right), 5.19\left(\mathrm{t}, 1 \mathrm{H}, \mathrm{H}-4^{\mathrm{IV}}\right), 5.44(\mathrm{~d}, 1$ $\left.\mathrm{H}, J_{1,2} 8.5 \mathrm{~Hz}, \mathrm{H}-1^{\mathrm{I}}\right), 5.75\left(\mathrm{dd}, 1 \mathrm{H}, J_{3,4} 9.2 \mathrm{~Hz}, \mathrm{H}-3^{\mathrm{IV}}\right)$, $6.16\left(\mathrm{dd}, 1 \mathrm{H}, J_{2,3} 10.7, J_{3,4} 9.2 \mathrm{~Hz}, \mathrm{H}-3^{\mathrm{I}}\right) ;{ }^{13} \mathrm{C}$ NMR $\left(75.5 \mathrm{MHz}, \mathrm{CDCl}_{3}\right): \delta 20.2-20.6\left(\mathrm{COCH}_{3}\right), 21.4(2 \mathrm{C})$ $\left(2 \mathrm{COC}_{6} \mathrm{H}_{4} \mathrm{CH}_{3}\right), 25.3,26.0,28.4$, and $28.9\left(4 \mathrm{CH}_{2}\right)$, $51.0\left(\mathrm{CH}_{2} \mathrm{~N}_{3}\right), 54.4$ and $54.8\left(\mathrm{C}-2^{\mathrm{I}}, \mathrm{C}-2^{\mathrm{IV}}\right), 60.8,61.5$, $62.0,68.2$, and $69.6\left(\mathrm{C}-6^{\mathrm{I}}, \mathrm{C}-6^{\mathrm{II}}, \mathrm{C}-6^{\mathrm{III}}, \mathrm{C}-6^{\mathrm{IV}}, \mathrm{OCH}_{2}\right)$, 68.6, 68.7, 69.9, 70.0, 70.5, 70.8, 70.9, 71.3, 71.7, 72.4, 72.6, 74.1, and $75.4(2 \mathrm{C})\left(\mathrm{C}-3^{\mathrm{I}}, \mathrm{C}-4^{\mathrm{I}}, \mathrm{C}-5^{\mathrm{I}}, \mathrm{C}-2^{\mathrm{II}}, \mathrm{C}-3^{\mathrm{II}}\right.$, $\mathrm{C}-4^{\mathrm{II}}, \mathrm{C}-5^{\mathrm{II}}, \mathrm{C}-2^{\mathrm{III}}, \mathrm{C}-3^{\mathrm{III}}, \mathrm{C}-4^{\mathrm{III}}, \mathrm{C}-5^{\mathrm{III}}, \mathrm{C}-3^{\mathrm{IV}}, \mathrm{C}-4^{\mathrm{IV}}, \mathrm{C}-$ $\left.5^{\mathrm{IV}}\right), 97.5,98.0$, and $100.5(2 \mathrm{C})\left(\mathrm{C}-1^{\mathrm{I}}, \mathrm{C}-1^{\mathrm{II}}, \mathrm{C}-1^{\mathrm{III}}, \mathrm{C}-\right.$ $\left.1^{\mathrm{IV}}\right), 165.1$ and $165.4\left(2 \mathrm{COC}_{6} \mathrm{H}_{4} \mathrm{CH}_{3}\right), 168.4-170.6$ $\left(\mathrm{COCH}_{3}\right)$; HRMS of $\mathrm{C}_{68} \mathrm{H}_{81} \mathrm{~N}_{5} \mathrm{O}_{34}(\mathrm{M}, 1151.476)$ : $[\mathrm{M}+$ $\left.\mathrm{NH}_{4}\right]^{+}$found 1169.524, calcd 1169.510 .

\subsection{6-Azidohexyl (2-acetamido-3,4,6-tri- $O$-acetyl-2- deoxy- $\beta$-D-glucopyranosyl)-(1 $\rightarrow 3)-(2,4,6$-tri- $O$-acetyl- $\beta$ - D-galactopyranosyl)-( $1 \rightarrow 4)-(2,3,6$-tri- $O$-acetyl- $\beta$-D- glucopyranosyl)-(1 $\rightarrow 6)$-2-acetamido-3,4-di- $O$-acetyl-2- deoxy- $\beta$-D-glucopyranoside (45)}

To a soln of $44(56 \mathrm{mg}, 63 \mu \mathrm{mol})$ in $\mathrm{CH}_{2} \mathrm{Cl}_{2}(0.45 \mathrm{~mL})$ and $\mathrm{MeOH}(0.55 \mathrm{~mL})$ was added $\mathrm{NaOMe}(\mathrm{pH} 10)$, and the mixture was stirred for $2 \mathrm{~h}$, then neutralized with
Dowex $50 \times 8\left(\mathrm{H}^{+}\right)$, filtered, and concentrated. To a soln of the residue in 1-BuOH $(25 \mathrm{~mL})$ was added 1,2diaminoethane $(5 \mathrm{~mL})$, and the mixture was stirred overnight at $90{ }^{\circ} \mathrm{C}$, then co-concentrated with toluene, EtOH and $\mathrm{CH}_{2} \mathrm{Cl}_{2}$. A soln of the residue in Py $(10 \mathrm{~mL})$ and $\mathrm{Ac}_{2} \mathrm{O}(10 \mathrm{~mL})$ was stirred overnight, then coconcentrated with toluene, EtOH and $\mathrm{CH}_{2} \mathrm{Cl}_{2}$. Column chromatography (3:1 $\mathrm{CH}_{2} \mathrm{Cl}_{2}$-acetone) of the residue gave 45, isolated as a white foam (48 $\mathrm{mg}, 86 \%) ; R_{f} 0.44$ $\left(2: 1 \mathrm{CH}_{2} \mathrm{Cl}_{2}\right.$-acetone); $[\alpha]_{\mathrm{D}}^{20}-10^{\circ}\left(c 1, \mathrm{CHCl}_{3}\right) ;{ }^{1} \mathrm{H}$ NMR (300 MHz, $\left.\mathrm{CDCl}_{3}\right): \delta 1.25-1.34\left(\mathrm{~m}, 4 \mathrm{H}, 2 \mathrm{CH}_{2}\right)$, 1.59-1.64 (m, $4 \mathrm{H}, 2 \mathrm{CH}_{2}$ ) , 1.90, 1.93, 2.00, 2.01, 2.02, $2.08,2.09,2.10,2.11$, and $2.12(10 \mathrm{~s}, 3,3,3,12,3,3,3,3,3,3$ $\left.\mathrm{H}, 11 \mathrm{COCH}_{3}, 2 \mathrm{NHCOCH}_{3}\right), 3.46(\mathrm{~m}, 1 \mathrm{H}, \mathrm{OCH} \mathrm{H})$, $4.12\left(\mathrm{dd}, 1 \mathrm{H}, J_{5,6 \mathrm{~b}} 5.1, J_{6 \mathrm{a}, 6 \mathrm{~b}} 11.7 \mathrm{~Hz}, \mathrm{H}-6 \mathrm{~b}^{\mathrm{II}}\right), 4.44(\mathrm{dd}$, $\left.1 \mathrm{H}, J_{5,6 \mathrm{a}}<1 \mathrm{~Hz}, \mathrm{H}-6 \mathrm{a}^{\mathrm{II}}\right), 4.56\left(\mathrm{~d}, 1 \mathrm{H}, J_{1,2} 7.8 \mathrm{~Hz}, \mathrm{H}-\right.$ $\left.1^{\mathrm{II}}\right), 4.61\left(\mathrm{~d}, 1 \mathrm{H}, J_{1,2} 8.3 \mathrm{~Hz}, \mathrm{H}-1^{\mathrm{I}}\right), 5.12\left(\mathrm{t}, 1 \mathrm{H}, \mathrm{H}-3^{\mathrm{II}}\right)$, $5.24\left(\mathrm{t}, 1 \mathrm{H}, \mathrm{H}-3^{\mathrm{I}}\right), 5.32\left(\mathrm{~d}, 1 \mathrm{H}, J_{3,4} 3.5, J_{4,5}<1 \mathrm{~Hz}, \mathrm{H}-\right.$ $\left.4^{\mathrm{III}}\right) ;{ }^{13} \mathrm{C}$ NMR $\left(75.5 \mathrm{MHz}, \mathrm{CDCl}_{3}\right): \delta \quad 20.5-20.7$ $\left(\mathrm{COCH}_{3}\right), 23.2$ (2 C) (2 $\left.\mathrm{NHCOCH}_{3}\right), 25.4,26.3,28.6$, and $29.2\left(4 \mathrm{CH}_{2}\right), 51.2\left(\mathrm{CH}_{2} \mathrm{~N}_{3}\right), 54.8$ and $56.1\left(\mathrm{C}-2^{\mathrm{I}}, \mathrm{C}-\right.$ $\left.2^{\mathrm{IV}}\right), 61.0,61.5,61.9,68.2$, and $69.4\left(\mathrm{C}-6^{\mathrm{I}}, \mathrm{C}-6^{\mathrm{II}}, \mathrm{C}-6^{\mathrm{III}}\right.$, $\left.\mathrm{C}-6^{\mathrm{IV}}, \mathrm{OCH}_{2}\right), 68.6,68.8,69.2,70.9,71.1$ (2 C), 71.4, 71.7, 72.3, 72.5, 72.8, 73.4, 75.6, and $75.9\left(\mathrm{C}-3^{\mathrm{I}}, \mathrm{C}-4^{\mathrm{I}}, \mathrm{C}-\right.$ $5^{\mathrm{I}}, \mathrm{C}-2^{\mathrm{II}}, \mathrm{C}-3^{\mathrm{II}}, \mathrm{C}-4^{4 \mathrm{II}}, \mathrm{C}-5^{\mathrm{II}}, \mathrm{C}-2^{\mathrm{III}}, \mathrm{C}-3^{\mathrm{III}}, \mathrm{C}-4^{\mathrm{III}}, \mathrm{C}-5^{\mathrm{III}}$, $\left.\mathrm{C}-3^{\mathrm{IV}}, \mathrm{C}-4^{\mathrm{IV}}, \mathrm{C}-5^{\mathrm{IV}}\right), 99.5,100.4(2 \mathrm{C})$, and $100.6\left(\mathrm{C}-1^{\mathrm{I}}\right.$, $\left.\mathrm{C}-1^{\mathrm{II}}, \quad \mathrm{C}-1^{\mathrm{III}}, \quad \mathrm{C}-1^{\mathrm{IV}}\right), \quad 169.0-170.7 \quad\left(\mathrm{COCH}_{3}\right.$, $\mathrm{NHCOCH}_{3}$ ); HRMS of $\mathrm{C}_{56} \mathrm{H}_{81} \mathrm{~N}_{5} \mathrm{O}_{32}$ (M, 1335.486): $\left[\mathrm{M}+\mathrm{NH}_{4}\right]^{+}$found 1353.547, calcd 1353.520.

\subsection{6-Azidohexyl 2-acetamido-2-deoxy- $\beta$-D-}

glucopyranosyl-( $1 \rightarrow 3)$ - $\beta$-D-galactopyranosyl-( $1 \rightarrow 4)$ - $\beta$-Dglucopyranosyl-( $(\rightarrow 6)$-2-acetamido-2-deoxy- $\beta$-Dglucopyranoside (46)

To a soln of $45(48 \mathrm{mg}, 35.9 \mu \mathrm{mol})$ in $\mathrm{CH}_{2} \mathrm{Cl}_{2}(1 \mathrm{~mL})$ and $\mathrm{MeOH}(8 \mathrm{~mL})$ was added $\mathrm{NaOMe}(\mathrm{pH} 10)$. The mixture was stirred for $3 \mathrm{~h}$, then neutralized with Dowex $50 \times 8\left(\mathrm{H}^{+}\right)$, filtered, and concentrated. Column chromatography $\left(1: 2 \mathrm{CH}_{2} \mathrm{Cl}_{2}-\mathrm{MeOH}\right)$ of the residue gave 46, isolated as a white solid ( $25 \mathrm{mg}, 79 \%) ; R_{f} 0.62$ (1:4 $\left.\mathrm{CH}_{2} \mathrm{Cl}_{2}-\mathrm{MeOH}\right) ;[\alpha]_{\mathrm{D}}^{20}-6^{\circ}$ (c 1, water); ${ }^{13} \mathrm{C}$ NMR $\left(75.5 \mathrm{MHz}, \mathrm{D}_{2} \mathrm{O}\right): \delta 22.4(2 \mathrm{C})\left(\mathrm{NDCOCH}_{3}\right), 24.9,25.8$, 28.2 , and $28.7\left(4 \mathrm{CH}_{2}\right), 51.4\left(\mathrm{CH}_{2} \mathrm{~N}_{3}\right), 55.8\left(\mathrm{C}-2^{\mathrm{I}}, \mathrm{C}-2^{\mathrm{IV}}\right)$, $60.3,60.7,61.1,68.8$, and $70.7\left(\mathrm{C}-6^{\mathrm{I}}, \mathrm{C}-6^{\mathrm{II}}, \mathrm{C}-6^{\mathrm{III}}, \mathrm{C}-6^{\mathrm{IV}}\right.$, $\left.\mathrm{OCH}_{2}\right), 68.6,70.0$ (2 C), 70.2, 73.0, 73.8, 73.9, 74.5, 75.1 (3 C), 75.9, 78.7, and 82.2 (C-3 ${ }^{\mathrm{I}}, \mathrm{C}-4^{\mathrm{I}}, \mathrm{C}-5^{\mathrm{I}}, \mathrm{C}-2^{\mathrm{II}}, \mathrm{C}-3^{\mathrm{II}}$, $\mathrm{C}-4^{\mathrm{II}}, \mathrm{C}-5^{\mathrm{II}}, \mathrm{C}-2^{\mathrm{III}}, \mathrm{C}-3^{\mathrm{III}}, \mathrm{C}-4^{\mathrm{III}}, \mathrm{C}-5^{\mathrm{III}}, \mathrm{C}-3^{\mathrm{IV}}, \mathrm{C}-4^{\mathrm{IV}}, \mathrm{C}-$ $\left.5^{\mathrm{IV}}\right), 101.3,102.8,103.0$, and $103.1\left(\mathrm{C}-1^{\mathrm{I}}, \mathrm{C}-1^{\mathrm{II}}, \mathrm{C}-1^{\mathrm{III}}\right.$, $\left.\mathrm{C}-1^{\mathrm{IV}}\right), 174.6$ and 175.1 (2 $\left.\mathrm{NHCOCH}_{3}\right)$; HRMS of $\mathrm{C}_{34} \mathrm{H}_{59} \mathrm{~N}_{5} \mathrm{O}_{21}(\mathrm{M}, 873.370):[\mathrm{M}+\mathrm{Na}]^{+}$found 896.359, calcd 896.360. For ${ }^{1} \mathrm{H}$ NMR data, see Table 8 . 
3.34. 6-Aminohexyl 2-acetamido-2-deoxy- $\beta$-Dglucopyranosyl-( $(\rightarrow 3)-\beta$-D-galactopyranosyl-( $(\rightarrow 4)-\beta$-Dglucopyranosyl- $(1 \rightarrow 6)$-2-acetamido-2-deoxy- $\beta$-Dglucopyranoside (8)

To a soln of $46(10 \mathrm{mg}, 11.44 \mu \mathrm{mol})$ in tert $-\mathrm{BuOH}(2$ $\mathrm{mL})$ and water $(2 \mathrm{~mL})$ was added $10 \% \mathrm{Pd}-\mathrm{C}(50 \mathrm{mg})$ and 1 drop of aq $25 \% \mathrm{NH}_{3}$. The mixture was stirred for $20 \mathrm{~h}$ under $\mathrm{H}_{2}$, then acidified with Dowex $50 \times 8\left(\mathrm{H}^{+}\right)$ ( $\mathrm{pH} 4$ ) and loaded on a short column of Dowex $50 \times 8$ $\left(\mathrm{H}^{+}\right)$. Elution with water, to remove contaminants, followed by $10 \% \mathrm{NH}_{4} \mathrm{OH}$, and subsequent lyophilization yielded 8 , isolated as a white solid $(8 \mathrm{mg}, 82 \%) ; R_{f}$ $0.27(2: 1: 1 \mathrm{AcOH}-1-\mathrm{BuOH}$-water $) ;[\alpha]_{\mathrm{D}}^{20}-2^{\circ}(c 0.5$, water); ${ }^{13} \mathrm{C}$ NMR $\left(75.5 \mathrm{MHz}, \mathrm{D}_{2} \mathrm{O}\right): \delta 22.8(2 \mathrm{C})$ $\left(\mathrm{NDCOCH}_{3}\right), 25.3,25.9,27.4$, and $29.0\left(4 \mathrm{CH}_{2}\right), 40.1$ $\left(\mathrm{CH}_{2} \mathrm{ND}_{2}\right), 56.2$ and $56.3\left(\mathrm{C}-2^{\mathrm{I}}, \mathrm{C}-2^{\mathrm{IV}}\right), 60.7,61.2,61.6$, 69.3 , and $71.2\left(\mathrm{C}-6^{\mathrm{I}}, \mathrm{C}-6^{\mathrm{II}}, \mathrm{C}-6^{\mathrm{III}}, \mathrm{C}-6^{\mathrm{IV}}, \mathrm{OCH}_{2}\right), 69.0$, 70.4 (2 C), 70.7, 73.4, 74.2 (2 C), 75.0, 75.4, 75.6 (2 C), 76.3, 79.1, and $82.6\left(\mathrm{C}-3^{\mathrm{I}}, \mathrm{C}-4^{\mathrm{I}}, \mathrm{C}-5^{\mathrm{I}}, \mathrm{C}-2^{\mathrm{II}}, \mathrm{C}-3^{\mathrm{II}}, \mathrm{C}-4^{\mathrm{II}}\right.$, $\left.\mathrm{C}-5^{\mathrm{II}}, \mathrm{C}-2^{\mathrm{III}}, \mathrm{C}-3^{\mathrm{III}}, \mathrm{C}-4^{\mathrm{III}}, \mathrm{C}-5^{\mathrm{III}}, \mathrm{C}-3^{\mathrm{IV}}, \mathrm{C}-4^{\mathrm{IV}}, \mathrm{C}-5^{\mathrm{IV}}\right)$, $101.9,103.3,103.5$, and $103.6\left(\mathrm{C}-1^{\mathrm{I}}, \mathrm{C}-1^{\mathrm{II}}, \mathrm{C}-1^{\mathrm{III}}, \mathrm{C}-1^{\mathrm{IV}}\right)$, 175.1 and 175.6 (2 $\left.\mathrm{NDCOCH}_{3}\right) ;$ HRMS of $\mathrm{C}_{34} \mathrm{H}_{61} \mathrm{~N}_{3} \mathrm{O}_{21}$ (M, 847.379): $[\mathrm{M}+\mathrm{H}]^{+}$found 848.390, calcd 848.387. For ${ }^{1} \mathrm{H}$ NMR data, see Table 9.

\subsection{6-Aminohexyl $\beta$-D-galactopyranosyl-( $1 \rightarrow 4)$-2- acetamido-2-deoxy- $\beta$-D-glucopyranosyl-( $1 \rightarrow 3)$ - $\beta$-D- galactopyranosyl-( $1 \rightarrow 4)-\beta$-D-glucopyranosyl- $(1 \rightarrow 6)-[\beta$ - D-galactopyranosyl-( $1 \rightarrow 4)$-2-acetamido-2-deoxy- $\beta$-D- glucopyranoside (4)}

To a soln of $8(10.8 \mathrm{mg}, 12.73 \mu \mathrm{mol})$ in aq $50 \mathrm{mM}$ sodium cacodylate buffer $\mathrm{pH} 7.5(600 \mu \mathrm{L})$, containing 5 $\mathrm{mM} \mathrm{MnCl}$, BSA $(0.5 \mathrm{mg})$, and $\mathrm{NaN}_{3}(0.02 \%)$, were added alkaline phosphatase (30 U), UDP-galactose (25 $\mathrm{mg}, 40.96 \mu \mathrm{mol}$ ), and $\beta$-1,4-galactosyltransferase (5 U). The mixture was incubated for $20 \mathrm{~h}$ at $37^{\circ} \mathrm{C}$, then water $(200 \mu \mathrm{L})$ was added. UDP-Galactose was removed using a Dowex $1 \times 8\left(\mathrm{Cl}^{-}\right)$column with water as eluent. The eluate was concentrated, and the residue applied to a Bio-Gel P-2 column eluted with aq $0.1 \mathrm{M} \mathrm{NH}_{4} \mathrm{HCO}_{3}$ at a flow rate of $40 \mathrm{~mL} / \mathrm{h}$. The appropriate fractions were freeze-dried to give $4(11.4 \mathrm{mg}, 76 \%) ; R_{f} 0.15(2: 1: 1$ $\mathrm{AcOH}-1-\mathrm{BuOH}$-water); $[\alpha]_{\mathrm{D}}^{20}-1^{\circ}(c 1$, water $)$; HRMS of $\mathrm{C}_{46} \mathrm{H}_{81} \mathrm{~N}_{3} \mathrm{O}_{31} \quad(\mathrm{M}, 1171.485):[\mathrm{M}+\mathrm{H}]^{+}$found 1172.503, calcd 1172.492. For ${ }^{1}$ H NMR data, see Table 10.

\section{Acknowledgements}

We thank C. Versluis (Utrecht University) for recording the HRMS spectra, and N. Matsouki for the synthesis of building blocks 13 and 20. This work was financially supported by the EU, project BIO CT 95-0138.

\section{References}

1. Centers for Disease Control and Prevention. Prevention of pneumococcal disease: recommendations of the Advisory Committee on Immunization Practices (ACIP), $M M W R$ Morb. Mortal. Wkly. Rep. 1997, 46, 1-24.

2. Fine, M. J.; Smith, M. A.; Carson, C. A.; Mutha, S. S.; Sankey, S. S.; Weissfeld, L. A.; Kapoor, W. N. J. Am. Med. Assoc. 1996, 275, 134-141.

3. Doern, G. V.; Brueggemann, A. B.; Huynh, H.; Wingert, E.; Rhomberg, P. Emerg. Infect. Dis. 1999, 5, 757-765.

4. Klugman, K. P. Clin. Microbiol. Rev. 1990, 3, 171-196.

5. Robbins, J. B.; Austrian, R.; Lee, C. J.; Rastogi, S. C.; Schiffman, G.; Henrichsen, J.; Mäkalä, P. H.; Broome, C. V.; Facklam, R. R.; Tiesjema, R. H.; Parke, J. C., Jr. J. Infect. Dis. 1983, 148, 1136-1159.

6. Örtqvist, A.; Hedlund, J.; Burman, L.-Å.; Elbel, E.; Höfer, M.; Leinonen, M.; Lindblad, I.; Sundelöf, B.; Kalin, M. Lancet 1998, 351, 399-403.

7. Rennels, M. B.; Edwards, K. M.; Keyserling, H. L.; Reisinger, K. S.; Hogerman, D. A.; Madore, D. V.; Chang, I.; Paradiso, P. R.; Malinoski, F. J.; Kimura, A. Pediatrics 1998, 101, 604-611.

8. Adams, W. G.; Deaver, K. A.; Cochi, S. L. J. Am. Med. Assoc. 1993, 269, 221-226.

9. Richmond, P.; Borrow, R.; Miller, E.; Clark, S.; Sadler, F.; Fox, A.; Begg, N.; Morris, R.; Cartwright, K. J. Infect. Dis. 1999, 179, 1569-1572.

10. Lindberg, B.; Lönngren, J.; Powell, D. A. Carbohydr. Res. 1977, 58, 177-186.

11. Niggemann, J.; Kamerling, J. P.; Vliegenthart, J. F. G. Bioorg. Med. Chem. 1998, 6, 1605-1612.

12. Niggemann, J.; Kamerling, J. P.; Vliegenthart, J. F. G. J. Chem. Soc. Perkin Trans. 1 1998, 3011-3020.

13. Mawas, F.; Niggemann, J.; Jones, C.; Corbel, M. J.; Kamerling, J. P.; Vliegenthart, J. F. G. Infect. Immun. 2002, 70, 5107-5114.

14. Michalik, D.; Vliegenthart, J. F. G.; Kamerling, J. P. J. Chem. Soc. Perkin Trans. 1 2002, 1973-1981.

15. Joosten, J. A. F.; Kamerling, J. P.; Vliegenthart, J. F. G. Carbohydr. Res. (People BAV020 same issue).

16. Schmidt, R. R.; Stumpp, M. Liebings Ann. Chem. 1983, $1249-1256$.

17. Grundler, G.; Schmidt, R. R. Carbohydr. Res. 1985, 135 , 203-218.

18. Hill, R. L.; Brew, K. Adv. Enzymol. Rel. Areas Mol. Biol. 1975, 43, 411-489.

19. Kamerling, J. P. In Streptococcus pneumoniae, Molecular Biology \& Mechanisms of Disease; Thomasz, A., Ed. Pneumococcal polysaccharides: a chemical view; Mary Ann Liebert: New York, 2000; pp 81-114. 